ESAIM: M2AN 49 (2015) 421-458

DOI: $10.1051 / \mathrm{m} 2 \mathrm{an} / 2014039$
ESAIM: Mathematical Modelling and Numerical Analysis

www.esaim-m2an.org

\title{
ON THE STABLE NUMERICAL APPROXIMATION OF TWO-PHASE FLOW WITH INSOLUBLE SURFACTANT
}

\author{
John W. Barrett ${ }^{1}$, Harald Garcke ${ }^{2}$ and Robert NÜRnBerg ${ }^{1}$
}

\begin{abstract}
We present a parametric finite element approximation of two-phase flow with insoluble surfactant. This free boundary problem is given by the Navier-Stokes equations for the two-phase flow in the bulk, which are coupled to the transport equation for the insoluble surfactant on the interface that separates the two phases. We combine the evolving surface finite element method with an approach previously introduced by the authors for two-phase Navier-Stokes flow, which maintains good mesh properties. The derived finite element approximation of two-phase flow with insoluble surfactant can be shown to be stable. Several numerical simulations demonstrate the practicality of our numerical method.
\end{abstract}

Mathematics Subject Classification. 35Q35, 65M12, 76D05, 76D27, 76M10.

Received November 18, 2013. Revised June 25, 2014.

Published online February 19, 2015.

\section{INTRODUCTION}

The presence of surface active agents (surfactants) has a noticeable effect on the deformation of fluid-fluid interfaces, because these impurities lower the surface tension. In addition, surfactant gradients along the fluidfluid interface cause tangential stresses leading to fluid motion (the Marangoni effect). As a result, the presence of surfactants can have a dramatic effect on droplet shapes during their evolution. Surfactants are applied in a wide range of technologies to increase the efficiency of wetting agents, detergents, foams and emulsion stabilisers.

In this paper we study the effect of an insoluble surfactant in a two-phase flow. The mathematical model consists of the Navier-Stokes equations in the two phases, together with jump conditions at the free boundary separating the two phases. In particular, the Laplace-Young condition has to hold, which is a force balance involving forces resulting from the two fluids. These forces are expressed with the help of the stress tensor as well as surface tension forces and tangential Marangoni forces, where the latter two involve the surfactant concentration. The insoluble surfactant is transported on the interface by advection and possibly by diffusion. The overall system is quite complex, as a free boundary problem for the Navier-Stokes equations and an advection-diffusion equation on the evolving interface have to be solved simultaneously.

The mathematical analysis for the two-phase fluid flow problem with surfactants is still in its early stages. We refer to [26], where a dissipation inequality for free surface flow with an insoluble surfactant was derived,

Keywords and phrases. Incompressible two-phase flow, insoluble surfactants, finite elements, front tracking, ALE ESFEM.

1 Department of Mathematics, Imperial College, London, SW7 2AZ, UK. j.barrett@imperial.ac.uk;

robert.nurnberg@imperial.ac.uk

2 Fakultät für Mathematik, Universität Regensburg, 93040 Regensburg, Germany. harald.garcke@ur.de 
and to [12-14], where well-posedness and stability of equilibria for two-phase flows with soluble surfactants were shown. In particular, in [13] an energy inequality was crucial in order to study the stability of equilibria. Although these two-phase results are for soluble surfactants, the techniques should be applicable to show local existence of strong solutions for the case of insoluble surfactants. To the best of the authors' knowledge no existence and uniqueness results for two-phase flow with insoluble surfactants are available in the literature at present. In this paper, it is our aim to develop a numerical method that fulfills a discrete variant of this energy inequality and, in addition, conserves the surfactant mass and the volume of the two phases. Here we note that many of the existing numerical methods for two-phase flow with insoluble surfactant may lose mass of one of the fluid phases, or may face stability issues. In fact, to our knowledge, the numerical method presented in this paper is the first approximation of two-phase flow with insoluble surfactant in the literature that can be shown to satisfy a discrete energy law.

Different interface capturing and interface tracking methods have been used to numerically compute twophase flows with (in-)soluble surfactants. Popular such approaches are volume of fluid methods, $[1,17,30,35]$; level set methods, [28, 38, 40, 41]; front tracking methods, [31-33, 39] and arbitrary Lagrangian-Eulerian methods, $[24,34,42]$. Another approach to model and numerically simulate two-phase fluids involving surfactants involves diffuse interface approaches and we refer to [21,25,37] and [23] for details.

In this work we use parametric finite elements to describe the fluid-fluid interface with an unfitted coupling to the fluid flow in the bulk, which is also discretized with the help of finite elements. Unfitted in this context means that the mesh points used to describe the interface are not, in general, mesh points of the underlying bulk finite element mesh. Our approach is based on earlier work by the authors on two-phase flow for incompressible Stokes and Navier-Stokes flow involving surface tension effects, see [3,9] for details. As mentioned above, apart from capturing the interface in a two-phase flow, one also has to accurately capture the advection and diffusion of the surfactant on the interface. Here we make use of a variant of the evolving surface finite element method (ESFEM) introduced in $[19,20]$. In order to accurately discretize the advection-diffusion equation on the evolving interface, it is important to evolve the grid points representing the interface in such a way, that the mesh does not degenerate. In particular, it is important to avoid the coalescence of vertices or a velocity induced coarsening at parts of the interface, see e.g. Figures 2 and 3 in Section 5. It turns out that moving vertices with the fluid velocity or with the normal part of the fluid velocity typically leads to mesh degeneracies. Hence in this paper we follow the approach from [3] and allow the grid points to have a tangential velocity that is independent of the surrounding fluid motion. We note that the idea to allow for an implicit, nonzero discrete tangential velocity goes back to earlier work by the present authors, who introduced novel numerical methods with excellent mesh properties for curvature driven flows and moving boundary problems in e.g. [6-8]. In fact, we are able to show that our semidiscrete continuous-in-time finite element approximations lead to equidistributed mesh points on the interface in two space dimensions, and to conformal polyhedral surfaces, which also have good mesh properties, in three space dimensions. Using this approach also ensures that, due to the good mesh properties, the surface partial differential equation for the insoluble surfactant can be solved accurately.

An important issue in surface tension driven flows is to compute curvature quantities with the help of the chosen interface representation. Our approach uses a parametric approximation of the interface, and hence we use a variant of an idea by Dziuk to compute the mean curvature. In fact, in [18] the identity

$$
\Delta_{s} \overrightarrow{\mathrm{id}}=\vec{\varkappa},
$$

is used in a discrete setting to compute an approximation of the mean curvature. Here $\Delta_{s}$ is the LaplaceBeltrami operator, $\vec{\varkappa}$ is the mean curvature vector and id denotes the identity function in $\mathbb{R}^{d}$. This idea was used in [2] for an approximation of free capillary flows, and in [11] for two-phase flows. A discretization of a variant of (1.1) was used by the present authors in [3,9] to derive approximations of two-phase flow with better mesh properties. As mentioned above, this approach leads to tangential motion of the mesh points on the interface that is independent of the fluid motion. This has to be taken into account when solving the advectiondiffusion equation on the interface, and in our case we naturally obtain the so-called arbitrary Lagrangian Eulerian evolving surface finite element method (ALE ESFEM), see [22]. 
The structure of this article is as follows. In the next section we first state the mathematical formulation of the problem and discuss the relevant conserved quantities and an energy identity. In addition, different weak formulations are introduced, which form the basis for the semidiscrete (continuous-in-time) finite element approximations in Section 3 and their fully discrete counterparts in Section 4. In each case we state finite element approximations based on two different approaches for the approximation of curvature. The first method uses the curvature discretization from [18], and we denote the resultant semidiscrete scheme by $\left(\mathrm{A}_{\mathrm{sd}}\right)$, see Section 3.1. The second method uses the curvature discretization introduced by the present authors in $[3,6,7]$, and we denote this semidiscrete scheme by $\left(\mathrm{B}_{\mathrm{sd}}\right)$, see Section 3.2. The corresponding fully discrete finite element schemes are denoted by $\left(\mathrm{A}_{\mathrm{fd}}\right)$ and $\left(\mathrm{B}_{\mathrm{fd}}\right)$, see Section 4.1 and Section 4.2, respectively. Let us now state the main features of the different finite element approximations.

(a) Stability and energy bounds.

The semidiscrete methods $\left(\mathrm{A}_{\mathrm{sd}}\right)$ and $\left(\mathrm{B}_{\mathrm{sd}}\right)$ fulfill an energy inequality in two space dimensions.

(b) Mass (volume) conservation of the phases.

An XFEM variant of $\left(\mathrm{B}_{\mathrm{sd}}\right)$, which only uses one additional basis function, leads to exact mass (volume) conservation of the two phases.

(c) Conservation of the total amount of surfactant concentration.

The semidiscretizations $\left(A_{s d}\right),\left(B_{s d}\right)$ and the fully discrete methods $\left(A_{f d}\right),\left(B_{f d}\right)$ all conserve the total surfactant concentration.

(d) Discrete maximum principle for the surfactant concentration.

The semidiscrete approximation $\left(\mathrm{A}_{\mathrm{sd}}\right)$ allows for a discrete maximum principle, i.e. the surfactant concentration remains nonnegative if the initial concentration has this property. A weaker lower bound can be shown for the surfactant concentration of the scheme $\left(\mathrm{B}_{\mathrm{sd}}\right)$, see Remark 3.7 below.

(e) Interface mesh quality.

For the semidiscrete version $\left(\mathrm{B}_{\mathrm{sd}}\right)$ good mesh properties can be shown, i.e., in particular, in two space dimensions for each time the distance between consecutive mesh points is constant. In practice this leads to very good mesh properties also for the fully discrete method $\left(\mathrm{B}_{\mathrm{fd}}\right)$. The discretizations $\left(\mathrm{A}_{\mathrm{sd}}\right),\left(\mathrm{A}_{\mathrm{fd}}\right)$ can lead to a poor mesh quality resulting, for example, in the coalescence of grid points.

(f) Existence and uniqueness.

For the fully discrete approximations $\left(\mathrm{A}_{\mathrm{fd}}\right),\left(\mathrm{B}_{\mathrm{fd}}\right)$ existence and uniqueness can be shown. In contrast, no existence and uniqueness results are known for the semidiscrete versions $\left(A_{\mathrm{sd}}\right)$ and $\left(\mathrm{B}_{\mathrm{sd}}\right)$.

In Section 5 we present several numerical simulations for $\left(\mathrm{A}_{\mathrm{fd}}\right)$ and $\left(\mathrm{B}_{\mathrm{fd}}\right)$, in both two and three space dimensions, which in particular show the effect of surfactants on the interface evolution. Taking the above list of properties and the numerical simulations presented into account, we prefer the method $\left(\mathrm{B}_{\mathrm{fd}}\right)$ in practice.

\section{Mathematical Formulation}

\subsection{Governing equations}

Let $\Omega \subset \mathbb{R}^{d}$ be a given domain, where $d=2$ or $d=3$. We now seek a time dependent interface $(\Gamma(t))_{t \in[0, T]}$, $\Gamma(t) \subset \Omega$, which for all $t \in[0, T]$ separates $\Omega$ into a domain $\Omega_{+}(t)$, occupied by one phase, and a domain $\Omega_{-}(t):=\Omega \backslash \overline{\Omega_{+}(t)}$, which is occupied by the other phase. Here the phases could represent two different liquids, or a liquid and a gas. Common examples are oil/water or water/air interfaces. See Figure 1 for an illustration. For later use, we assume that $(\Gamma(t))_{t \in[0, T]}$ is a sufficiently smooth evolving hypersurface without boundary that is parameterized by $\vec{x}(\cdot, t): \Upsilon \rightarrow \mathbb{R}^{d}$, where $\Upsilon \subset \mathbb{R}^{d}$ is a given reference manifold, i.e. $\Gamma(t)=\vec{x}(\Upsilon, t)$. Then

$$
\overrightarrow{\mathcal{V}}(\vec{z}, t):=\vec{x}_{t}(\vec{q}, t) \quad \forall \vec{z}=\vec{x}(\vec{q}, t) \in \Gamma(t)
$$




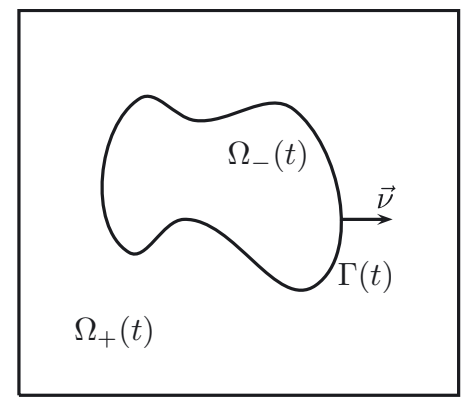

Figure 1 . The domain $\Omega$ in the case $d=2$.

defines the velocity of $\Gamma(t)$, and $\overrightarrow{\mathcal{V}}$. $\vec{\nu}$ is the normal velocity of the evolving hypersurface $\Gamma(t)$, where $\vec{\nu}(t)$ is the unit normal on $\Gamma(t)$ pointing into $\Omega_{+}(t)$. Moreover, we define the space-time surface

$$
\mathcal{G}_{T}:=\bigcup_{t \in[0, T]} \Gamma(t) \times\{t\}
$$

Let $\rho(t)=\rho_{+} \mathcal{X}_{\Omega_{+}(t)}+\rho_{-} \mathcal{X}_{\Omega_{-}(t)}$, with $\rho_{ \pm} \in \mathbb{R}_{>0}$, denote the fluid densities, where here and throughout $\mathcal{X}_{\mathcal{A}}$ defines the characteristic function for a set $\mathcal{A}$. Denoting by $\vec{u}: \Omega \times[0, T] \rightarrow \mathbb{R}^{d}$ the fluid velocity, by $\underline{\underline{\sigma}}: \Omega \times[0, T] \rightarrow \mathbb{R}^{d \times d}$ the stress tensor, and by $\vec{f}: \Omega \times[0, T] \rightarrow \mathbb{R}^{d}$ a possible forcing, the incompressible $\overline{\bar{N}}$ avier-Stokes equations in the two phases are given by

$$
\begin{array}{rlrl}
\rho\left(\overrightarrow{u_{t}}+(\vec{u} . \nabla) \vec{u}\right)-\nabla \cdot \underline{\underline{\sigma}} & =\vec{f}:=\rho \overrightarrow{f_{1}}+\overrightarrow{f_{2}} & & \text { in } \Omega_{ \pm}(t), \\
\nabla \cdot \vec{u} & =0 & & \text { in } \Omega_{ \pm}(t), \\
\vec{u} & =\overrightarrow{0} & & \text { on } \partial_{1} \Omega, \\
\vec{u} \cdot \overrightarrow{\mathrm{n}}=0, \quad \underline{\underline{\sigma}} \overrightarrow{\mathrm{n}} \cdot \overrightarrow{\mathrm{t}}=0 \quad \forall \overrightarrow{\mathrm{t}} \in\{\overrightarrow{\mathrm{n}}\}^{\perp} & & \text { on } \partial_{2} \Omega,
\end{array}
$$

where $\partial \Omega=\partial_{1} \Omega \cup \partial_{2} \Omega$, with $\partial_{1} \Omega \cap \partial_{2} \Omega=\emptyset$, denotes the boundary of $\Omega$ with outer unit normal $\overrightarrow{\mathrm{n}}$ and $\{\overrightarrow{\mathrm{n}}\}^{\perp}:=\left\{\overrightarrow{\mathrm{t}} \in \mathbb{R}^{d}: \overrightarrow{\mathrm{t}} \cdot \overrightarrow{\mathrm{n}}=0\right\}$. Hence (2.3c) prescribes a no-slip condition on $\partial_{1} \Omega$, while (2.3d) prescribes a free-slip condition on $\partial_{2} \Omega$. In addition, the stress tensor in (2.3a) is defined by

$$
\underline{\underline{\sigma}}=\mu\left(\nabla \vec{u}+(\nabla \vec{u})^{T}\right)-p \underline{\underline{I d}}=2 \mu \underline{\underline{D}}(\vec{u})-p \underline{\underline{I d}},
$$

where $\underline{\underline{I d}} \in \mathbb{R}^{d \times d}$ denotes the identity matrix, $\underline{\underline{D}}(\vec{u}):=\frac{1}{2}\left(\nabla \vec{u}+(\nabla \vec{u})^{T}\right)$ is the rate-of-deformation tensor, $p: \Omega \times[0, T] \rightarrow \mathbb{R}$ is the pressure and $\mu(t)=\mu_{+} \mathcal{X}_{\Omega_{+}(t)}+\mu_{-} \mathcal{X}_{\Omega_{-}(t)}$, with $\mu_{ \pm} \in \mathbb{R}_{>0}$, denotes the dynamic viscosities in the two phases. On the free surface $\Gamma(t)$, the following conditions need to hold:

$$
\text { (a) }[\vec{u}]_{-}^{+}=\overrightarrow{0}, \quad \text { (b) }[\underline{\underline{\sigma}} \vec{\nu}]_{-}^{+}=-\gamma(\psi) \varkappa \vec{\nu}-\nabla_{s} \gamma(\psi), \quad \text { (c) } \overrightarrow{\mathcal{V}} \cdot \vec{\nu}=\vec{u} \cdot \vec{\nu} \quad \text { on } \Gamma(t),
$$

where $\gamma \in C^{1}\left(\left[0, \psi_{\infty}\right)\right)$, with $\psi_{\infty} \in \mathbb{R}_{>0} \cup\{\infty\}$ and

$$
\gamma^{\prime}(r) \leq 0 \quad \forall r \in\left[0, \psi_{\infty}\right),
$$

denotes the surface tension which depends on the surfactant concentration $\psi: \mathcal{G}_{T} \rightarrow\left[0, \psi_{\infty}\right)$, recall $(2.2)$, and $\nabla_{s}$ denotes the surface gradient on $\Gamma(t)$. In addition, $\varkappa$ denotes the mean curvature of $\Gamma(t)$, i.e. the sum of the principal curvatures of $\Gamma(t)$, where we have adopted the sign convention that $\varkappa$ is negative where $\Omega_{-}(t)$ is locally convex. In particular, on letting id denote the identity function in $\mathbb{R}^{d}$, it holds that

$$
\Delta_{s} \overrightarrow{\mathrm{id}}=\varkappa \vec{\nu}=: \vec{\varkappa} \quad \text { on } \Gamma(t),
$$


where $\Delta_{s}=\nabla_{s} . \nabla_{s}$ is the Laplace-Beltrami operator on $\Gamma(t)$, with $\nabla_{s}$. denoting surface divergence on $\Gamma(t)$. Moreover, as usual, $[\vec{u}]_{-}^{+}:=\vec{u}_{+}-\vec{u}_{-}$and $[\underline{\underline{\sigma}} \vec{\nu}]_{-}^{+}:=\underline{\underline{\sigma}}_{+} \vec{\nu}-\underline{\underline{\sigma}}_{-} \vec{\nu}$ denote the jumps in velocity and normal stress across the interface $\Gamma(t)$. Here and throughout, we employ the shorthand notation $\vec{g}_{ \pm}:=\left.\vec{g}\right|_{\Omega_{ \pm}(t)}$ for a function $\vec{g}: \Omega \times[0, T] \rightarrow \mathbb{R}^{d}$; and similarly for scalar and matrix-valued functions. The surfactant transport (with diffusion) on $\Gamma(t)$ is then given by

$$
\partial_{t}^{\bullet} \psi+\psi \nabla_{s} \cdot \vec{u}-\nabla_{s} \cdot\left(\mathcal{D}_{\Gamma} \nabla_{s} \psi\right)=0 \quad \text { on } \Gamma(t),
$$

where $\mathcal{D}_{\Gamma} \geq 0$ is a diffusion coefficient, and where

$$
\partial_{t}^{\bullet} \zeta=\zeta_{t}+\vec{u} . \nabla \zeta \quad \forall \zeta \in H^{1}\left(\mathcal{G}_{T}\right)
$$

denotes the material time derivative of $\zeta$ on $\Gamma(t)$. Here we stress that the derivative in (2.9) is well-defined, and depends only on the values of $\zeta$ on $\mathcal{G}_{T}$, even though $\zeta_{t}$ and $\nabla \zeta$ do not make sense separately; see e.g. [20], page 324 . The system $(2.3 \mathrm{a}-\mathrm{d}),(2.4),(2.5),(2.8)$ is closed with the initial conditions

$$
\Gamma(0)=\Gamma_{0}, \quad \psi(\cdot, 0)=\psi_{0} \quad \text { on } \Gamma_{0}, \quad \vec{u}(\cdot, 0)=\vec{u}_{0} \quad \text { in } \Omega,
$$

where $\Gamma_{0} \subset \Omega, \vec{u}_{0}: \Omega \rightarrow \mathbb{R}^{d}$, with $\nabla \cdot \vec{u}_{0}=0$, and $\psi_{0}: \Gamma_{0} \rightarrow\left[0, \psi_{\infty}\right)$ are given initial data.

For later purposes, we introduce the surface energy function $F$, which satisfies

$$
\gamma(r)=F(r)-r F^{\prime}(r) \quad \forall r \in\left(0, \psi_{\infty}\right),
$$

and

$$
\lim _{r \rightarrow 0} r F^{\prime}(r)=F(0)-\gamma(0)=0
$$

This means in particular that

$$
\gamma^{\prime}(r)=-r F^{\prime \prime}(r) \quad \forall r \in\left(0, \psi_{\infty}\right) .
$$

It immediately follows from $(2.12)$ and $(2.6)$ that $F \in C\left(\left[0, \psi_{\infty}\right)\right) \cap C^{2}\left(0, \psi_{\infty}\right)$ is convex. Typical examples for $\gamma$ and $F$ are given by

$$
\gamma(r)=\gamma_{0}(1-\beta r), \quad F(r)=\gamma_{0}[1+\beta r(\ln r-1)], \psi_{\infty}=\infty,
$$

which represents a linear equation of state, and by

$$
\gamma(r)=\gamma_{0}\left[1+\beta \psi_{\infty} \ln \left(1-\frac{r}{\psi_{\infty}}\right)\right], \quad F(r)=\gamma_{0}\left[1+\beta\left(r \ln \frac{r}{\psi_{\infty}-r}+\psi_{\infty} \ln \frac{\psi_{\infty}-r}{\psi_{\infty}}\right)\right],
$$

the so-called Langmuir equation of state, where $\gamma_{0} \in \mathbb{R}_{>0}$ and $\beta \in \mathbb{R}_{\geq 0}$ are further given parameters, where we note that the special case $\beta=0$ means that $(2.13 \mathrm{a}, \mathrm{b})$ reduce to

$$
F(r)=\gamma(r)=\gamma_{0} \in \mathbb{R}_{>0} \quad \forall r \in \mathbb{R} .
$$

Moreover, we observe that (2.13a) can be viewed as a linearization of (2.13b) in the sense that $\gamma$ in $(2.13 \mathrm{a})$ is affine, and $\gamma$ and $\gamma^{\prime}$ agree at the origin with $\gamma$ and $\gamma^{\prime}$ from (2.13b).

\subsection{Weak formulation with fluidic tangential velocity}

Before introducing our finite element approximation, we will state an appropriate weak formulation. With this in mind, we introduce the function spaces

$$
\begin{aligned}
& \mathbb{U}:=\left\{\vec{\varphi} \in\left[H^{1}(\Omega)\right]^{d}: \vec{\varphi}=\overrightarrow{0} \text { on } \partial_{1} \Omega, \vec{\varphi} . \overrightarrow{\mathrm{n}}=0 \text { a.e. on } \partial_{2} \Omega\right\}, \quad \mathbb{P}:=L^{2}(\Omega), \\
& \widehat{\mathbb{P}}:=\left\{\eta \in \mathbb{P}: \int_{\Omega} \eta \mathrm{d} \mathcal{L}^{d}=0\right\}, \quad \mathbb{V}:=L^{2}(0, T ; \mathbb{U}) \cap H^{1}\left(0, T ;\left[L^{2}(\Omega)\right]^{d}\right), \quad \mathbb{S}:=H^{1}\left(\mathcal{G}_{T}\right) .
\end{aligned}
$$


Let $(\cdot, \cdot)$ and $\langle\cdot, \cdot\rangle_{\Gamma(t)}$ denote the $L^{2}$-inner products on $\Omega$ and $\Gamma(t)$, respectively. We recall from [3] that it follows from $(2.3 \mathrm{~b}-\mathrm{d})$ and $(2.5 \mathrm{c})$ that

$$
(\rho(\vec{u} . \nabla) \vec{u}, \vec{\xi})=\frac{1}{2}\left[(\rho(\vec{u} . \nabla) \vec{u}, \vec{\xi})-(\rho(\vec{u} . \nabla) \vec{\xi}, \vec{u})-\left\langle[\rho]_{-}^{+} \vec{u} \cdot \vec{\nu}, \vec{u} \cdot \vec{\xi}\right\rangle_{\Gamma(t)}\right] \quad \forall \vec{\xi} \in\left[H^{1}(\Omega)\right]^{d}
$$

and

$$
\frac{\mathrm{d}}{\mathrm{d} t}(\rho \vec{u}, \vec{\xi})=\left(\rho \vec{u}_{t}, \vec{\xi}\right)+\left(\rho \vec{u}, \vec{\xi}_{t}\right)-\left\langle[\rho]_{-}^{+} \vec{u} \cdot \vec{\nu}, \vec{u} \cdot \vec{\xi}\right\rangle_{\Gamma(t)} \quad \forall \vec{\xi} \in \mathbb{V}
$$

respectively. Therefore, it holds that

$$
\left(\rho \vec{u}_{t}, \vec{\xi}\right)=\frac{1}{2}\left[\frac{\mathrm{d}}{\mathrm{d} t}(\rho \vec{u}, \vec{\xi})+\left(\rho \vec{u}_{t}, \vec{\xi}\right)-\left(\rho \vec{u}, \vec{\xi}_{t}\right)+\left\langle[\rho]_{-}^{+} \vec{u} \cdot \vec{\nu}, \vec{u} \cdot \vec{\xi}\right\rangle_{\Gamma(t)}\right] \quad \forall \vec{\xi} \in \mathbb{V}
$$

which on combining with (2.15) yields that

$$
\left(\rho\left[\vec{u}_{t}+(\vec{u} . \nabla) \vec{u}\right], \vec{\xi}\right)=\frac{1}{2}\left[\frac{\mathrm{d}}{\mathrm{d} t}(\rho \vec{u}, \vec{\xi})+\left(\rho \vec{u}_{t}, \vec{\xi}\right)-\left(\rho \vec{u}, \vec{\xi}_{t}\right)+(\rho,[(\vec{u} . \nabla) \vec{u}] \cdot \vec{\xi}-[(\vec{u} \cdot \nabla) \vec{\xi}] \cdot \vec{u})\right] \quad \forall \vec{\xi} \in \mathbb{V}
$$

Moreover, it holds on noting $(2.3 \mathrm{~d})$ and $(2.5 \mathrm{~b})$ that for all $\vec{\xi} \in \mathbb{U}$

$$
\int_{\Omega_{+}(t) \cup \Omega_{-}(t)}(\nabla \cdot \underline{\underline{\sigma}}) \cdot \vec{\xi} \mathrm{d} \mathcal{L}^{d}=-2(\mu \underline{\underline{D}}(\vec{u}), \underline{\underline{D}}(\vec{\xi}))+(p, \nabla \cdot \vec{\xi})+\left\langle\gamma(\psi) \varkappa \vec{\nu}+\nabla_{s} \gamma(\psi), \vec{\xi}\right\rangle_{\Gamma(t)} .
$$

Similarly to $(2.9)$ we define the following time derivative that follows the parameterization $\vec{x}(\cdot, t)$ of $\Gamma(t)$, rather than $\vec{u}$. In particular, we let

$$
\partial_{t}^{\circ} \zeta=\zeta_{t}+\overrightarrow{\mathcal{V}} \cdot \nabla \zeta \quad \forall \zeta \in \mathbb{S},
$$

recall (2.1). Here we stress once again that this definition is well-defined, even though $\zeta_{t}$ and $\nabla \zeta$ do not make sense separately for a function $\zeta \in \mathbb{S}$. On recalling (2.9) we obtain that

$$
\partial_{t}^{\circ}=\partial_{t}^{\bullet} \quad \text { if } \quad \overrightarrow{\mathcal{V}}=\vec{u} \quad \text { on } \Gamma(t) .
$$

We note that the definition (2.18) differs from the definition of $\partial^{\circ}$ in $[20]$, page 327, where $\partial^{\circ} \zeta=\zeta_{t}+(\overrightarrow{\mathcal{V}} \cdot \vec{\nu}) \vec{\nu} . \nabla \zeta$ for the "normal time derivative". It holds that

$$
\frac{\mathrm{d}}{\mathrm{d} t}\langle\chi, \zeta\rangle_{\Gamma(t)}=\left\langle\partial_{t}^{\circ} \chi, \zeta\right\rangle_{\Gamma(t)}+\left\langle\chi, \partial_{t}^{\circ} \zeta\right\rangle_{\Gamma(t)}+\left\langle\chi \zeta, \nabla_{s} \cdot \overrightarrow{\mathcal{V}}\right\rangle_{\Gamma(t)} \quad \forall \chi, \zeta \in \mathbb{S},
$$

see [20], Lemma 5.2, and that

$$
\left\langle\zeta, \nabla_{s} \cdot \vec{\eta}\right\rangle_{\Gamma(t)}+\left\langle\nabla_{s} \zeta, \vec{\eta}\right\rangle_{\Gamma(t)}=-\langle\zeta \vec{\eta}, \vec{\varkappa}\rangle_{\Gamma(t)} \quad \forall \zeta \in H^{1}(\Gamma(t)), \vec{\eta} \in\left[H^{1}(\Gamma(t))\right]^{d},
$$

see [20], Definition. 2.11. For later use we remark that it follows from (2.21) that

$$
\left\langle\gamma(\psi) \vec{\varkappa}+\nabla_{s} \gamma(\psi), \vec{\xi}\right\rangle_{\Gamma(t)}=\left\langle\gamma(\psi) \varkappa \vec{\nu}+\nabla_{s} \gamma(\psi), \vec{\xi}\right\rangle_{\Gamma(t)}=-\left\langle\gamma(\psi), \nabla_{s} \cdot \vec{\xi}\right\rangle_{\Gamma(t)} \quad \forall \vec{\xi} \in \mathbb{U} .
$$

The natural weak formulation of the system $(2.3 \mathrm{a}-\mathrm{d}),(2.4),(2.5),(2.8)$, which is based on $\overrightarrow{\mathcal{V}}=\left.\vec{u}\right|_{\Gamma(t)}$ as opposed to $(2.5 \mathrm{c})$, is then given as follows. Find $\Gamma(t)=\vec{x}(\Upsilon, t)$ for $t \in[0, T]$ with $\overrightarrow{\mathcal{V}} \in\left[L^{2}\left(\mathcal{G}_{T}\right)\right]^{d}$, and functions 
$\vec{u} \in \mathbb{V}, p \in L^{2}(0, T ; \widehat{\mathbb{P}}), \vec{\varkappa} \in\left[L^{2}\left(\mathcal{G}_{T}\right)\right]^{d}$ and $\psi \in \mathbb{S}$ such that for almost all $t \in(0, T)$ it holds that

$$
\begin{aligned}
& \frac{1}{2}\left[\frac{\mathrm{d}}{\mathrm{d} t}(\rho \vec{u}, \vec{\xi})+\left(\rho \vec{u}_{t}, \vec{\xi}\right)-\left(\rho \vec{u}, \vec{\xi}_{t}\right)+(\rho,[(\vec{u} \cdot \nabla) \vec{u}] \cdot \vec{\xi}-[(\vec{u} . \nabla) \vec{\xi}] \cdot \vec{u})\right] \\
& \quad+2(\mu \underline{\underline{D}}(\vec{u}), \underline{\underline{D}}(\vec{\xi}))-(p, \nabla \cdot \vec{\xi})-\left\langle\gamma(\psi) \vec{\varkappa}+\nabla_{s} \gamma(\psi), \vec{\xi}\right\rangle_{\Gamma(t)}=(\vec{f}, \vec{\xi}) \quad \forall \vec{\xi} \in \mathbb{V}, \\
& (\nabla \cdot \vec{u}, \varphi)=0 \quad \forall \varphi \in \widehat{\mathbb{P}} \\
& \langle\overrightarrow{\mathcal{V}}-\vec{u}, \vec{\chi}\rangle_{\Gamma(t)}=0 \quad \forall \vec{\chi} \in\left[L^{2}(\Gamma(t))\right]^{d}, \\
& \langle\vec{\varkappa}, \vec{\eta}\rangle_{\Gamma(t)}+\left\langle\nabla_{s} \overrightarrow{\mathrm{id}}, \nabla_{s} \vec{\eta}\right\rangle_{\Gamma(t)}=0 \quad \forall \vec{\eta} \in\left[H^{1}(\Gamma(t))\right]^{d}, \\
& \frac{\mathrm{d}}{\mathrm{d} t}\langle\psi, \zeta\rangle_{\Gamma(t)}+\mathcal{D}_{\Gamma}\left\langle\nabla_{s} \psi, \nabla_{s} \zeta\right\rangle_{\Gamma(t)}=\left\langle\psi, \partial_{t}^{\circ} \zeta\right\rangle_{\Gamma(t)} \quad \forall \zeta \in \mathbb{S},
\end{aligned}
$$

as well as the initial conditions (2.10), where in (2.23c) we have recalled (2.1). Here (2.23a-d) can be derived analogously to the weak formulation presented in [3], recall (2.16) and (2.17), while (2.23e) is a direct consequence of (2.20) and (2.21); see [20]. Of course, it follows from (2.23c) and (2.19) that $\partial_{t}^{\circ}$ in (2.23e) can be replaced by $\partial_{t}^{\bullet}$.

Remark 2.1. For ease of presentation, in this paper we restrict ourselves to the case of two-phase NavierStokes flow, i.e. $\rho_{ \pm}>0$. However, it is a simple matter to generalize the results in this paper to two-phase Stokes flow in the bulk, i.e. to $\rho_{+}=\rho_{-}=0$. For example, the weak formulation $(2.23 \mathrm{a}-\mathrm{e})$ then holds with $\rho=0$ and with $\mathbb{V}$ replaced by $L^{2}(0, T ; \mathbb{U})$; and analogous simplifications can be applied to the finite element approximations that will be introduced later in this paper, see also [9]. For example, the presented fully discrete schemes in Section 4 are valid for arbitrary choices of $\rho_{ \pm} \geq 0$.

\subsection{Energy bounds}

In what follows we would like to derive an energy bound for a solution of (2.23a-e). All of the following considerations are formal, in the sense that we make the appropriate assumptions about the existence, boundedness and regularity of a solution to $(2.23 \mathrm{a}-\mathrm{e})$. In particular, we assume that $\psi \in\left[0, \psi_{\infty}\right)$. Choosing $\vec{\xi}=\vec{u}$ in $(2.23 \mathrm{a})$ and $\varphi=p(\cdot, t)$ in $(2.23 \mathrm{~b})$ yields that

$$
\frac{1}{2} \frac{\mathrm{d}}{\mathrm{d} t}\left\|\rho^{\frac{1}{2}} \vec{u}\right\|_{0}^{2}+2\left\|\mu^{\frac{1}{2}} \underline{\underline{D}}(\vec{u})\right\|_{0}^{2}=(\vec{f}, \vec{u})+\left\langle\gamma(\psi) \vec{\varkappa}+\nabla_{s} \gamma(\psi), \vec{u}\right\rangle_{\Gamma(t)} .
$$

In what follows, assuming that $\gamma$ is not constant, recall (2.14), we would like to choose $\zeta=F^{\prime}(\psi)$ in $(2.23 \mathrm{e})$. As $F^{\prime}$ in general is singular at the origin, recall (2.12), we instead choose $\zeta=F^{\prime}(\psi+\alpha)$ for some $\alpha \in \mathbb{R}_{>0}$ with $\psi+\alpha<\psi_{\infty}$. Then we obtain, on recalling (2.11a) and (2.20), that

$$
\begin{gathered}
\frac{\mathrm{d}}{\mathrm{d} t}\langle F(\psi+\alpha)-\gamma(\psi+\alpha), 1\rangle_{\Gamma(t)}+\mathcal{D}_{\Gamma}\left\langle\nabla_{s}(\psi+\alpha), \nabla_{s} F^{\prime}(\psi+\alpha)\right\rangle_{\Gamma(t)} \\
=\left\langle\psi+\alpha, \partial_{t}^{\circ} F^{\prime}(\psi+\alpha)\right\rangle_{\Gamma(t)}+\alpha\left\langle F^{\prime}(\psi+\alpha), \nabla_{s} \cdot \overrightarrow{\mathcal{V}}\right\rangle_{\Gamma(t)} .
\end{gathered}
$$

Moreover, choosing $\chi=\gamma(\psi+\alpha), \zeta=1$ in (2.20), and then choosing $\vec{\eta}=\overrightarrow{\mathcal{V}}, \zeta=\gamma(\psi+\alpha)$ in (2.21) leads to

$$
\begin{aligned}
\frac{\mathrm{d}}{\mathrm{d} t}\langle\gamma(\psi+\alpha), 1\rangle_{\Gamma(t)} & =\left\langle\partial_{t}^{\circ} \gamma(\psi+\alpha), 1\right\rangle_{\Gamma(t)}+\left\langle\gamma(\psi+\alpha), \nabla_{s} \cdot \overrightarrow{\mathcal{V}}\right\rangle_{\Gamma(t)} \\
& =\left\langle\partial_{t}^{\circ} \gamma(\psi+\alpha), 1\right\rangle_{\Gamma(t)}-\left\langle\gamma(\psi+\alpha) \vec{\varkappa}+\nabla_{s} \gamma(\psi+\alpha), \overrightarrow{\mathcal{V}}\right\rangle_{\Gamma(t)} .
\end{aligned}
$$


In addition, it follows from (2.12) that

$$
\partial_{t}^{\circ} \gamma(\psi+\alpha)=\gamma^{\prime}(\psi+\alpha) \partial_{t}^{\circ} \psi=-(\psi+\alpha) F^{\prime \prime}(\psi+\alpha) \partial_{t}^{\circ} \psi=-(\psi+\alpha) \partial_{t}^{\circ} F^{\prime}(\psi+\alpha) .
$$

Combining (2.25), (2.26) and (2.27) yields that

$$
\begin{aligned}
\frac{\mathrm{d}}{\mathrm{d} t}\langle F(\psi+\alpha), 1\rangle_{\Gamma(t)}+\mathcal{D}_{\Gamma}\left\langle\nabla_{s} \mathcal{F}(\psi+\alpha), \nabla_{s} \mathcal{F}(\psi+\alpha)\right\rangle_{\Gamma(t)} \\
\quad=-\left\langle\gamma(\psi+\alpha) \vec{\varkappa}+\nabla_{s} \gamma(\psi+\alpha), \overrightarrow{\mathcal{V}}\right\rangle_{\Gamma(t)}+\alpha\left\langle F^{\prime}(\psi+\alpha), \nabla_{s} \cdot \overrightarrow{\mathcal{V}}\right\rangle_{\Gamma(t)},
\end{aligned}
$$

where, on recalling (2.12) and (2.6),

$$
\mathcal{F}(r)=\int_{0}^{r}\left[F^{\prime \prime}(y)\right]^{\frac{1}{2}} \mathrm{~d} y .
$$

Letting $\alpha \rightarrow 0$ in (2.28) yields, on recalling (2.11b), that

$$
\frac{\mathrm{d}}{\mathrm{d} t}\langle F(\psi), 1\rangle_{\Gamma(t)}+\mathcal{D}_{\Gamma}\left\langle\nabla_{s} \mathcal{F}(\psi), \nabla_{s} \mathcal{F}(\psi)\right\rangle_{\Gamma(t)}=-\left\langle\gamma(\psi) \vec{\varkappa}+\nabla_{s} \gamma(\psi), \overrightarrow{\mathcal{V}}\right\rangle_{\Gamma(t)} .
$$

We note that (2.29) is still valid in the case (2.14), on noting (2.20) and (2.22). Combining (2.29) with (2.24) implies the a priori energy equation

$$
\frac{\mathrm{d}}{\mathrm{d} t}\left(\frac{1}{2}\left\|\rho^{\frac{1}{2}} \vec{u}\right\|_{0}^{2}+\langle F(\psi), 1\rangle_{\Gamma(t)}\right)+2\left\|\mu^{\frac{1}{2}} \underline{\underline{D}}(\vec{u})\right\|_{0}^{2}+\mathcal{D}_{\Gamma}\left\langle\nabla_{s} \mathcal{F}(\psi), \nabla_{s} \mathcal{F}(\psi)\right\rangle_{\Gamma(t)}=(\vec{f}, \vec{u}) .
$$

Moreover, the volume of $\Omega_{-}(t)$ is preserved in time, i.e. the mass of each phase is conserved. To see this, choose $\vec{\chi}=\vec{\nu}$ in $(2.23 \mathrm{c})$ and $\varphi=\mathcal{X}_{\Omega_{-}(t)}$ in (2.23b) to obtain

$$
\frac{\mathrm{d}}{\mathrm{d} t} \mathcal{L}^{d}\left(\Omega_{-}(t)\right)=\langle\overrightarrow{\mathcal{V}}, \vec{\nu}\rangle_{\Gamma(t)}=\langle\vec{u}, \vec{\nu}\rangle_{\Gamma(t)}=\int_{\Omega_{-}(t)} \nabla \cdot \vec{u} \mathrm{~d} \mathcal{L}^{d}=0 .
$$

In addition, we note that it immediately follows from choosing $\zeta=1$ in $(2.23 \mathrm{e})$ that the total amount of surfactant is preserved, i.e.

$$
\frac{\mathrm{d}}{\mathrm{d} t} \int_{\Gamma(t)} \psi \mathrm{d} \mathcal{H}^{d-1}=0
$$

\subsection{Weak formulation with free tangential velocity}

It will turn out that another weak formulation of the overall system (2.3a-d), (2.4), (2.5), (2.8) will lead to finite element approximations with better mesh properties. In order to derive the weak formulation, and on recalling (2.19), we note that if we relax $\overrightarrow{\mathcal{V}}=\left.\vec{u}\right|_{\Gamma(t)}$ to

$$
\overrightarrow{\mathcal{V}} \cdot \vec{\nu}=\vec{u} \cdot \vec{\nu} \quad \text { on } \Gamma(t)
$$

as in $(2.5 \mathrm{c})$, then it holds that

$$
\partial_{t}^{\circ} \zeta=\partial_{t}^{\bullet} \zeta+(\overrightarrow{\mathcal{V}}-\vec{u}) \cdot \nabla_{s} \zeta \quad \forall \zeta \in \mathbb{S} .
$$

Our preferred finite element approximation will then be based on the following weak formulation. Find $\Gamma(t)=$ $\vec{x}(\Upsilon, t)$ for $t \in[0, T]$ with $\overrightarrow{\mathcal{V}} \in\left[L^{2}\left(\mathcal{G}_{T}\right)\right]^{d}$, and functions $\vec{u} \in \mathbb{V}, p \in L^{2}(0, T ; \widehat{\mathbb{P}}), \varkappa \in L^{2}\left(\mathcal{G}_{T}\right)$ and $\psi \in \mathbb{S}$ such that 
for almost all $t \in(0, T)$ it holds that

$$
\begin{aligned}
& \frac{1}{2}\left[\frac{\mathrm{d}}{\mathrm{d} t}(\rho \vec{u}, \vec{\xi})+\left(\rho \vec{u}_{t}, \vec{\xi}\right)-\left(\rho \vec{u}, \vec{\xi}_{t}\right)+(\rho,[(\vec{u} \cdot \nabla) \vec{u}] \cdot \vec{\xi}-[(\vec{u} . \nabla) \vec{\xi}] \cdot \vec{u})\right] \\
& \quad+2(\mu \underline{\underline{D}}(\vec{u}), \underline{\underline{D}}(\vec{\xi}))-(p, \nabla \cdot \vec{\xi})-\left\langle\gamma(\psi) \varkappa \vec{\nu}+\nabla_{s} \gamma(\psi), \vec{\xi}\right\rangle_{\Gamma(t)}=(\vec{f}, \vec{\xi}) \quad \forall \vec{\xi} \in \mathbb{V}, \\
& (\nabla \cdot \vec{u}, \varphi)=0 \quad \forall \varphi \in \widehat{\mathbb{P}}, \\
& \langle\overrightarrow{\mathcal{V}}-\vec{u}, \chi \vec{\nu}\rangle_{\Gamma(t)}=0 \quad \forall \chi \in L^{2}(\Gamma(t)), \\
& \langle\varkappa \vec{\nu}, \vec{\eta}\rangle_{\Gamma(t)}+\left\langle\nabla_{s} \overrightarrow{\mathrm{id}}, \nabla_{s} \vec{\eta}\right\rangle_{\Gamma(t)}=0 \quad \forall \vec{\eta} \in\left[H^{1}(\Gamma(t))\right]^{d}, \\
& \frac{\mathrm{d}}{\mathrm{d} t}\langle\psi, \zeta\rangle_{\Gamma(t)}+\mathcal{D}_{\Gamma}\left\langle\nabla_{s} \psi, \nabla_{s} \zeta\right\rangle_{\Gamma(t)}+\left\langle\psi(\overrightarrow{\mathcal{V}}-\vec{u}), \nabla_{s} \zeta\right\rangle_{\Gamma(t)}=\left\langle\psi, \partial_{t}^{\circ} \zeta\right\rangle_{\Gamma(t)} \quad \forall \zeta \in \mathbb{S},
\end{aligned}
$$

as well as the initial conditions $(2.10)$, where in $(2.34 \mathrm{c}, \mathrm{e})$ we have recalled $(2.1)$. The derivation of $(2.34 \mathrm{a}-\mathrm{d})$ is analogous to the derivation of $(2.23 \mathrm{a}-\mathrm{d})$, while for the formulation $(2.34 \mathrm{e})$ we note $(2.20)$ and, on recalling $(2.21)$ and (2.33), the identity

$$
\begin{aligned}
\left\langle\partial_{t}^{\circ} \psi+\psi \nabla_{s} \cdot \overrightarrow{\mathcal{V}}, \zeta\right\rangle_{\Gamma(t)} & =\left\langle\partial_{t}^{\bullet} \psi+\psi \nabla_{s} \cdot \vec{u}, \zeta\right\rangle_{\Gamma(t)}+\left\langle(\overrightarrow{\mathcal{V}}-\vec{u}) \cdot \nabla_{s} \psi+\psi \nabla_{s} \cdot(\overrightarrow{\mathcal{V}}-\vec{u}), \zeta\right\rangle_{\Gamma(t)} \\
& =\left\langle\partial_{t}^{\bullet} \psi+\psi \nabla_{s} \cdot \vec{u}, \zeta\right\rangle_{\Gamma(t)}-\left\langle\psi(\overrightarrow{\mathcal{V}}-\vec{u}), \nabla_{s} \zeta\right\rangle_{\Gamma(t)}
\end{aligned}
$$

where we have used the fact that $\langle\overrightarrow{\mathcal{V}}-\vec{u}, \psi \zeta \vec{\varkappa}\rangle_{\Gamma(t)}=0$ due to $(2.34 \mathrm{c})$. In fact, a simpler way of seeing that $(2.34 \mathrm{e})$ is consistent with $(2.23 \mathrm{e})$ is to recall that the latter holds with $\partial_{t}^{\circ}$ replaced by $\partial_{t}^{\bullet}$, and so the desired result follows immediately from (2.33).

The main differences between $(2.23 \mathrm{a}-\mathrm{e})$ and $(2.34 \mathrm{a}-\mathrm{e})$ are that for the latter the scalar curvature $\varkappa$ is sought as part of the solution, rather than $\vec{\varkappa}$, that in the latter only the normal part of $\vec{u}$ affects the evolution of the parameterization $\vec{x}$, and that as a consequence the weak formulation of the advection-diffusion has to account for the additional freedom in the tangential velocity of the interface parameterization.

Similarly to $(2.24)-(2.30)$, we can formally show that a solution to $(2.34 \mathrm{a}-\mathrm{e})$ satisfies the a priori energy bound (2.30). First of all we note that since $\vec{\varkappa}=\varkappa \vec{\nu}$, a solution to (2.34a-e) satisfies (2.24). Secondly we observe that the analogue of (2.29) has as right hand side

$$
\begin{aligned}
& -\left\langle\gamma(\psi) \vec{\varkappa}+\nabla_{s} \gamma(\psi), \overrightarrow{\mathcal{V}}\right\rangle_{\Gamma(t)}-\left\langle\psi(\overrightarrow{\mathcal{V}}-\vec{u}), \nabla_{s} F^{\prime}(\psi)\right\rangle_{\Gamma(t)} \\
& \quad=-\left\langle\gamma(\psi) \varkappa \vec{\nu}+\nabla_{s} \gamma(\psi), \overrightarrow{\mathcal{V}}\right\rangle_{\Gamma(t)}+\left\langle\nabla_{s} \gamma(\psi), \overrightarrow{\mathcal{V}}-\vec{u}\right\rangle_{\Gamma(t)}=-\left\langle\gamma(\psi) \varkappa \vec{\nu}+\nabla_{s} \gamma(\psi), \vec{u}\right\rangle_{\Gamma(t)},
\end{aligned}
$$

where we have used (2.12) and (2.34c) with $\chi=\gamma(\psi) \varkappa$. Of course, $(2.35)$ now cancels with the last term in (2.24), and so we obtain (2.30). Moreover, the properties (2.31) and (2.32) also hold.

\section{Semidiscrete finite ELement approximations}

For simplicity we consider $\Omega$ to be a polyhedral domain. Then let $\mathcal{T}^{h}$ be a regular partitioning of $\Omega$ into disjoint open simplices $o_{j}^{h}, j=1, \ldots, J_{\Omega}^{h}$. Associated with $\mathcal{T}^{h}$ are the finite element spaces

$$
S_{k}^{h}:=\left\{\chi \in C(\bar{\Omega}):\left.\chi\right|_{o} \in \mathcal{P}_{k}(o) \quad \forall o \in \mathcal{T}^{h}\right\} \subset H^{1}(\Omega), \quad k \in \mathbb{N},
$$

where $\mathcal{P}_{k}(o)$ denotes the space of polynomials of degree $k$ on $o$. We also introduce $S_{0}^{h}$, the space of piecewise constant functions on $\mathcal{T}^{h}$. Let $\left\{\varphi_{k, j}^{h}\right\}_{j=1}^{K_{k}^{h}}$ be the standard basis functions for $S_{k}^{h}, k \geq 0$. We introduce $\vec{I}_{k}^{h}:[C(\bar{\Omega})]^{d} \rightarrow\left[S_{k}^{h}\right]^{d}, k \geq 1$, the standard interpolation operators, such that $\left(\vec{I}_{k}^{h} \vec{\eta}\right)\left(\vec{p}_{k, j}^{h}\right)=\vec{\eta}\left(\vec{p}_{k, j}^{h}\right)$ for 
$j=1, \ldots, K_{k}^{h}$; where $\left\{p_{k, j}^{h}\right\}_{j=1}^{K_{k}^{h}}$ denotes the coordinates of the degrees of freedom of $S_{k}^{h}, k \geq 1$. In addition we define the standard projection operator $I_{0}^{h}: L^{1}(\Omega) \rightarrow S_{0}^{h}$, such that $\left.\left(I_{0}^{h} \eta\right)\right|_{o}=\frac{1}{\mathcal{L}^{d}(o)} \int_{o} \eta \mathrm{d} \mathcal{L}^{d}$ for all $o \in \mathcal{T}^{h}$. Our approximation to the velocity and pressure on $\mathcal{T}^{h}$ will be finite element spaces $\mathbb{U}^{h} \subset \mathbb{U}$ and $\mathbb{P}^{h}(t) \subset \mathbb{P}$. We require also the space $\widehat{\mathbb{P}}^{h}(t):=\mathbb{P}^{h}(t) \cap \widehat{\mathbb{P}}$. Based on the authors' earlier work in [3,9], we will select velocity/pressure finite element spaces that satisfy the LBB inf-sup condition (see e.g. [27], p. 114), and augment the pressure space by a single additional basis function, namely by the characteristic function of the inner phase. For the obtained spaces $\left(\mathbb{U}^{h}, \mathbb{P}^{h}(t)\right)$ we are unable to prove that they satisfy an LBB condition. The extension of the given pressure finite element space, which is an example of an XFEM approach, leads to exact volume conservation of the two phases within the finite element framework. For the non-augmented spaces we may choose, for example, the lowest order Taylor-Hood element P2-P1, the P2-P0 element or the P2-(P1+P0) element on setting $\mathbb{U}^{h}=\left[S_{2}^{h}\right]^{d} \cap \mathbb{U}$, and $\mathbb{P}^{h}=S_{1}^{h}, S_{0}^{h}$ or $S_{1}^{h}+S_{0}^{h}$, respectively. We refer to [3,9] for more details.

The parametric finite element spaces in order to approximate $\vec{x}$, as well as $\vec{\varkappa}$ and $\varkappa$ in $(2.23 \mathrm{a}-\mathrm{e})$ and $(2.34 \mathrm{a}-\mathrm{e})$, respectively, are defined as follows. Similarly to [7], let $\Gamma^{h}(t) \subset \mathbb{R}^{d}$ be a $(d-1)$-dimensional polyhedral surface, i.e. a union of non-degenerate $(d-1)$-simplices with no hanging vertices (see [16], p. 164, for $d=3$ ), approximating the closed surface $\Gamma(t)$. In particular, let $\Gamma^{h}(t)=\bigcup_{j=1}^{J_{\Gamma}} \overline{\sigma_{j}^{h}(t)}$, where $\left\{\sigma_{j}^{h}(t)\right\}_{j=1}^{J_{\Gamma}}$ is a family of mutually disjoint open $(d-1)$-simplices with vertices $\left\{\vec{q}_{k}^{h}(t)\right\}_{k=1}^{K_{\Gamma}}$. Then let

$$
\underline{V}\left(\Gamma^{h}(t)\right):=\left\{\vec{\chi} \in\left[C\left(\Gamma^{h}(t)\right)\right]^{d}:\left.\vec{\chi}\right|_{\sigma_{j}^{h}} \text { is linear } \forall j=1, \ldots, J_{\Gamma}\right\}=:\left[W\left(\Gamma^{h}(t)\right)\right]^{d} \subset\left[H^{1}\left(\Gamma^{h}(t)\right)\right]^{d},
$$

where $W\left(\Gamma^{h}(t)\right) \subset H^{1}\left(\Gamma^{h}(t)\right)$ is the space of scalar continuous piecewise linear functions on $\Gamma^{h}(t)$, with $\left\{\chi_{k}^{h}(\cdot, t)\right\}_{k=1}^{K_{\Gamma}}$ denoting the standard basis of $W\left(\Gamma^{h}(t)\right)$, i.e.

$$
\chi_{k}^{h}\left(\bar{q}_{l}^{h}(t), t\right)=\delta_{k l} \quad \forall k, l \in\left\{1, \ldots, K_{\Gamma}\right\}, \quad t \in[0, T] .
$$

For later purposes, we also introduce $\pi^{h}(t): C\left(\Gamma^{h}(t)\right) \rightarrow W\left(\Gamma^{h}(t)\right)$, the standard interpolation operator at the nodes $\left\{\vec{q}_{k}^{h}(t)\right\}_{k=1}^{K_{\Gamma}}$, and similarly $\vec{\pi}^{h}(t):\left[C\left(\Gamma^{h}(t)\right)\right]^{d} \rightarrow \underline{V}\left(\Gamma^{h}(t)\right)$.

For scalar and vector functions $\eta, \zeta$ on $\Gamma^{h}(t)$ we introduce the $L^{2}$-inner product $\langle\cdot, \cdot\rangle_{\Gamma^{h}(t)}$ over the polyhedral surface $\Gamma^{h}(t)$ as follows

$$
\langle\eta, \zeta\rangle_{\Gamma^{h}(t)}:=\int_{\Gamma^{h}(t)} \eta \cdot \zeta \mathrm{d} \mathcal{H}^{d-1}
$$

If $\eta, \zeta$ are piecewise continuous, with possible jumps across the edges of $\left\{\sigma_{j}^{h}\right\}_{j=1}^{J_{\Gamma}}$, we introduce the mass lumped inner product $\langle\cdot, \cdot\rangle_{\Gamma^{h}(t)}^{h}$ as

$$
\langle\eta, \zeta\rangle_{\Gamma^{h}(t)}^{h}:=\frac{1}{d} \sum_{j=1}^{J_{\Gamma}} \mathcal{H}^{d-1}\left(\sigma_{j}^{h}\right) \sum_{k=1}^{d}(\eta \cdot \zeta)\left(\left(\vec{q}_{j_{k}}^{h}\right)^{-}\right),
$$

where $\left\{\vec{q}_{j_{k}}^{h}\right\}_{k=1}^{d}$ are the vertices of $\sigma_{j}^{h}$, and where we define $\eta\left(\left(\vec{q}_{j_{k}}^{h}\right)^{-}\right):=\lim _{\sigma_{j}^{h} \ni \vec{p} \rightarrow \vec{q}_{j_{k}}^{h}} \eta(\vec{p})$.

On choosing an arbitrary fixed $t_{0} \in(0, T)$, we can represent each $\vec{z} \in \Gamma^{h}\left(t_{0}\right)$ as $\vec{z}=\sum_{k=1}^{K_{\Gamma}} \chi_{k}^{h}\left(\vec{z}, t_{0}\right) \vec{q}_{k}^{h}\left(t_{0}\right)$. Now we can parameterize $\Gamma^{h}(t)$ by $\vec{X}^{h}(\cdot, t): \Gamma^{h}\left(t_{0}\right) \rightarrow \mathbb{R}^{d}$, where $\vec{z} \mapsto \sum_{k=1}^{K_{\Gamma}} \chi_{k}^{h}\left(\vec{z}, t_{0}\right) \vec{q}_{k}^{h}(t)$, i.e. $\Gamma^{h}\left(t_{0}\right)$ plays the role of a reference manifold for $\left(\Gamma^{h}(t)\right)_{t \in[0, T]}$. Then, similarly to (2.1), we define the discrete velocity for $\vec{z} \in \Gamma^{h}\left(t_{0}\right)$ by

$$
\overrightarrow{\mathcal{V}}^{h}\left(\vec{z}, t_{0}\right):=\frac{\mathrm{d}}{\mathrm{d} t} \vec{X}^{h}\left(\vec{z}, t_{0}\right)=\sum_{k=1}^{K_{\Gamma}} \chi_{k}^{h}\left(\vec{z}, t_{0}\right) \frac{\mathrm{d}}{\mathrm{d} t} \vec{q}_{k}^{h}\left(t_{0}\right),
$$

which corresponds to [20], (5.23). In addition, similarly to (2.18), we define

$$
\partial_{t}^{\circ, h} \zeta\left(\vec{z}, t_{0}\right)=\frac{\mathrm{d}}{\mathrm{d} t} \zeta\left(\vec{X}^{h}\left(\vec{z}, t_{0}\right), t_{0}\right)=\zeta_{t}\left(\vec{z}, t_{0}\right)+\overrightarrow{\mathcal{V}}^{h}\left(\vec{z}, t_{0}\right) . \nabla \zeta\left(\vec{z}, t_{0}\right) \quad \forall \zeta \in H^{1}\left(\mathcal{G}_{T}^{h}\right),
$$


where, similarly to (2.2), we have defined the discrete space-time surface

$$
\mathcal{G}_{T}^{h}:=\bigcup_{t \in[0, T]} \Gamma^{h}(t) \times\{t\} .
$$

It immediately follows from (3.4) that $\partial_{t}^{\circ, h} \overrightarrow{\mathrm{id}}=\overrightarrow{\mathcal{V}}^{h}$ on $\Gamma^{h}(t)$. For later use, we also introduce the finite element spaces

$$
W\left(\mathcal{G}_{T}^{h}\right):=\left\{\chi \in C\left(\mathcal{G}_{T}^{h}\right): \chi(\cdot, t) \in W\left(\Gamma^{h}(t)\right) \quad \forall t \in[0, T]\right\}, \quad W_{T}\left(\mathcal{G}_{T}^{h}\right):=\left\{\chi \in W\left(\mathcal{G}_{T}^{h}\right): \partial_{t}^{\circ, h} \chi \in C\left(\mathcal{G}_{T}^{h}\right)\right\} .
$$

On differentiating (3.1) with respect to $t$, it immediately follows that

$$
\partial_{t}^{\circ, h} \chi_{k}^{h}=0 \quad \forall k \in\left\{1, \ldots, K_{\Gamma}\right\},
$$

see also [20], Lemma 5.5. It follows directly from (3.5) that

$$
\partial_{t}^{\circ, h} \zeta(\cdot, t)=\sum_{k=1}^{K_{\Gamma}} \chi_{k}^{h}(\cdot, t) \frac{\mathrm{d}}{\mathrm{d} t} \zeta_{k}(t) \quad \text { on } \Gamma^{h}(t)
$$

for $\zeta(\cdot, t)=\sum_{k=1}^{K_{\Gamma}} \zeta_{k}(t) \chi_{k}^{h}(\cdot, t) \in W\left(\Gamma^{h}(t)\right)$. Moreover, it holds that

$$
\frac{\mathrm{d}}{\mathrm{d} t} \int_{\sigma_{j}^{h}(t)} \zeta \mathrm{d} \mathcal{H}^{d-1}=\int_{\sigma_{j}^{h}(t)} \partial_{t}^{o, h} \zeta+\zeta \nabla_{s} \cdot \overrightarrow{\mathcal{V}}^{h} \mathrm{~d} \mathcal{H}^{d-1} \quad \forall \zeta \in H^{1}\left(\sigma_{j}^{h}(t)\right), \quad j \in\left\{1, \ldots, J_{\Gamma}\right\},
$$

see ([20], Lem. 5.6). It immediately follows from (3.7) that

$$
\frac{\mathrm{d}}{\mathrm{d} t}\langle\eta, \zeta\rangle_{\Gamma^{h}(t)}=\left\langle\partial_{t}^{\circ, h} \eta, \zeta\right\rangle_{\Gamma^{h}(t)}+\left\langle\eta, \partial_{t}^{\circ, h} \zeta\right\rangle_{\Gamma^{h}(t)}+\left\langle\eta \zeta, \nabla_{s} \cdot \overrightarrow{\mathcal{V}}^{h}\right\rangle_{\Gamma^{h}(t)} \quad \forall \eta, \zeta \in W_{T}\left(\mathcal{G}_{T}^{h}\right),
$$

which is a discrete analogue of (2.20). It is not difficult to show that the analogue of (3.8) with numerical integration also holds. We establish this result in the next lemma, together with a discrete variant of $(2.21)$, on recalling (2.7), for the case $d=2$.

Lemma 3.1. It holds that

$$
\frac{\mathrm{d}}{\mathrm{d} t}\langle\eta, \zeta\rangle_{\Gamma^{h}(t)}^{h}=\left\langle\partial_{t}^{\circ, h} \eta, \zeta\right\rangle_{\Gamma^{h}(t)}^{h}+\left\langle\eta, \partial_{t}^{\circ, h} \zeta\right\rangle_{\Gamma^{h}(t)}^{h}+\left\langle\eta \zeta, \nabla_{s} \cdot \overrightarrow{\mathcal{V}}^{h}\right\rangle_{\Gamma^{h}(t)}^{h} \quad \forall \eta, \zeta \in W_{T}\left(\mathcal{G}_{T}^{h}\right) .
$$

In addition, if $d=2$, it holds that

$$
\left\langle\zeta, \nabla_{s} \cdot \vec{\eta}\right\rangle_{\Gamma^{h}(t)}+\left\langle\nabla_{s} \zeta, \vec{\eta}\right\rangle_{\Gamma^{h}(t)}=\left\langle\nabla_{s} \overrightarrow{\mathrm{id}}, \nabla_{s} \vec{\pi}^{h}(\zeta \vec{\eta})\right\rangle_{\Gamma^{h}(t)} \quad \forall \zeta \in W\left(\Gamma^{h}(t)\right), \vec{\eta} \in \underline{V}\left(\Gamma^{h}(t)\right) .
$$

Proof. Choosing $\zeta=1$ in (3.7) yields that

$$
\frac{\mathrm{d}}{\mathrm{d} t} \mathcal{H}^{d-1}\left(\sigma_{j}^{h}(t)\right)=\mathcal{H}^{d-1}\left(\sigma_{j}^{h}(t)\right) \nabla_{s} \cdot \overrightarrow{\mathcal{V}}^{h}(\cdot, t) \quad \text { on } \sigma_{j}^{h}(t) .
$$

Differentiating (3.2) with respect to $t$, and combining with (3.11) and (3.6), yields the desired result (3.9).

For arbitrary $\zeta \in H^{1}\left(\Gamma^{h}(t)\right)$ and $\vec{\eta} \in\left[H^{1}\left(\Gamma^{h}(t)\right)\right]^{2}$ we have for $d=2$ that

$$
\left\langle\nabla_{s} \cdot(\zeta \vec{\eta}), 1\right\rangle_{\Gamma^{h}(t)}=\left\langle\overrightarrow{\mathrm{id}}_{s},(\zeta \vec{\eta})_{s}\right\rangle_{\Gamma^{h}(t)}=\left\langle\overrightarrow{\mathrm{id}}_{s},\left(\vec{\pi}^{h}[\zeta \vec{\eta}]\right)_{s}\right\rangle_{\Gamma^{h}(t)}=\left\langle\nabla_{s} \overrightarrow{\mathrm{id}}, \nabla_{s} \vec{\pi}^{h}(\zeta \vec{\eta})\right\rangle_{\Gamma^{h}(t)},
$$

which yields the desired result (3.10) on noting that $\nabla_{s} \cdot(\zeta \vec{\eta})=\zeta \nabla_{s} \cdot \vec{\eta}+\vec{\eta} \cdot \nabla_{s} \zeta$. 
Given $\Gamma^{h}(t)$, we let $\Omega_{+}^{h}(t)$ denote the exterior of $\Gamma^{h}(t)$ and let $\Omega_{-}^{h}(t)$ denote the interior of $\Gamma^{h}(t)$, so that $\Gamma^{h}(t)=\partial \Omega_{-}^{h}(t)=\overline{\Omega_{-}^{h}(t)} \cap \overline{\Omega_{+}^{h}(t)}$. We then partition the elements of the bulk mesh $\mathcal{T}^{h}$ into interior, exterior and interfacial elements as follows. Let

$$
\mathcal{T}_{-}^{h}(t):=\left\{o \in \mathcal{T}^{h}: o \subset \Omega_{-}^{h}(t)\right\}, \quad \mathcal{T}_{+}^{h}(t):=\left\{o \in \mathcal{T}^{h}: o \subset \Omega_{+}^{h}(t)\right\}, \quad \mathcal{T}_{\Gamma^{h}}^{h}(t):=\left\{o \in \mathcal{T}^{h}: o \cap \Gamma^{h}(t) \neq \emptyset\right\} .
$$

Clearly $\mathcal{T}^{h}=\mathcal{T}_{-}^{h}(t) \cup \mathcal{T}_{+}^{h}(t) \cup \mathcal{T}_{\Gamma}^{h}(t)$ is a disjoint partition. Moreover, we introduce the discrete density $\rho^{h}(t) \in S_{0}^{h}$ and the discrete viscosity $\mu^{h}(t) \in S_{0}^{h}$ as

$$
\left.\rho^{h}(t)\right|_{o}=\left\{\begin{array}{ll}
\rho_{-} & o \in \mathcal{T}_{-}^{h}(t), \\
\rho_{+} & o \in \mathcal{T}_{+}^{h}(t), \\
\frac{1}{2}\left(\rho_{-}+\rho_{+}\right) & o \in \mathcal{T}_{\Gamma^{h}}^{h}(t),
\end{array} \quad \text { and }\left.\quad \mu^{h}(t)\right|_{o}= \begin{cases}\mu_{-} & o \in \mathcal{T}_{-}^{h}(t), \\
\mu_{+} & o \in \mathcal{T}_{+}^{h}(t), \\
\frac{1}{2}\left(\mu_{-}+\mu_{+}\right) & o \in \mathcal{T}_{\Gamma^{h}}^{h}(t) .\end{cases}\right.
$$

In what follows we will introduce two different finite element approximations for the free boundary problem $(2.3 \mathrm{a}-\mathrm{d}),(2.4),(2.5),(2.8)$. Here $\vec{U}^{h}(\cdot, t) \in \mathbb{U}^{h}$ will be an approximation to $\vec{u}(\cdot, t)$, while $P^{h}(\cdot, t) \in \widehat{\mathbb{P}}^{h}(t)$ approximates $p(\cdot, t)$ and $\Psi^{h}(\cdot, t) \in W\left(\Gamma^{h}(t)\right)$ approximates $\psi(\cdot, t)$. When designing such a finite element approximation, a careful decision has to be made about the discrete tangential velocity of $\Gamma^{h}(t)$.

\subsection{Scheme $\left(A_{s d}\right)$ - approximation with fluidic tangential velocity}

The most natural choice is to select the velocity of the fluid, i.e. (2.23c) is appropriately discretized. This then gives a natural discretization of the surfactant transport equation (2.8). Note also that the approximation of curvature, recall (2.7), where now $\vec{\varkappa}=\varkappa \vec{\nu}$ is discretized directly, goes back to the seminal paper [18]. Overall, we then obtain the following semidiscrete continuous-in-time finite element approximation, which is the semidiscrete analogue of the weak formulation (2.23a-e). Given $\Gamma^{h}(0), \vec{U}^{h}(\cdot, 0) \in \mathbb{U}^{h}$ and $\Psi^{h}(\cdot, 0) \in W\left(\Gamma^{h}(0)\right)$, find $\Gamma^{h}(t)$ such that $\left.\overrightarrow{\mathrm{id}}\right|_{\Gamma^{h}(t)} \in \underline{V}\left(\Gamma^{h}(t)\right)$ for $t \in[0, T]$, and functions $\vec{U}^{h} \in H^{1}\left(0, T ; \mathbb{U}^{h}\right), P^{h} \in \mathbb{P}_{T}^{h}:=\left\{\varphi \in L^{2}(0, T ; \widehat{\mathbb{P}})\right.$ : $\varphi(t) \in \widehat{\mathbb{P}}^{h}(t)$ for a.e. $\left.t \in(0, T)\right\}, \vec{\kappa}^{h} \in\left[W\left(\mathcal{G}_{T}^{h}\right)\right]^{d}$ and $\Psi^{h} \in W_{T}\left(\mathcal{G}_{T}^{h}\right)$ such that for almost all $t \in(0, T)$ it holds that

$$
\begin{aligned}
& \frac{1}{2}\left[\frac{\mathrm{d}}{\mathrm{d} t}\left(\rho^{h} \vec{U}^{h}, \vec{\xi}\right)+\left(\rho^{h} \vec{U}_{t}^{h}, \vec{\xi}\right)-\left(\rho^{h} \vec{U}^{h}, \vec{\xi}_{t}\right)\right]+2\left(\mu^{h} \underline{\underline{D}}\left(\vec{U}^{h}\right), \underline{\underline{D}}(\vec{\xi})\right)+\frac{1}{2}\left(\rho^{h},\left[\left(\vec{U}^{h} \cdot \nabla\right) \vec{U}^{h}\right] \cdot \vec{\xi}-\left[\left(\vec{U}^{h} \cdot \nabla\right) \vec{\xi}\right] \cdot \vec{U}^{h}\right) \\
& \quad-\left(P^{h}, \nabla \cdot \vec{\xi}\right)=\left(\rho^{h} \vec{f}_{1}^{h}+\vec{f}_{2}^{h}, \vec{\xi}\right)+\left\langle\gamma\left(\Psi^{h}\right) \vec{\kappa}^{h}+\nabla_{s} \pi^{h}\left[\gamma\left(\Psi^{h}\right)\right], \vec{\xi}\right\rangle_{\Gamma^{h}(t)}^{h} \quad \forall \vec{\xi} \in H^{1}\left(0, T ; \mathbb{U}^{h}\right), \\
& \left(\nabla \cdot \vec{U}^{h}, \varphi\right)=0 \quad \forall \varphi \in \widehat{\mathbb{P}}^{h}(t), \\
& \left\langle\overrightarrow{\mathcal{V}}^{h}, \vec{\chi}\right\rangle_{\Gamma^{h}(t)}^{h}=\left\langle\vec{U}^{h}, \vec{\chi}\right\rangle_{\Gamma^{h}(t)}^{h} \quad \forall \vec{\chi} \in \underline{V}\left(\Gamma^{h}(t)\right), \\
& \left\langle\vec{\kappa}^{h}, \vec{\eta}\right\rangle_{\Gamma^{h}(t)}^{h}+\left\langle\nabla_{s} \overrightarrow{\mathrm{id}}, \nabla_{s} \vec{\eta}\right\rangle_{\Gamma^{h}(t)}=0 \quad \forall \vec{\eta} \in \underline{V}\left(\Gamma^{h}(t)\right), \\
& \frac{\mathrm{d}}{\mathrm{d} t}\left\langle\Psi^{h}, \chi\right\rangle_{\Gamma^{h}(t)}^{h}+\mathcal{D}_{\Gamma}\left\langle\nabla_{s} \Psi^{h}, \nabla_{s} \chi\right\rangle_{\Gamma^{h}(t)}=\left\langle\Psi^{h}, \partial_{t}^{\circ}, h \chi\right\rangle_{\Gamma^{h}(t)}^{h} \quad \forall \chi \in W_{T}\left(\mathcal{G}_{T}^{h}\right),
\end{aligned}
$$

where we recall (3.3). Here we have defined $\vec{f}_{i}^{h}(\cdot, t):=\vec{I}_{2}^{h} \vec{f}_{i}(\cdot, t), i=1,2$, where here and throughout we assume that $\overrightarrow{f_{i}} \in L^{2}\left(0, T ;[C(\bar{\Omega})]^{d}\right), i=1,2$. We observe that (3.12c) collapses to $\overrightarrow{\mathcal{V}}^{h}=\left.\vec{\pi}^{h} \vec{U}^{h}\right|_{\Gamma^{h}(t)} \in \underline{V}\left(\Gamma^{h}(t)\right)$, which on recalling (3.4) turns out to be crucial for the stability analysis for (3.12a-e). It is for this reason that we use mass lumping in (3.12c), which then leads to mass lumping having to be used in the last term in (3.12a), as well as for the first term in $(3.12 \mathrm{~d})$.

We remark that the formulation (3.12e) for the surfactant transport equation (2.8) falls into the framework of ESFEM (evolving surface finite element method) as coined by the authors in [19]. In this particular instance, the velocity of $\Gamma^{h}(t)$ is not a priori fixed, rather it arises implicitly through the evolution of $\Gamma^{h}(t)$ as determined 
by $(3.12 \mathrm{a}-\mathrm{e})$. Here we recall the important property (3.5), which means that (3.12e) simplifies if formulated in terms of the basis functions $\left\{\chi_{k}^{h}(\cdot, t)\right\}_{k=1}^{K_{\Gamma}}$ of $W\left(\Gamma^{h}(t)\right)$.

In the following lemma we derive a discrete analogue of (2.24).

Lemma 3.2. Let $\left\{\left(\Gamma^{h}, \vec{U}^{h}, P^{h}, \vec{\kappa}^{h}, \Psi^{h}\right)(t)\right\}_{t \in[0, T]}$ be a solution to (3.12a-e). Then

$$
\frac{1}{2} \frac{\mathrm{d}}{\mathrm{d} t}\left\|\left[\rho^{h}\right]^{\frac{1}{2}} \vec{U}^{h}\right\|_{0}^{2}+2\left\|\left[\mu^{h}\right]^{\frac{1}{2}} \underline{\underline{D}}\left(\vec{U}^{h}\right)\right\|_{0}^{2}=\left(\rho^{h} \vec{f}_{1}^{h}+\vec{f}_{2}^{h}, \vec{U}^{h}\right)+\left\langle\gamma\left(\Psi^{h}\right) \vec{\kappa}^{h}+\nabla_{s} \pi^{h}\left[\gamma\left(\Psi^{h}\right)\right], \vec{U}^{h}\right\rangle_{\Gamma^{h}(t)}^{h} .
$$

Proof. The desired result (3.13) follows immediately on choosing $\vec{\xi}=\vec{U}^{h}$ in (3.12a) and $\varphi=P^{h}$ in (3.12b).

The next theorem derives a discrete analogue of the energy law (2.30). Here, similarly to (2.25), it will be crucial to test (3.12e) with an appropriate discrete variant of $F^{\prime}\left(\Psi^{h}\right)$. It is for this reason that we have to make the following well-posedness assumption.

$$
\Psi^{h}(\cdot, t)<\psi_{\infty} \quad \text { on } \Gamma^{h}(t), \quad \forall t \in[0, T] .
$$

The theorem also establishes nonnegativity of $\Psi^{h}$ under the assumption that

$$
\int_{\sigma_{j}^{h}(t)} \nabla_{s} \chi_{i}^{h} \cdot \nabla_{s} \chi_{k}^{h} \mathrm{~d} \mathcal{H}^{d-1} \leq 0 \quad \forall i \neq k, \quad \forall t \in[0, T], \quad j=1, \ldots, J_{\Gamma} .
$$

We note that (3.15) always holds for $d=2$, and it holds for $d=3$ if all the triangles $\sigma_{i}^{h}(t)$ of $\Gamma^{h}(t)$ have no obtuse angles. A direct consequence of (3.15) is that for any monotonic function $G \in C^{0,1}(\mathbb{R})$ it holds that

$$
L_{G}\left\langle\nabla_{s} \xi, \nabla_{s} \pi^{h}[G(\xi)]\right\rangle_{\Gamma^{h}(t)} \geq\left\langle\nabla_{s} \pi^{h}[G(\xi)], \nabla_{s} \pi^{h}[G(\xi)]\right\rangle_{\Gamma^{h}(t)} \quad \forall \xi \in W\left(\Gamma^{h}(t)\right), \quad \forall t \in[0, T],
$$

where $L_{G} \in \mathbb{R}_{>0}$ denotes its Lipschitz constant. For example, (3.16) holds for $G(r)=[r]_{-}:=\min \{0, r\}$ with $L_{G}=1$.

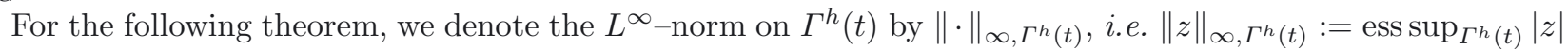
for $z: \Gamma^{h}(t) \rightarrow \mathbb{R}$.

Theorem 3.3. Let $\left\{\left(\Gamma^{h}, \vec{U}^{h}, P^{h}, \vec{\kappa}^{h}, \Psi^{h}\right)(t)\right\}_{t \in[0, T]}$ be a solution to (3.12a-e). Then

$$
\frac{\mathrm{d}}{\mathrm{d} t}\left\langle\Psi^{h}, 1\right\rangle_{\Gamma^{h}(t)}=0 .
$$

In addition, if $\mathcal{D}_{\Gamma}=0$ or if $(3.15)$ and

$$
\max _{0 \leq t \leq T}\left\|\nabla_{s} \cdot \overrightarrow{\mathcal{V}}^{h}\right\|_{\infty, \Gamma^{h}(t)}<\infty
$$

hold, then

$$
\Psi^{h}(\cdot, t) \geq 0 \quad \forall t \in(0, T] \quad \text { if } \quad \Psi^{h}(\cdot, 0) \geq 0 .
$$

Moreover, if $d=2$ and if (3.19) and (3.14) hold, then

$$
\frac{\mathrm{d}}{\mathrm{d} t}\left(\frac{1}{2}\left\|\left[\rho^{h}\right]^{\frac{1}{2}} \vec{U}^{h}\right\|_{0}^{2}+\left\langle F\left(\Psi^{h}\right), 1\right\rangle_{\Gamma^{h}(t)}^{h}\right)+2\left\|\left[\mu^{h}\right]^{\frac{1}{2}} \underline{\underline{D}}\left(\vec{U}^{h}\right)\right\|_{0}^{2} \leq\left(\rho^{h} \vec{f}_{1}^{h}+\vec{f}_{2}^{h}, \vec{U}^{h}\right) .
$$

Proof. The conservation property (3.17) follows immediately from choosing $\chi=1$ in (3.12e).

If $\mathcal{D}_{\Gamma}=0$ then it immediately follows from $(3.12 \mathrm{e})$, on recalling (3.5), that

$$
\frac{\mathrm{d}}{\mathrm{d} t}\left\langle\Psi^{h}, \chi_{k}^{h}\right\rangle_{\Gamma^{h}(t)}^{h}=\frac{\mathrm{d}}{\mathrm{d} t}\left[\left\langle 1, \chi_{k}^{h}\right\rangle_{\Gamma^{h}(t)} \Psi^{h}\left(\vec{q}_{k}^{h}(t), t\right)\right]=0,
$$


for $k=1, \ldots, K_{\Gamma}$, which yields the desired result (3.19) if $\mathcal{D}_{\Gamma}=0$. If $\mathcal{D}_{\Gamma}>0$, then choosing $\chi=\pi^{h}\left[\Psi^{h}\right]_{-}$ in (3.12e) yields, on noting (3.16) with $G=[\cdot]_{-}$and (3.9), that

$$
\begin{aligned}
\frac{\mathrm{d}}{\mathrm{d} t}\left\langle\left[\Psi^{h}\right]_{-}^{2}, 1\right\rangle_{\Gamma^{h}(t)}^{h} & =\frac{\mathrm{d}}{\mathrm{d} t}\left\langle\Psi^{h},\left[\Psi^{h}\right]_{-}\right\rangle_{\Gamma^{h}(t)}^{h} \leq\left\langle\Psi^{h}, \partial_{t}^{\circ, h} \pi^{h}\left[\Psi^{h}\right]_{-}\right\rangle_{\Gamma^{h}(t)}^{h}=\left\langle\left[\Psi^{h}\right]_{-}, \partial_{t}^{\circ, h} \pi^{h}\left[\Psi^{h}\right]_{-}\right\rangle_{\Gamma^{h}(t)}^{h} \\
& =\frac{1}{2}\left\langle\partial_{t}^{\circ, h} \pi^{h}\left[\Psi^{h}\right]_{-}^{2}, 1\right\rangle_{\Gamma^{h}(t)}^{h}=\frac{1}{2} \frac{\mathrm{d}}{\mathrm{d} t}\left\langle\left[\Psi^{h}\right]_{-}^{2}, 1\right\rangle_{\Gamma^{h}(t)}^{h}-\frac{1}{2}\left\langle\left[\Psi^{h}\right]_{-}^{2}, \nabla_{s} \cdot \overrightarrow{\mathcal{V}}^{h}\right\rangle_{\Gamma^{h}(t)}^{h} \\
& \leq-\left\langle\left[\Psi^{h}\right]_{-}^{2}, \nabla_{s} \cdot \overrightarrow{\mathcal{V}}^{h}\right\rangle_{\Gamma^{h}(t)}^{h} \leq\left\|\nabla_{s} \cdot \overrightarrow{\mathcal{V}}^{h}\right\|_{\infty, \Gamma^{h}(t)}\left\langle\left[\Psi^{h}\right]_{-}^{2}, 1\right\rangle_{\Gamma^{h}(t)}^{h} .
\end{aligned}
$$

A Gronwall inequality, together with (3.18), now yields our desired result (3.19).

For the proof of (3.20) we note that the assumption (3.14) means that we can choose $\chi=\pi^{h}\left[F^{\prime}\left(\Psi^{h}+\alpha\right)\right]$ in (3.12e), with $\alpha \in \mathbb{R}_{>0}$ such that $\Psi^{h}+\alpha<\psi_{\infty}$, to yield, on recalling (2.11a) and (3.9), that

$$
\begin{gathered}
\frac{\mathrm{d}}{\mathrm{d} t}\left\langle F\left(\Psi^{h}+\alpha\right)-\gamma\left(\Psi^{h}+\alpha\right), 1\right\rangle_{\Gamma^{h}(t)}^{h}+\mathcal{D}_{\Gamma}\left\langle\nabla_{s}\left(\Psi^{h}+\alpha\right), \nabla_{s} \pi^{h}\left[F^{\prime}\left(\Psi^{h}+\alpha\right)\right]\right\rangle_{\Gamma^{h}(t)} \\
=\left\langle\Psi^{h}+\alpha, \partial_{t}^{\circ, h} \pi^{h}\left[F^{\prime}\left(\Psi^{h}+\alpha\right)\right]\right\rangle_{\Gamma^{h}(t)}^{h}+\alpha\left\langle F^{\prime}\left(\Psi^{h}+\alpha\right), \nabla_{s} \cdot \overrightarrow{\mathcal{V}}^{h}\right\rangle_{\Gamma^{h}(t)}^{h},
\end{gathered}
$$

similarly to (2.25). For the remainder of the proof we assume that $d=2$. It follows from (2.12), (3.2) and (3.6) that we have a discrete analogue of (2.27), i.e.

$$
\left\langle\Psi^{h}+\alpha, \partial_{t}^{\circ, h} \pi^{h}\left[F^{\prime}\left(\Psi^{h}+\alpha\right)\right]\right\rangle_{\Gamma^{h}(t)}^{h}=-\left\langle\partial_{t}^{\circ, h} \pi^{h}\left[\gamma\left(\Psi^{h}+\alpha\right)\right], 1\right\rangle_{\Gamma^{h}(t)}^{h},
$$

which means that (3.21), together with (3.9), (3.10) and (3.12c,d), implies that

$$
\begin{aligned}
\frac{\mathrm{d}}{\mathrm{d} t}\langle F( & \left.\left.\Psi^{h}+\alpha\right), 1\right\rangle_{\Gamma^{h}(t)}^{h}+\mathcal{D}_{\Gamma}\left\langle\nabla_{s}\left(\Psi^{h}+\alpha\right), \nabla_{s} \pi^{h}\left[F^{\prime}\left(\Psi^{h}+\alpha\right)\right]\right\rangle_{\Gamma^{h}(t)} \\
& =\left\langle\pi^{h}\left[\gamma\left(\Psi^{h}+\alpha\right)\right], \nabla_{s} \cdot \overrightarrow{\mathcal{V}}^{h}\right\rangle_{\Gamma^{h}(t)}+\alpha\left\langle F^{\prime}\left(\Psi^{h}+\alpha\right), \nabla_{s} \cdot \overrightarrow{\mathcal{V}}^{h}\right\rangle_{\Gamma^{h}(t)}^{h} \\
& =\left\langle\nabla_{s} \overrightarrow{\mathrm{id}}, \nabla_{s} \pi^{h}\left[\gamma\left(\Psi^{h}+\alpha\right) \overrightarrow{\mathcal{V}}^{h}\right]\right\rangle_{\Gamma^{h}(t)}-\left\langle\nabla_{s} \pi^{h}\left[\gamma\left(\Psi^{h}+\alpha\right)\right], \overrightarrow{\mathcal{V}}^{h}\right\rangle_{\Gamma^{h}(t)}+\alpha\left\langle F^{\prime}\left(\Psi^{h}+\alpha\right), \nabla_{s} \cdot \overrightarrow{\mathcal{V}}^{h}\right\rangle_{\Gamma^{h}(t)}^{h} \\
& =-\left\langle\vec{\kappa}^{h}, \gamma\left(\Psi^{h}+\alpha\right) \vec{U}^{h}\right\rangle_{\Gamma^{h}(t)}^{h}-\left\langle\nabla_{s} \pi^{h}\left[\gamma\left(\Psi^{h}+\alpha\right)\right], \vec{U}^{h}\right\rangle_{\Gamma^{h}(t)}^{h}+\alpha\left\langle F^{\prime}\left(\Psi^{h}+\alpha\right), \nabla_{s} \cdot \overrightarrow{\mathcal{V}}^{h}\right\rangle_{\Gamma^{h}(t)}^{h} .
\end{aligned}
$$

Next, on noting for $\mathcal{D}_{\Gamma}>0$ that $G(\cdot)=F^{\prime}(\cdot+\alpha)$ is monotonic, as $F$ is convex, and has a finite Lipschitz constant, on noting (3.19), it follows from our assumptions and (3.16) that

$$
\mathcal{D}_{\Gamma}\left\langle\nabla_{s}\left(\Psi^{h}+\alpha\right), \nabla_{s} \pi^{h}\left[F^{\prime}\left(\Psi^{h}+\alpha\right)\right]\right\rangle_{\Gamma^{h}(t)} \geq 0,
$$

and so we obtain that

$$
\begin{aligned}
\frac{\mathrm{d}}{\mathrm{d} t}\left\langle F\left(\Psi^{h}+\alpha\right), 1\right\rangle_{\Gamma^{h}(t)}^{h} \leq & -\left\langle\vec{\kappa}^{h}, \gamma\left(\Psi^{h}+\alpha\right) \vec{U}^{h}\right\rangle_{\Gamma^{h}(t)}^{h}-\left\langle\nabla_{s} \pi^{h}\left[\gamma\left(\Psi^{h}+\alpha\right)\right], \vec{U}^{h}\right\rangle_{\Gamma^{h}(t)}^{h} \\
& +\alpha\left\langle F^{\prime}\left(\Psi^{h}+\alpha\right), \nabla_{s} \cdot \overrightarrow{\mathcal{V}}^{h}\right\rangle_{\Gamma^{h}(t)}^{h}
\end{aligned}
$$

Passing to the limit $\alpha \rightarrow 0$ in (3.25), noting (2.11b), and combining with (3.13), yields the desired result (3.20). 
Clearly, (3.17) and (3.20) are natural discrete analogues of (2.32) and (2.30), respectively.

We stress that the stability proof in Theorem 3.3 is restricted to the case $d=2$. However, it is possible to prove stability for $d=2$ and $d=3$ for a variant of $(3.12 \mathrm{a}-\mathrm{e})$, which, on recalling (2.22), is given by

$$
\begin{gathered}
\frac{1}{2}\left[\frac{\mathrm{d}}{\mathrm{d} t}\left(\rho^{h} \vec{U}^{h}, \vec{\xi}\right)+\left(\rho^{h} \vec{U}_{t}^{h}, \vec{\xi}\right)-\left(\rho^{h} \vec{U}^{h}, \vec{\xi}_{t}\right)\right]+2\left(\mu^{h} \underline{\underline{D}}\left(\vec{U}^{h}\right), \underline{\underline{D}}(\vec{\xi})\right)+\frac{1}{2}\left(\rho^{h},\left[\left(\vec{U}^{h} \cdot \nabla\right) \vec{U}^{h}\right] \cdot \vec{\xi}-\left[\left(\vec{U}^{h} \cdot \nabla\right) \vec{\xi}\right] \cdot \vec{U}^{h}\right) \\
-\left(P^{h}, \nabla \cdot \vec{\xi}\right)=\left(\rho^{h} \vec{f}_{1}^{h}+\vec{f}_{2}^{h}, \vec{\xi}\right)-\left\langle\gamma\left(\Psi^{h}\right), \nabla_{s} \cdot \vec{\pi}^{h} \vec{\xi}\right\rangle_{\Gamma^{h}(t)}^{h} \quad \forall \vec{\xi} \in H^{1}\left(0, T ; \mathbb{U}^{h}\right)
\end{gathered}
$$

together with $(3.12 \mathrm{~b}, \mathrm{c}, \mathrm{e})$. Here we observe that in this new discretization it is no longer necessary to compute the discrete curvature vector $\vec{\kappa}^{h}$. It is then not difficult to prove the following theorem.

Theorem 3.4. Let $\left\{\left(\Gamma^{h}, \vec{U}^{h}, P^{h}, \Psi^{h}\right)(t)\right\}_{t \in[0, T]}$ be a solution to (3.26), (3.12b,c,e). Then (3.17) and

$$
\frac{1}{2} \frac{\mathrm{d}}{\mathrm{d} t}\left\|\left[\rho^{h}\right]^{\frac{1}{2}} \vec{U}^{h}\right\|_{0}^{2}+2\left\|\left[\mu^{h}\right]^{\frac{1}{2}} \underline{\underline{D}}\left(\vec{U}^{h}\right)\right\|_{0}^{2}=\left(\rho^{h} \vec{f}_{1}^{h}+\vec{f}_{2}^{h}, \vec{U}^{h}\right)-\left\langle\gamma\left(\Psi^{h}\right), \nabla_{s} \cdot \vec{\pi}^{h} \vec{U}^{h}\right\rangle_{\Gamma^{h}(t)}^{h}
$$

hold. In addition, if $\mathcal{D}_{\Gamma}=0$ or if (3.15) and (3.18) hold, then we have (3.19). Moreover, if (3.14) and (3.19) hold, and $\mathcal{D}_{\Gamma}=0$ or $(3.15)$ holds, then

$$
\frac{\mathrm{d}}{\mathrm{d} t}\left(\frac{1}{2}\left\|\left[\rho^{h}\right]^{\frac{1}{2}} \vec{U}^{h}\right\|_{0}^{2}+\left\langle F\left(\Psi^{h}\right), 1\right\rangle_{\Gamma^{h}(t)}^{h}\right)+2\left\|\left[\mu^{h}\right]^{\frac{1}{2}} \underline{\underline{D}}\left(\vec{U}^{h}\right)\right\|_{0}^{2} \leq\left(\rho^{h} \vec{f}_{1}^{h}+\vec{f}_{2}^{h}, \vec{U}^{h}\right) .
$$

Proof. The desired results (3.17) and (3.27) follow immediately on choosing $\chi=1$ in (3.12e) and on choosing $\vec{\xi}=\vec{U}^{h}$ in (3.26) and $\varphi=P^{h}$ in (3.12b), respectively. The nonnegativity result (3.19) can be shown as in the proof of Theorem 3.3. The stability bound (3.28) follows as in the proof of Theorem 3.3, on combining the first equation in (3.23) with (3.27) and $\overrightarrow{\mathcal{V}}^{h}=\left.\vec{\pi}^{h} \vec{U}^{h}\right|_{\Gamma^{h}(t)}$, and on recalling that (3.24) holds if our assumptions are satisfied. We note that this proof is valid for $d=3$, as we do not use (3.10).

We recall that the assumption (3.15) always holds for $d=2$, but for $d=3$ it will in general only be satisfied if all the triangles $\sigma_{j}^{h}(t)$ of $\Gamma^{h}(t)$ have no obtuse angles. Unfortunately, in practice this will in general not be the case. Consequently, the scheme (3.26), (3.12b,c,e) offers no practical advantages over (3.12a-e), and as the latter is closer to the approximation in Section 3.2, we do not pursue (3.26), (3.12b,c,e) further in this paper.

We note that while (3.12a-e) and (3.26), (3.12b,c,e) are very natural approximations, in particular (3.12e) for the surfactant transport, see also [19], a drawback in practice is that the finitely many vertices of the triangulations $\Gamma^{h}(t)$ are moved with the flow, which can lead to coalescence. If a remeshing procedure is applied to $\Gamma^{h}(t)$, then theoretical results like stability are no longer valid. It is with this in mind that we would like to introduce an alternative finite element approximation.

\subsection{Scheme $\left(\mathrm{B}_{\mathrm{sd}}\right)$ - approximation with implicit tangential velocity}

This will be based on the weak formulation $(2.34 \mathrm{a}-\mathrm{e})$, and on the schemes from [3,9] for the two-phase flow problem in the bulk. Of course, the discretization of (2.34e) is going to be more complicated than (3.12e), but the advantage is that good mesh properties can be shown for $\Gamma^{h}(t)$. In practice this means that no remeshings or reparameterizations need to be performed for $\Gamma^{h}(t)$.

The main difference to $(3.12 \mathrm{a}-\mathrm{e})$ is that $(3.12 \mathrm{c})$ is replaced with a discrete variant of $(2.34 \mathrm{c})$. In particular, the discrete tangential velocity of $\Gamma^{h}(t)$ is not defined via $\vec{U}^{h}(\cdot, t)$, but it is chosen totally independent from the surrounding fluid. In fact, the discrete tangential velocity is not prescribed directly, but it is implicitly introduced via the novel approximation of curvature which was first introduced by the authors in [6] for the case $d=2$, and in [7] for the case $d=3$. This discrete tangential velocity is such that, in the case $d=2, \Gamma^{h}(t)$ will remain equidistributed for all times $t \in(0, T]$. For $d=3$, a weaker property can be shown, which still guarantees good meshes in practice. We refer to $[6,7]$ for more details. 
For this new finite element approximation we are unable to guarantee the nonnegativity of $\Psi^{h}(\cdot, t)$, which is in contrast to the result (3.19) for the scheme $(3.12 \mathrm{a}-\mathrm{e})$. It is for this reason that, following similar ideas in $[4,5]$, we introduce regularizations $F_{\varepsilon} \in C^{2}\left(-\infty, \psi_{\infty}\right)$ of $F \in C^{2}\left(0, \psi_{\infty}\right)$, where $\varepsilon>0$ is a regularization parameter. In particular, we set

$$
F_{\varepsilon}(r)= \begin{cases}F(r) & r \geq \varepsilon \\ F(\varepsilon)+F^{\prime}(\varepsilon)(r-\varepsilon)+\frac{1}{2} F^{\prime \prime}(\varepsilon)(r-\varepsilon)^{2} & r \leq \varepsilon\end{cases}
$$

which in view of (2.11a) leads to

$$
\gamma_{\varepsilon}(r)= \begin{cases}\gamma(r) & r \geq \varepsilon \\ \gamma(\varepsilon)+\frac{1}{2} F^{\prime \prime}(\varepsilon)\left(\varepsilon^{2}-r^{2}\right) & r \leq \varepsilon\end{cases}
$$

so that

$$
\gamma_{\varepsilon}(r)=F_{\varepsilon}(r)-r F_{\varepsilon}^{\prime}(r) \quad \text { and } \quad \gamma_{\varepsilon}^{\prime}(r)=-r F_{\varepsilon}^{\prime \prime}(r) \quad \forall r<\psi_{\infty} .
$$

We propose the following semidiscrete continuous-in-time finite element approximation, which is the semidiscrete analogue of the weak formulation $(2.34 \mathrm{a}-\mathrm{e})$. To this end, we let $\vec{\nu}^{h}(t)$ be the piecewise constant unit normal to $\Gamma^{h}(t)$ such that $\vec{\nu}^{h}(t)$ points into $\Omega_{+}^{h}(t)$. Given $\Gamma^{h}(0), \vec{U}^{h}(\cdot, 0) \in \mathbb{U}^{h}$ and $\Psi^{h}(\cdot, 0) \in W\left(\Gamma^{h}(0)\right)$, find $\Gamma^{h}(t)$ such that id $\left.\right|_{\Gamma^{h}(t)} \in \underline{V}\left(\Gamma^{h}(t)\right)$ for $t \in[0, T]$, and functions $\vec{U}^{h} \in H^{1}\left(0, T ; \mathbb{U}^{h}\right), P^{h} \in \mathbb{P}_{T}^{h}, \kappa^{h} \in W\left(\mathcal{G}_{T}^{h}\right)$ and $\Psi^{h} \in W_{T}\left(\mathcal{G}_{T}^{h}\right)$ such that for almost all $t \in(0, T)$ it holds that

$$
\begin{aligned}
& \frac{1}{2}\left[\frac{\mathrm{d}}{\mathrm{d} t}\left(\rho^{h} \vec{U}^{h}, \vec{\xi}\right)+\left(\rho^{h} \vec{U}_{t}^{h}, \vec{\xi}\right)-\left(\rho^{h} \vec{U}^{h}, \vec{\xi}_{t}\right)\right]+2\left(\mu^{h} \underline{\underline{D}}\left(\vec{U}^{h}\right), \underline{\underline{D}}(\vec{\xi})\right)+\frac{1}{2}\left(\rho^{h},\left[\left(\vec{U}^{h} \cdot \nabla\right) \vec{U}^{h}\right] \cdot \vec{\xi}-\left[\left(\vec{U}^{h} \cdot \nabla\right) \vec{\xi}\right] \cdot \vec{U}^{h}\right) \\
& \quad-\left(P^{h}, \nabla \cdot \vec{\xi}\right)=\left(\rho^{h} \vec{f}_{1}^{h}+\vec{f}_{2}^{h}, \vec{\xi}\right)+\left\langle\pi^{h}\left[\gamma_{\varepsilon}\left(\Psi^{h}\right) \kappa^{h}\right] \vec{\nu}^{h}, \vec{\xi}\right\rangle_{\Gamma^{h}(t)} \\
& \quad+\left\langle\nabla_{s} \pi^{h}\left[\gamma_{\varepsilon}\left(\Psi^{h}\right)\right], \vec{\xi}\right\rangle_{\Gamma^{h}(t)}^{h} \quad \forall \vec{\xi} \in H^{1}\left(0, T ; \mathbb{U}^{h}\right), \\
& \left(\nabla \cdot \vec{U}^{h}, \varphi\right)=0 \quad \forall \varphi \in \widehat{\mathbb{P}}^{h}(t), \\
& \left\langle\overrightarrow{\mathcal{V}}^{h}, \chi \vec{\nu}^{h}\right\rangle_{\Gamma^{h}(t)}^{h}=\left\langle\vec{U}^{h}, \chi \vec{\nu}^{h}\right\rangle_{\Gamma^{h}(t)} \quad \forall \chi \in W\left(\Gamma^{h}(t)\right), \\
& \left\langle\kappa^{h} \vec{\nu}^{h}, \vec{\eta}\right\rangle_{\Gamma^{h}(t)}^{h}+\left\langle\nabla_{s} \overrightarrow{\mathrm{i}}, \nabla_{s} \vec{\eta}\right\rangle_{\Gamma^{h}(t)}=0 \quad \forall \vec{\eta} \in \underline{V}\left(\Gamma^{h}(t)\right), \quad \text { (3.31a) } \\
& \frac{\mathrm{d}}{\mathrm{d} t}\left\langle\Psi^{h}, \chi\right\rangle_{\Gamma^{h}(t)}^{h}+\mathcal{D}_{\Gamma}\left\langle\nabla_{s} \Psi^{h}, \nabla_{s} \chi\right\rangle_{\Gamma^{h}(t)}=\left\langle\Psi^{h}, \partial_{t}^{\circ}, \chi\right\rangle_{\Gamma^{h}(t)}^{h}-\left\langle\Psi_{\star, \varepsilon}^{h}\left(\overrightarrow{\mathcal{V}}^{h}-\vec{U}^{h}\right), \nabla_{s} \chi\right\rangle_{\Gamma^{h}(t)}^{h} \quad \forall \chi \in W_{T}\left(\mathcal{G}_{T}^{h}\right),
\end{aligned}
$$

where we recall (3.3). Here $\Psi_{\star, \varepsilon}^{h}=\Psi^{h}$ for $d=3$ and, on recalling (3.30),

$$
\Psi_{\star, \varepsilon}^{h}=\left\{\begin{array}{ll}
-\frac{\gamma_{\varepsilon}\left(\Psi_{k}^{h}\right)-\gamma_{\varepsilon}\left(\Psi_{k-1}^{h}\right)}{F_{\varepsilon}^{\prime}\left(\Psi_{k}^{h}\right)-F_{\varepsilon}^{\prime}\left(\Psi_{k-1}^{h}\right)} & F_{\varepsilon}^{\prime}\left(\Psi_{k-1}^{h}\right) \neq F_{\varepsilon}^{\prime}\left(\Psi_{k}^{h}\right), \\
\frac{1}{2}\left(\Psi_{k-1}^{h}+\Psi_{k}^{h}\right) & F_{\varepsilon}^{\prime}\left(\Psi_{k-1}^{h}\right)=F_{\varepsilon}^{\prime}\left(\Psi_{k}^{h}\right),
\end{array} \quad \text { on } \quad\left[\bar{q}_{k-1}^{h}, \bar{q}_{k}^{h}\right] \quad \forall k \in\left\{1, \ldots, K_{\Gamma}\right\}\right.
$$

for $d=2$. Here we have introduced the shorthand notation $\Psi_{k}^{h}(t)=\Psi^{h}\left(\vec{q}_{k}^{h}(t), t\right)$, for $k=1, \ldots, K_{\Gamma}$, and for notational convenience we have dropped the dependence on $t$ in (3.32). The definition in (3.32) is chosen such that for $d=2$ it holds that

$$
\left\langle\Psi_{\star, \varepsilon}^{h} \vec{\eta}, \nabla_{s} \pi^{h}\left[F_{\varepsilon}^{\prime}\left(\Psi^{h}\right)\right]\right\rangle_{\Gamma^{h}(t)}^{h}=\left\langle\Psi_{\star, \varepsilon}^{h} \vec{\eta}, \nabla_{s} \pi^{h}\left[F_{\varepsilon}^{\prime}\left(\Psi^{h}\right)\right]\right\rangle_{\Gamma^{h}(t)}=-\left\langle\vec{\eta}, \nabla_{s} \pi^{h}\left[\gamma_{\varepsilon}\left(\Psi^{h}\right)\right]\right\rangle_{\Gamma^{h}(t)} \quad \forall \vec{\eta} \in \underline{V}\left(\Gamma^{h}(t)\right),
$$

which will be crucial for the stability proof for $(3.31 \mathrm{a}-\mathrm{e})$. Note that here the regularization $(3.29 \mathrm{a}, \mathrm{b})$ is required in order to make the definition (3.32) well-defined, where we recall from (2.11a) that $F^{\prime}$ in general is only 
well-defined on the positive real line. We observe that (3.33) for $\vec{\eta}=\overrightarrow{\mathcal{V}}^{h}-\left.\vec{\pi}^{h} \vec{U}^{h}\right|_{\Gamma^{h}(t)}$ mimics (2.35) on the discrete level.

Similarly to Theorem 3.3 we are only able to prove stability for the scheme (3.31a-e) in the case $d=2$. Hence in the case $d=3$ the definition (3.32) is not required, and so $\gamma_{\varepsilon}$ in (3.31a) may also be replaced by $\gamma$.

We remark that the formulation (3.31e) for the surfactant transport equation (2.8) falls into the framework of ALE ESFEM (arbitrary Lagrangian Eulerian evolving surface finite element method) as coined by the authors in [22]. In this particular instance, the tangential velocity of $\Gamma^{h}(t)$ is not a priori fixed, rather it arises implicitly through the evolution of $\Gamma^{h}(t)$ as determined by $(3.31 \mathrm{a}-\mathrm{e})$.

Similarly to Lemma 3.2 , in the following lemma we derive a discrete analogue of (2.24).

Lemma 3.5. Let $\left\{\left(\Gamma^{h}, \vec{U}^{h}, P^{h}, \kappa^{h}, \Psi^{h}\right)(t)\right\}_{t \in[0, T]}$ be a solution to (3.31a-e). Then

$$
\begin{aligned}
\frac{1}{2} \frac{\mathrm{d}}{\mathrm{d} t}\left\|\left[\rho^{h}\right]^{\frac{1}{2}} \vec{U}^{h}\right\|_{0}^{2}+2\left\|\left[\mu^{h}\right]^{\frac{1}{2}} \underline{\underline{D}}\left(\vec{U}^{h}\right)\right\|_{0}^{2} \\
\quad=\left(\rho^{h} \vec{f}_{1}^{h}+\vec{f}_{2}^{h}, \vec{U}^{h}\right)+\left\langle\pi^{h}\left[\gamma_{\varepsilon}\left(\Psi^{h}\right) \kappa^{h}\right] \vec{\nu}^{h}, \vec{U}^{h}\right\rangle_{\Gamma^{h}(t)}+\left\langle\nabla_{s} \pi^{h}\left[\gamma_{\varepsilon}\left(\Psi^{h}\right)\right], \vec{U}^{h}\right\rangle_{\Gamma^{h}(t)}^{h} .
\end{aligned}
$$

Proof. The desired result (3.34) follows immediately on choosing $\vec{\xi}=\vec{U}^{h}$ in (3.31a) and $\varphi=P^{h}$ in $(3.31 b)$.

The next theorem derives a discrete analogue of the energy law (2.30), similarly to Theorem 3.3, together with an exact volume conservation property.

Theorem 3.6. Let $\left\{\left(\Gamma^{h}, \vec{U}^{h}, P^{h}, \kappa^{h}, \Psi^{h}\right)(t)\right\}_{t \in[0, T]}$ be a solution to (3.31a-e). Then

$$
\frac{\mathrm{d}}{\mathrm{d} t}\left\langle\Psi^{h}, 1\right\rangle_{\Gamma^{h}(t)}=0 .
$$

Moreover, if $\mathcal{X}_{\Omega_{-}^{h}(t)} \in \mathbb{P}^{h}(t)$ then

$$
\frac{\mathrm{d}}{\mathrm{d} t} \mathcal{L}^{d}\left(\Omega_{-}^{h}(t)\right)=0
$$

In addition, if $d=2$ and if the assumption (3.14) holds, then

$$
\frac{\mathrm{d}}{\mathrm{d} t}\left(\frac{1}{2}\left\|\left[\rho^{h}\right]^{\frac{1}{2}} \vec{U}^{h}\right\|_{0}^{2}+\left\langle F_{\varepsilon}\left(\Psi^{h}\right), 1\right\rangle_{\Gamma^{h}(t)}^{h}\right)+2\left\|\left[\mu^{h}\right]^{\frac{1}{2}} \underline{\underline{D}}\left(\vec{U}^{h}\right)\right\|_{0}^{2} \leq\left(\rho^{h} \vec{f}_{1}^{h}+\vec{f}_{2}^{h}, \vec{U}^{h}\right) .
$$

Proof. The conservation property (3.35) follows immediately from choosing $\chi=1$ in (3.31e). Moreover, choosing $\chi=1$ in (3.31c) and $\varphi=\left(\mathcal{X}_{\Omega_{-}^{h}(t)}-\frac{\mathcal{L}^{d}\left(\Omega_{-}^{h}(t)\right)}{\mathcal{L}^{d}(\Omega)}\right) \in \widehat{\mathbb{P}}^{h}(t)$ in $(3.31 \mathrm{~b})$, we obtain that

$$
\frac{\mathrm{d}}{\mathrm{d} t} \mathcal{L}^{d}\left(\Omega_{-}^{h}(t)\right)=\left\langle\overrightarrow{\mathcal{V}}^{h}, \vec{\nu}^{h}\right\rangle_{\Gamma^{h}(t)}=\left\langle\overrightarrow{\mathcal{V}}^{h}, \vec{\nu}^{h}\right\rangle_{\Gamma^{h}(t)}^{h}=\left\langle\vec{U}^{h}, \vec{\nu}^{h}\right\rangle_{\Gamma^{h}(t)}=\int_{\Omega_{-}^{h}(t)} \nabla \cdot \vec{U}^{h} \mathrm{~d} \mathcal{L}^{d}=0,
$$

which proves the desired result (3.36). For the remainder of the proof we assume that $d=2$.

The assumption (3.14) means that we can choose $\chi=\pi^{h}\left[F_{\varepsilon}^{\prime}\left(\Psi^{h}\right)\right]$ in (3.31e) to yield, similarly to (3.21)(3.23), with $\alpha=0$ and $F$ replaced by $F_{\varepsilon}$, on recalling (3.30), (3.9), (3.10), (3.33) and (3.31c,d), that

$$
\begin{aligned}
\frac{\mathrm{d}}{\mathrm{d} t}\left\langle F_{\varepsilon}\left(\Psi^{h}\right), 1\right\rangle_{\Gamma^{h}(t)}^{h}+\mathcal{D}_{\Gamma}\left\langle\nabla_{s} \Psi^{h}, \nabla_{s} \pi^{h}\left[F_{\varepsilon}^{\prime}\left(\Psi^{h}\right)\right]\right\rangle_{\Gamma^{h}(t)} & \\
= & \left\langle\nabla_{s} \mathrm{i} \overrightarrow{\mathrm{d}}, \nabla_{s} \pi^{h}\left[\gamma_{\varepsilon}\left(\Psi^{h}\right) \overrightarrow{\mathcal{V}}^{h}\right]\right\rangle_{\Gamma^{h}(t)}-\left\langle\nabla_{s} \pi^{h}\left[\gamma_{\varepsilon}\left(\Psi^{h}\right)\right], \overrightarrow{\mathcal{V}}^{h}\right\rangle_{\Gamma^{h}(t)}+\left\langle\overrightarrow{\mathcal{V}}^{h}-\vec{\pi}^{h} \vec{U}^{h}, \nabla_{s} \pi^{h}\left[\gamma_{\varepsilon}\left(\Psi^{h}\right)\right]\right\rangle_{\Gamma^{h}(t)} \\
= & -\left\langle\kappa^{h} \vec{\nu}^{h}, \gamma_{\varepsilon}\left(\Psi^{h}\right) \overrightarrow{\mathcal{V}}^{h}\right\rangle_{\Gamma^{h}(t)}^{h}-\left\langle\vec{U}^{h}, \nabla_{s} \pi^{h}\left[\gamma_{\varepsilon}\left(\Psi^{h}\right)\right]\right\rangle_{\Gamma^{h}(t)}^{h} \\
= & -\left\langle\pi^{h}\left[\gamma_{\varepsilon}\left(\Psi^{h}\right) \kappa^{h}\right] \vec{\nu}^{h}, \vec{U}^{h}\right\rangle_{\Gamma^{h}(t)}-\left\langle\nabla_{s} \pi^{h}\left[\gamma_{\varepsilon}\left(\Psi^{h}\right)\right], \vec{U}^{h}\right\rangle_{\Gamma^{h}(t)}^{h}
\end{aligned}
$$


Since $d=2$ we can apply (3.16) to the function $G=F_{\varepsilon}^{\prime}$, where we recall $(3.14)$ and that $F_{\varepsilon} \in C^{2}\left(-\infty, \psi_{\infty}\right)$ is convex, and obtain that the second term on the left hand side of (3.38) is nonnegative. Hence the desired result (3.37) follows from combining (3.38) with (3.34).

Clearly, (3.35), (3.36) and (3.37) are natural discrete analogues of (2.32), (2.31) and (2.30), respectively. We remark that the condition $\mathcal{X}_{\Omega_{-}^{h}(t)} \in \mathbb{P}^{h}(t)$ is always satisfied for the $\mathrm{XFEM}_{\Gamma}$ approach as introduced in $[3,9]$.

In addition, it is possible to prove that the vertices of the solution $\Gamma^{h}(t)$ to $(3.31 \mathrm{a}-\mathrm{e})$ are well distributed. As this follows already from the equations $(3.31 \mathrm{~d})$, we refer to our earlier work in $[6,7]$ for further details. In particular, we observe that in the case $d=2$, i.e. for the planar two-phase problem, an equidistribution property for the vertices of $\Gamma^{h}(t)$ can be shown. These good mesh properties mean that for fully discrete schemes based on $(3.31 \mathrm{a}-\mathrm{e})$ no remeshings are required in practice for either $d=2$ or $d=3$.

We remark that for the scheme (3.12a-e) it is not possible to prove (3.36), even if mass lumping was to be dropped from the right hand side of (3.12c), because $\vec{\chi}=\vec{\nu}^{h}$ is not a valid test function in (3.12c). As a consequence, the volume of the two phases will in general not be conserved in practice. This is an additional advantage of the formulation $(3.31 \mathrm{a}-\mathrm{e})$ over $(3.12 \mathrm{a}-\mathrm{e})$. A disadvantage is the fact that it does not appear possible to derive a discrete maximum principle similarly to (3.19). However, the following remark demonstrates that also for the scheme $(3.31 \mathrm{a}-\mathrm{e})$ the negative part of $\Psi^{h}$ can be controlled. Moreover, in practice we observe that for a fully discrete variant of $(3.31 \mathrm{a}-\mathrm{e})$ the fully discrete analogues of $\Psi^{h}(\cdot, t)$ remain positive for positive initial data.

Remark 3.7. The convex nature of $F$, together with the fact that $F^{\prime}$ is singular at the origin, allows us to derive upper bounds on the negative part of $\Psi^{h}$ for the two cases (2.13a,b). On recalling (3.29a) and (2.11a), it holds that

$$
F_{\varepsilon}(r)=\gamma(\varepsilon)+F^{\prime}(\varepsilon) r+\frac{1}{2} F^{\prime \prime}(\varepsilon)(r-\varepsilon)^{2} \geq \frac{1}{2} F^{\prime \prime}(\varepsilon) r^{2} \geq \frac{1}{2} \varepsilon^{-1} \gamma_{0} \beta r^{2} \quad \forall r \leq 0,
$$

provided that $\varepsilon$ is sufficiently small. Hence the bound (3.37), via a Korn's inequality, implies that

$$
\left\langle\left[\Psi^{h}\right]_{-}^{2}, 1\right\rangle_{\Gamma^{h}(t)}^{h} \leq C \varepsilon \quad \forall t \in(0, T] \quad \text { if } \quad \Psi^{h}(\cdot, 0) \geq 0,
$$

for some positive constant $C$, and for $\varepsilon$ sufficiently small.

Remark 3.8. We note that in the special case of constant surface tension, i.e. when (2.14) holds, then, similarly to (2.29), the stability results (3.20), (3.37) and (3.28) remain valid and reduce to

$$
\frac{\mathrm{d}}{\mathrm{d} t}\left(\frac{1}{2}\left\|\left[\rho^{h}\right]^{\frac{1}{2}} \vec{U}^{h}\right\|_{0}^{2}+\gamma_{0} \mathcal{H}^{d-1}\left(\Gamma^{h}(t)\right)\right)+2\left\|\left[\mu^{h}\right]^{\frac{1}{2}} \underline{\underline{D}}\left(\vec{U}^{h}\right)\right\|_{0}^{2} \leq\left(\rho^{h} \vec{f}_{1}^{h}+\vec{f}_{2}^{h}, \vec{U}^{h}\right),
$$

where we note that $F_{\varepsilon}=F=\gamma_{0}$ in (3.37). The bound (3.39) recovers the stability results for the semidiscrete variants of the fully discrete schemes from [3] for two-phase Navier-Stokes flow.

Finally, on recalling Theorem 3.4, we remark that it does not seem possible to derive a stability result for the scheme (3.26), (3.31b-e) in the case $d=2$ or $d=3$.

\section{Fully Discrete approximations}

In this section we consider fully discrete variants of the schemes (3.12a-e) and (3.31a-e) from Section 3. Here we will choose the time discretization such that existence and uniqueness of the discrete solutions can be guaranteed, and such that we inherit as much of the structure of the stable schemes in $[3,9]$ as possible, see below for details. 
We consider the partitioning $t_{m}=m \tau, m=0, \ldots, M$, of $[0, T]$ into uniform time steps $\tau=T / M$. The time discrete spatial discretizations then directly follow from the finite element spaces introduced in Section 3, where here in order to allow for local mesh refinements we consider bulk finite element spaces that change in time.

For all $m \geq 0$, let $\mathcal{T}^{m}$ be a regular partitioning of $\Omega$ into disjoint open simplices $o_{j}^{m}, j=1, \ldots, J_{\Omega}^{m}$. We set $h^{m}:=\max _{j=1, \ldots, J_{\Omega}^{m}} \operatorname{diam}\left(o_{j}^{m}\right)$. Associated with $\mathcal{T}^{m}$ are the finite element spaces $S_{k}^{m}$ for $k \geq 0$. We introduce also $\vec{I}_{k}^{m}:[C(\bar{\Omega})]^{d} \rightarrow\left[S_{k}^{m}\right]^{d}, k \geq 1$, the standard interpolation operators, and the standard projection operator $I_{0}^{m}: L^{1}(\Omega) \rightarrow S_{0}^{m}$. For the approximation to the velocity and pressure on $\mathcal{T}^{m}$ will use the finite element spaces $\mathbb{U}^{m} \subset \mathbb{U}$ and $\mathbb{P}^{m} \subset \mathbb{P}$, which are the direct time discrete analogues of $\mathbb{U}^{h}$ and $\mathbb{P}^{h}\left(t_{m}\right)$, as well as $\widehat{\mathbb{P}}^{m} \subset \widehat{\mathbb{P}}$. We recall that $\left(\mathbb{U}^{m}, \mathbb{P}^{m}\right)$ are said to satisfy the LBB inf-sup condition if there exists a constant $C_{0} \in \mathbb{R}_{>0}$ independent of $h^{m}$ such that

$$
\inf _{\varphi \in \widehat{\mathbb{P}}^{m}} \sup _{\vec{\xi} \in \mathbb{U}^{m}} \frac{(\varphi, \nabla \cdot \vec{\xi})}{\|\varphi\|_{0}\|\vec{\xi}\|_{1}} \geq C_{0}
$$

Following the $\operatorname{XFEM}_{\Gamma}$ approach introduced in [3,9], we will often augment $\mathbb{P}^{m}$ by the single basis function $\mathcal{X}_{\Omega_{-}^{m}}$. For this extended finite element space it does not appear possible to show that (4.1) holds, but the scheme $\left(\mathrm{B}_{\mathrm{fd}}\right)$ with $\mathrm{XFEM}_{\Gamma}$ shows excellent volume conservation properties in practice; recall Theorem 3.6.

Similarly, the parametric finite element spaces are given by

$$
\underline{V}\left(\Gamma^{m}\right):=\left\{\vec{\chi} \in\left[C\left(\Gamma^{m}\right)\right]^{d}:\left.\vec{\chi}\right|_{\sigma_{j}^{m}} \text { is linear } \forall j=1, \ldots, J_{\Gamma}\right\}=:\left[W\left(\Gamma^{m}\right)\right]^{d},
$$

for $m=0, \ldots, M-1$. Here $\Gamma^{m}=\bigcup_{j=1}^{J_{\Gamma}} \overline{\sigma_{j}^{m}}$, where $\left\{\sigma_{j}^{m}\right\}_{j=1}^{J_{\Gamma}}$ is a family of mutually disjoint open $(d-1)$-simplices with vertices $\left\{\vec{q}_{k}^{m}\right\}_{k=1}^{K_{\Gamma}}$. We denote the standard basis of $W\left(\Gamma^{m}\right)$ by $\left\{\chi_{k}^{m}(\cdot, t)\right\}_{k=1}^{K_{\Gamma}}$. We also introduce $\pi^{m}$ : $C\left(\Gamma^{m}\right) \rightarrow W\left(\Gamma^{m}\right)$, the standard interpolation operator at the nodes $\left\{\vec{q}_{k}^{m}\right\}_{k=1}^{K_{\Gamma}}$, and similarly $\vec{\pi}^{m}:\left[C\left(\Gamma^{m}\right)\right]^{d} \rightarrow$ $\underline{V}\left(\Gamma^{m}\right)$. Throughout this paper, we will parameterize the new closed surface $\Gamma^{m+1}$ over $\Gamma^{m}$, with the help of a parameterization $\vec{X}^{m+1} \in \underline{V}\left(\Gamma^{m}\right)$, i.e. $\Gamma^{m+1}=\vec{X}^{m+1}\left(\Gamma^{m}\right)$.

We also introduce the $L^{2}$-inner product $\langle\cdot, \cdot\rangle_{\Gamma^{m}}$ over the current polyhedral surface $\Gamma^{m}$, as well as the the mass lumped inner product $\langle\cdot, \cdot\rangle_{\Gamma^{m}}^{h}$. Given $\Gamma^{m}$, we let $\Omega_{+}^{m}$ denote the exterior of $\Gamma^{m}$ and let $\Omega_{-}^{m}$ denote the interior of $\Gamma^{m}$, so that $\Gamma^{m}=\partial \Omega_{-}^{m}=\overline{\Omega_{-}^{m}} \cap \overline{\Omega_{+}^{m}}$. We then partition the elements of the bulk mesh $\mathcal{T}^{m}$ into interior, exterior and interfacial elements as before, and we introduce $\rho^{m}, \mu^{m} \in S_{0}^{m}$, for $m \geq 0$, as

$$
\left.\rho^{m}\right|_{o^{m}}=\left\{\begin{array}{ll}
\rho_{-} & o^{m} \in \mathcal{T}_{-}^{m}, \\
\rho_{+} & o^{m} \in \mathcal{T}_{+}^{m}, \\
\frac{1}{2}\left(\rho_{-}+\rho_{+}\right) & o^{m} \in \mathcal{T}_{\Gamma^{m}}^{m},
\end{array} \quad \text { and }\left.\quad \mu^{m}\right|_{o^{m}}= \begin{cases}\mu_{-} & o^{m} \in \mathcal{T}_{-}^{m}, \\
\mu_{+} & o^{m} \in \mathcal{T}_{+}^{m}, \\
\frac{1}{2}\left(\mu_{-}+\mu_{+}\right) & o^{m} \in \mathcal{T}_{\Gamma^{m}}^{m} .\end{cases}\right.
$$

We also set $\rho^{-1}:=\rho^{0}$.

We introduce the following pushforward operator for the discrete interfaces $\Gamma^{m-1}$ and $\Gamma^{m}$. Let $\vec{\Pi}_{m-1}^{m}$ : $\left[C\left(\Gamma^{m-1}\right)\right]^{d} \rightarrow \underline{V}\left(\Gamma^{m}\right)$ such that

$$
\left(\vec{\Pi}_{m-1}^{m} \vec{z}\right)\left(\vec{q}_{k}^{m}\right)=\vec{z}\left(\vec{q}_{k}^{m-1}\right), \quad k=1, \ldots, K_{\Gamma}, \quad \forall \vec{z} \in\left[C\left(\Gamma^{m-1}\right)\right]^{d},
$$

for $m=1, \ldots, M-1$, and set $\vec{\Pi}_{-1}^{0}:=\vec{\pi}^{0}$. Analogously to $(4.3)$ we also introduce $\Pi_{m-1}^{m}: C\left(\Gamma^{m-1}\right) \rightarrow W\left(\Gamma^{m}\right)$.

\subsection{Scheme $\left(\mathbf{A}_{\mathrm{fd}}\right)$ - approximation with fluidic tangential velocity}

Our proposed fully discrete equivalent of $(3.12 \mathrm{a}-\mathrm{e})$ is then given as follows. Let $\Gamma^{0}$, an approximation to $\Gamma(0)$, and $\vec{U}^{0} \in \mathbb{U}^{0}$, as well as $\vec{\kappa}^{0} \in \underline{V}\left(\Gamma^{0}\right)$ and $\Psi^{0} \in W\left(\Gamma^{0}\right)$ be given. For $m=0, \ldots, M-1$, find $\vec{U}^{m+1} \in \mathbb{U}^{m}$, 


$$
\begin{aligned}
& P^{m+1} \in \widehat{\mathbb{P}}^{m}, \vec{X}^{m+1} \in \underline{V}\left(\Gamma^{m}\right) \text { and } \vec{\kappa}^{m+1} \in \underline{V}\left(\Gamma^{m}\right) \text { such that } \\
& \frac{1}{2}\left(\frac{\rho^{m} \vec{U}^{m+1}-\left(I_{0}^{m} \rho^{m-1}\right) \vec{I}_{2}^{m} \vec{U}^{m}}{\tau}+\left(I_{0}^{m} \rho^{m-1}\right) \frac{\vec{U}^{m+1}-\vec{I}_{2}^{m} \vec{U}^{m}}{\tau}, \vec{\xi}\right)+2\left(\mu^{m} \underline{\underline{D}}\left(\vec{U}^{m+1}\right), \underline{\underline{D}}(\vec{\xi})\right) \\
& +\frac{1}{2}\left(\rho^{m},\left[\left(\vec{I}_{2}^{m} \vec{U}^{m} \cdot \nabla\right) \vec{U}^{m+1}\right] \cdot \vec{\xi}-\left[\left(\vec{I}_{2}^{m} \vec{U}^{m} \cdot \nabla\right) \vec{\xi}\right] \cdot \vec{U}^{m+1}\right)-\left(P^{m+1}, \nabla \cdot \vec{\xi}\right) \\
& =\left(\rho^{m} \vec{f}_{1}^{m+1}+\vec{f}_{2}^{m+1}, \vec{\xi}\right)+\left\langle\gamma\left(\Psi^{m}\right) \vec{\Pi}_{m-1}^{m} \vec{\kappa}^{m}+\nabla_{s} \pi^{m}\left[\gamma\left(\Psi^{m}\right)\right], \vec{\xi}\right\rangle_{\Gamma^{m}}^{h} \quad \forall \vec{\xi} \in \mathbb{U}^{m}, \\
& \left(\nabla \cdot \vec{U}^{m+1}, \varphi\right)=0 \quad \forall \varphi \in \widehat{\mathbb{P}}^{m}, \\
& \left\langle\frac{\vec{X}^{m+1}-\overrightarrow{\mathrm{id}}}{\tau}, \vec{\chi}\right\rangle_{\Gamma^{m}}^{h}=\left\langle\vec{U}^{m+1}, \vec{\chi}\right\rangle_{\Gamma^{m}}^{h} \quad \forall \vec{\chi} \in \underline{V}\left(\Gamma^{m}\right), \\
& \left\langle\vec{\kappa}^{m+1}, \vec{\eta}\right\rangle_{\Gamma^{m}}^{h}+\left\langle\nabla_{s} \vec{X}^{m+1}, \nabla_{s} \vec{\eta}\right\rangle_{\Gamma^{m}}=0 \quad \forall \vec{\eta} \in \underline{V}\left(\Gamma^{m}\right)
\end{aligned}
$$

and set $\Gamma^{m+1}=\vec{X}^{m+1}\left(\Gamma^{m}\right)$. Then find $\Psi^{m+1} \in W\left(\Gamma^{m+1}\right)$ such that

$$
\frac{1}{\tau}\left\langle\Psi^{m+1}, \chi_{k}^{m+1}\right\rangle_{\Gamma^{m+1}}^{h}+\mathcal{D}_{\Gamma}\left\langle\nabla_{s} \Psi^{m+1}, \nabla_{s} \chi_{k}^{m+1}\right\rangle_{\Gamma^{m+1}}=\frac{1}{\tau}\left\langle\Psi^{m}, \chi_{k}^{m}\right\rangle_{\Gamma^{m}}^{h} \quad \forall k \in\left\{1, \ldots, K_{\Gamma}\right\} .
$$

Here we have defined $\vec{f}_{i}^{m+1}:=\vec{I}_{2}^{m} \vec{f}_{i}\left(\cdot, t_{m+1}\right), i=1,2$. We observe that $(4.4 \mathrm{a}-\mathrm{e})$ is a linear scheme in that it leads to a linear system of equations for the unknowns $\left(\vec{U}^{m+1}, P^{m+1}, \vec{X}^{m+1}, \vec{\kappa}^{m+1}, \Psi^{m+1}\right)$ at each time level. In particular, the system $(4.4 \mathrm{a}-\mathrm{e})$ clearly decouples into $(4.4 \mathrm{a}, \mathrm{b})$ for $\left(\vec{U}^{m+1}, P^{m+1}\right)$, then $(4.4 \mathrm{c}, \mathrm{d})$ for $\left(\vec{X}^{m+1}, \vec{\kappa}^{m+1}\right)$ and finally $(4.4 \mathrm{e})$ for $\Psi^{m+1}$.

Remark 4.1. Of course, the natural analogue of $(4.4 \mathrm{a}-\mathrm{e})$ that is based on the semidiscrete scheme from Theorem 3.4, is given by: Find $\vec{U}^{m+1} \in \mathbb{U}^{m}, P^{m+1} \in \widehat{\mathbb{P}}^{m}, \vec{X}^{m+1} \in \underline{V}\left(\Gamma^{m}\right)$ and $\Psi^{m+1} \in W\left(\Gamma^{m+1}\right)$ such that $(4.4 \mathrm{a}-\mathrm{c}, \mathrm{e})$ hold with $\left\langle\gamma\left(\Psi^{m}\right) \vec{\kappa}^{m}+\nabla_{s} \pi^{m}\left[\gamma\left(\Psi^{m}\right)\right], \vec{\xi}\right\rangle_{\Gamma^{m}}^{h}$ in (4.4a) replaced by $-\left\langle\gamma\left(\Psi^{m}\right), \nabla_{s} \cdot \vec{\pi}^{m} \vec{\xi}_{\Gamma^{m}}^{h}\right.$.

When the velocity/pressure space pair $\left(\mathbb{U}^{m}, \widehat{\mathbb{P}}^{m}\right)$ does not satisfy $(4.1)$, we need to consider the following reduced version of $(4.4 \mathrm{a}, \mathrm{b})$, where the pressure $P^{m+1}$ is eliminated, in order to prove existence of a solution. Let

$$
\mathbb{U}_{0}^{m}:=\left\{\vec{U} \in \mathbb{U}^{m}:(\nabla \cdot \vec{U}, \varphi)=0 \quad \forall \varphi \in \widehat{\mathbb{P}}^{m}\right\} .
$$

Then any solution $\left(\vec{U}^{m+1}, P^{m+1}\right) \in \mathbb{U}^{m} \times \widehat{\mathbb{P}}^{m}$ to $(4.4 \mathrm{a}, \mathrm{b})$ is such that $\vec{U}^{m+1} \in \mathbb{U}_{0}^{m}$ satisfies (4.4a) with $\mathbb{U}^{m}$ replaced by $\mathbb{U}_{0}^{m}$. In addition, we make the following very mild well-posedness assumption.

$(\mathcal{A})$ We assume for $m=0, \ldots, M-1$ that $\mathcal{H}^{d-1}\left(\sigma_{j}^{m}\right)>0$ for all $j=1, \ldots, J_{\Gamma}$, and that $\Gamma^{m} \subset \Omega$.

Moreover, and similarly to (3.15), we note that the assumption

$$
\int_{\sigma_{j}^{m+1}} \nabla_{s} \chi_{i}^{m+1} \cdot \nabla_{s} \chi_{k}^{m+1} \mathrm{~d} \mathcal{H}^{d-1} \leq 0 \quad \forall i \neq k, \quad j=1, \ldots, J_{\Gamma}
$$

is always satisfied for $d=2$, and for $d=3$ if all the triangles $\sigma^{m+1}$ of $\Gamma^{m+1}$ have no obtuse angles.

Theorem 4.2. Let the assumption $(\mathcal{A})$ hold. If the LBB condition (4.1) holds, then there exists a unique solution $\left(\vec{U}^{m+1}, P^{m+1}\right) \in \mathbb{U}^{m} \times \widehat{\mathbb{P}}^{m}$ to $(4.4 \mathrm{a}, \mathrm{b})$. In all other cases there exists a unique solution $\vec{U}^{m+1} \in \mathbb{U}_{0}^{m}$ to the reduced equation (4.4a) with $\mathbb{U}^{m}$ replaced by $\mathbb{U}_{0}^{m}$. In either case, there exists a unique solution $\left(\vec{X}^{m+1}, \vec{\kappa}^{m+1}\right) \in$ $\underline{V}\left(\Gamma^{m}\right) \times \underline{V}\left(\Gamma^{m}\right)$ to $(4.4 \mathrm{c}, \mathrm{d})$. Finally, there exists a unique solution $\Psi^{m+1} \in W\left(\Gamma^{m+1}\right)$ to $(4.4 \mathrm{e})$ that satisfies

$$
\left\langle\Psi^{m+1}, 1\right\rangle_{\Gamma^{m+1}}=\left\langle\Psi^{m}, 1\right\rangle_{\Gamma^{m}}
$$


and, if $\mathcal{D}_{\Gamma}=0$ or if the assumption (4.5) holds,

$$
\Psi^{m+1} \geq 0 \quad \text { if } \quad \Psi^{m} \geq 0 .
$$

Proof. As all the systems are linear, existence follows from uniqueness. In order to establish the latter, we will consider the homogeneous system in each case. We begin with: Find $(\vec{U}, P) \in \mathbb{U}^{m} \times \widehat{\mathbb{P}}^{m}$ such that

$$
\begin{aligned}
& \frac{1}{2 \tau}\left(\left(\rho^{m}+I_{0}^{m} \rho^{m-1}\right) \vec{U}, \vec{\xi}\right)+2\left(\mu^{m} \underline{\underline{D}}(\vec{U}), \underline{\underline{D}}(\vec{\xi})\right)-(P, \nabla \cdot \vec{\xi}) \\
& \quad+\frac{1}{2}\left(\rho^{m},\left[\left(\vec{I}_{2}^{m} \vec{U}^{m} \cdot \nabla\right) \vec{U}\right] \cdot \vec{\xi}-\left[\left(\vec{I}_{2}^{m} \vec{U}^{m} \cdot \nabla\right) \vec{\xi}\right] \cdot \vec{U}\right)=0 \quad \forall \vec{\xi} \in \mathbb{U}^{m}, \\
& (\nabla \cdot \vec{U}, \varphi)=0 \quad \forall \varphi \in \widehat{\mathbb{P}}^{m} .
\end{aligned}
$$

Choosing $\vec{\xi}=\vec{U}$ in $(4.7 \mathrm{a})$ and $\varphi=P$ in $(4.7 \mathrm{~b})$ yields that

$$
\frac{1}{2}\left(\left(\rho^{m}+I_{0}^{m} \rho^{m-1}\right) \vec{U}, \vec{U}\right)+2 \tau\left(\mu^{m} \underline{\underline{D}}(\vec{U}), \underline{\underline{D}}(\vec{U})\right)=0 .
$$

It immediately follows from (4.8), on recalling $\rho_{ \pm}>0$, that $\vec{U}=\overrightarrow{0} \in \mathbb{U}^{m}$. Moreover, (4.7a) with $\vec{U}=\overrightarrow{0}$ implies, together with (4.1), that $P=0 \in \widehat{\mathbb{P}}^{m}$. This shows existence and uniqueness of $\left(\vec{U}^{m+1}, P^{m+1}\right) \in \mathbb{U}^{m} \times \widehat{\mathbb{P}}^{m}$. The proof for the reduced equation is very similar. The homogeneous system to consider is (4.7a) with $\mathbb{U}^{m}$ replaced by $\mathbb{U}_{0}^{m}$, where we note that the latter is a linear subspace of $\mathbb{U}^{m}$. As before, (4.8) yields that $\vec{U}=\overrightarrow{0} \in \mathbb{U}_{0}^{m}$, and so the existence of a unique solution $\vec{U}^{m+1} \in \mathbb{U}_{0}^{m}$ to the reduced equation.

Next we consider: Find $(\vec{X}, \vec{\kappa}) \in \underline{V}\left(\Gamma^{m}\right) \times \underline{V}\left(\Gamma^{m}\right)$ such that

$$
\begin{aligned}
& \langle\vec{X}, \vec{\chi}\rangle_{\Gamma^{m}}^{h}=0 \quad \forall \vec{\chi} \in \underline{V}\left(\Gamma^{m}\right), \\
& \langle\vec{\kappa}, \vec{\eta}\rangle_{\Gamma^{m}}^{h}+\left\langle\nabla_{s} \vec{X}, \nabla_{s} \vec{\eta}\right\rangle_{\Gamma^{m}}=0 \quad \forall \vec{\eta} \in \underline{V}\left(\Gamma^{m}\right),
\end{aligned}
$$

which immediately implies that $\vec{X}=\overrightarrow{0}$ and hence $\vec{\kappa}=\overrightarrow{0}$. Finally, (4.4e) is clearly a symmetric, positive definite linear system with a unique solution $\Psi^{m+1} \in W\left(\Gamma^{m+1}\right)$. The desired result (4.6a) follows on summing (4.4e) for $k=1, \ldots, K_{\Gamma}$. In order to prove (4.6b) we assume that $\Psi^{m} \geq 0$ and observe from (4.4e) that this implies that

$$
\left\langle\Psi^{m+1},\left[\Psi^{m+1}\right]_{-}\right\rangle_{\Gamma^{m+1}}^{h}+\tau \mathcal{D}_{\Gamma}\left\langle\nabla_{s} \Psi^{m+1}, \nabla_{s} \pi^{m+1}\left[\Psi^{m+1}\right]_{-}\right\rangle_{\Gamma^{m+1}} \leq 0 .
$$

Similarly to (3.16) it follows that under our assumptions the second term in (4.9) is nonnegative, which yields that

$$
\left\langle\left[\Psi^{m+1}\right]_{-},\left[\Psi^{m+1}\right]_{-}\right\rangle_{\Gamma^{m+1}}^{h}=\left\langle\Psi^{m+1},\left[\Psi^{m+1}\right]_{-}\right\rangle_{\Gamma^{m+1}}^{h} \leq 0,
$$

i.e. $\Psi^{m+1} \geq 0$.

\subsection{Scheme $\left(B_{\mathrm{fd}}\right)$ - approximation with implicit tangential velocity}

Our proposed fully discrete equivalent of $(3.31 \mathrm{a}-\mathrm{e})$ is given as follows, where we recall the regularization parameter $\varepsilon>0$ and the definitions $(3.29 \mathrm{a}, \mathrm{b})$. Let $\Gamma^{0}$, an approximation to $\Gamma(0)$, and $\vec{U}^{0} \in \mathbb{U}^{0}$, as well as $\kappa^{0} \in W\left(\Gamma^{0}\right)$ and $\Psi^{0} \in W\left(\Gamma^{0}\right)$ be given. For $m=0, \ldots, M-1$, find $\vec{U}^{m+1} \in \mathbb{U}^{m}, P^{m+1} \in \widehat{\mathbb{P}}^{m}, \vec{X}^{m+1} \in \underline{V}\left(\Gamma^{m}\right)$ 
and $\kappa^{m+1} \in W\left(\Gamma^{m}\right)$ such that

$$
\begin{aligned}
& \frac{1}{2}\left(\frac{\rho^{m} \vec{U}^{m+1}-\left(I_{0}^{m} \rho^{m-1}\right) \vec{I}_{2}^{m} \vec{U}^{m}}{\tau}+\left(I_{0}^{m} \rho^{m-1}\right) \frac{\vec{U}^{m+1}-\vec{I}_{2}^{m} \vec{U}^{m}}{\tau}, \vec{\xi}\right)+2\left(\mu^{m} \underline{\underline{D}}\left(\vec{U}^{m+1}\right), \underline{\underline{D}}(\vec{\xi})\right) \\
& \quad+\frac{1}{2}\left(\rho^{m},\left[\left(\vec{I}_{2}^{m} \vec{U}^{m} \cdot \nabla\right) \vec{U}^{m+1}\right] \cdot \vec{\xi}-\left[\left(\vec{I}_{2}^{m} \vec{U}^{m} \cdot \nabla\right) \vec{\xi}\right] \cdot \vec{U}^{m+1}\right)-\left(P^{m+1}, \nabla \cdot \vec{\xi}\right) \\
& \quad=\left(\rho^{m} \vec{f}_{1}^{m+1}+\vec{f}_{2}^{m+1}, \vec{\xi}\right)+\left\langle\pi^{m}\left[\gamma_{\varepsilon}\left(\Psi^{m}\right) \Pi_{m-1}^{m} \kappa^{m}\right] \vec{\nu}^{m}, \vec{\xi}\right\rangle_{\Gamma^{m}}+\left\langle\nabla_{s} \pi^{m}\left[\gamma_{\varepsilon}\left(\Psi^{m}\right)\right], \vec{\xi}\right\rangle_{\Gamma^{m}}^{h} \quad \forall \vec{\xi} \in \mathbb{U}^{m} \\
& \left(\nabla \cdot \vec{U}^{m+1}, \varphi\right)=0 \quad \forall \varphi \in \widehat{\mathbb{P}}^{m}, \\
& \left\langle\frac{\vec{X}^{m+1}-\overrightarrow{\mathrm{id}}}{\tau}, \chi \vec{\nu}^{m}\right\rangle_{\Gamma^{m}}^{h}=\left\langle\vec{U}^{m+1}, \chi \vec{\nu}^{m}\right\rangle_{\Gamma^{m}} \quad \forall \chi \in W\left(\Gamma^{m}\right) \\
& \left\langle\kappa^{m+1} \vec{\nu}^{m}, \vec{\eta}\right\rangle_{\Gamma^{m}}^{h}+\left\langle\nabla_{s} \vec{X}^{m+1}, \nabla_{s} \vec{\eta}\right\rangle_{\Gamma^{m}}=0 \quad \forall \vec{\eta} \in \underline{V}\left(\Gamma^{m}\right)
\end{aligned}
$$

and set $\Gamma^{m+1}=\vec{X}^{m+1}\left(\Gamma^{m}\right)$. Then find $\Psi^{m+1} \in W\left(\Gamma^{m+1}\right)$ such that

$$
\begin{aligned}
& \frac{1}{\tau}\left\langle\Psi^{m+1}, \chi_{k}^{m+1}\right\rangle_{\Gamma^{m+1}}^{h}+\mathcal{D}_{\Gamma}\left\langle\nabla_{s} \Psi^{m+1}, \nabla_{s} \chi_{k}^{m+1}\right\rangle_{\Gamma^{m+1}} \\
& \quad=\frac{1}{\tau}\left\langle\Psi^{m}, \chi_{k}^{m}\right\rangle_{\Gamma^{m}}^{h}-\left\langle\Psi_{\star, \varepsilon}^{m}\left(\frac{\vec{X}^{m+1}-\mathrm{id}}{\tau}-\vec{U}^{m+1}\right), \nabla_{s} \chi_{k}^{m}\right\rangle_{\Gamma^{m}}^{h} \quad \forall k \in\left\{1, \ldots, K_{\Gamma}\right\},
\end{aligned}
$$

where $\Psi_{\star, \varepsilon}^{m}=\Psi^{m}$ for $d=3$ and, similarly to (3.32),

$$
\Psi_{\star, \varepsilon}^{m}=\left\{\begin{array}{ll}
-\frac{\gamma_{\varepsilon}\left(\Psi_{k}^{m}\right)-\gamma_{\varepsilon}\left(\Psi_{k-1}^{m}\right)}{F_{\varepsilon}^{\prime}\left(\Psi_{k}^{m}\right)-F_{\varepsilon}^{\prime}\left(\Psi_{k-1}^{m}\right)} & F_{\varepsilon}^{\prime}\left(\Psi_{k-1}^{m}\right) \neq F_{\varepsilon}^{\prime}\left(\Psi_{k}^{m}\right), \\
\frac{1}{2}\left(\Psi_{k-1}^{m}+\Psi_{k}^{m}\right) & F_{\varepsilon}^{\prime}\left(\Psi_{k-1}^{m}\right)=F_{\varepsilon}^{\prime}\left(\Psi_{k}^{m}\right),
\end{array} \quad \text { on } \quad\left[\vec{q}_{k-1}^{m}, \vec{q}_{k}^{m}\right] \quad \forall k \in\left\{1, \ldots, K_{\Gamma}\right\}\right.
$$

for $d=2$, where $\Psi^{m}=\sum_{k=1}^{K_{\Gamma}} \Psi_{k}^{m} \chi_{k}^{m}$. We observe that (4.10a-e) is a linear scheme in that it leads to a linear system of equations for the unknowns $\left(\vec{U}^{m+1}, P^{m+1}, \vec{X}^{m+1}, \kappa^{m+1}, \Psi^{m+1}\right)$ at each time level. In particular, the system $(4.10 \mathrm{a}-\mathrm{e})$ clearly decouples into $(4.10 \mathrm{a}, \mathrm{b})$ for $\left(\vec{U}^{m+1}, P^{m+1}\right)$, then $(4.10 \mathrm{c}, \mathrm{d})$ for $\left(\vec{X}^{m+1}, \kappa^{m+1}\right)$ and finally (4.10e) for $\Psi^{m+1}$.

In order to prove the existence of a unique solution to $(4.10 \mathrm{c}, \mathrm{d})$ we need to make the following very mild additional assumption.

(B) For $k=1, \ldots, K_{\Gamma}$, let $\Xi_{k}^{m}:=\left\{\sigma_{j}^{m}: \vec{q}_{k}^{m} \in \overline{\sigma_{j}^{m}}\right\}$ and set

$$
\Lambda_{k}^{m}:=\bigcup_{\sigma_{j}^{m} \in \Xi_{k}^{m}} \overline{\sigma_{j}^{m}} \quad \text { and } \quad \vec{\omega}_{k}^{m}:=\frac{1}{\mathcal{H}^{d-1}\left(\Lambda_{k}^{m}\right)} \sum_{\sigma_{j}^{m} \in \Xi_{k}^{m}} \mathcal{H}^{d-1}\left(\sigma_{j}^{m}\right) \vec{\nu}_{j}^{m} .
$$

Then we further assume that $\operatorname{dim} \operatorname{span}\left\{\vec{\omega}_{k}^{m}\right\}_{k=1}^{K_{\Gamma}}=d, m=0, \ldots, M-1$.

We refer to [6] and [7] for more details and for an interpretation of this assumption, but we note that $(\mathcal{B})$ is always satisfied if $\Gamma^{m}$ has no self-intersections. Given the above definitions, we introduce the piecewise linear vertex normal function

$$
\vec{\omega}^{m}:=\sum_{k=1}^{K_{\Gamma}} \chi_{k}^{m} \vec{\omega}_{k}^{m} \in \underline{V}\left(\Gamma^{m}\right),
$$


and note that

$$
\left\langle\vec{v}, w \vec{\nu}^{m}\right\rangle_{\Gamma^{m}}^{h}=\left\langle\vec{v}, w \vec{\omega}^{m}\right\rangle_{\Gamma^{m}}^{h} \quad \forall \vec{v} \in \underline{V}\left(\Gamma^{m}\right), w \in W\left(\Gamma^{m}\right) .
$$

Theorem 4.3. Let the assumption $(\mathcal{A})$ hold. If the $L B B$ condition (4.1) holds, then there exists a unique solution $\left(\vec{U}^{m+1}, P^{m+1}\right) \in \mathbb{U}^{m} \times \widehat{\mathbb{P}}^{m}$ to $(4.10 \mathrm{a}, \mathrm{b})$. In all other cases there exists a unique solution $\vec{U}^{m+1} \in \mathbb{U}_{0}^{m}$ to the reduced equation (4.10a) with $\mathbb{U}^{m}$ replaced by $\mathbb{U}_{0}^{m}$. If the assumption $(\mathcal{B})$ holds, then there exists a unique solution $\left(\vec{X}^{m+1}, \kappa^{m+1}\right) \in \underline{V}\left(\Gamma^{m}\right) \times W\left(\Gamma^{m}\right)$ to $(4.10 \mathrm{c}, \mathrm{d})$. Finally, there exists a unique solution $\Psi^{m+1} \in W\left(\Gamma^{m+1}\right)$ to $(4.10 \mathrm{e})$ that satisfies (4.6a).

Proof. The results for $\vec{U}^{m+1}, P^{m+1}$ and $\Psi^{m+1}$ can be shown exactly as in the proof of Theorem 4.2. For the remaining result we consider: find $(\vec{X}, \kappa) \in \underline{V}\left(\Gamma^{m}\right) \times W\left(\Gamma^{m}\right)$ such that

$$
\begin{aligned}
& \left\langle\vec{X}, \chi \vec{\nu}^{m}\right\rangle_{\Gamma^{m}}^{h}=0 \quad \forall \chi \in W\left(\Gamma^{m}\right), \\
& \left\langle\kappa \vec{\nu}^{m}, \vec{\eta}\right\rangle_{\Gamma^{m}}^{h}+\left\langle\nabla_{s} \vec{X}, \nabla_{s} \vec{\eta}\right\rangle_{\Gamma^{m}}=0 \quad \forall \vec{\eta} \in \underline{V}\left(\Gamma^{m}\right) .
\end{aligned}
$$

Choosing $\chi=\kappa$ in (4.12a) and $\vec{\eta}=\vec{X}$ in (4.12b) yields that

$$
\left\langle\nabla_{s} \vec{X}, \nabla_{s} \vec{X}\right\rangle_{\Gamma^{m}}=0
$$

It immediately follows from (4.13) that $\vec{X}=\vec{X}_{c} \in \mathbb{R}^{d}$. Together with (4.12a), (4.11) and the assumption (B) this yields that $\vec{X}=\overrightarrow{0}$. Now (4.12b) with $\vec{\eta}=\pi^{\vec{m}}\left[\kappa \omega^{\vec{m}}\right]$, recall (4.11), implies that $\kappa=0$.

Remark 4.4. On replacing $\kappa^{m}$ in (4.10a) with $\kappa^{m+1}$ the subsystem (4.10a-d) no longer decouples. However, this system, for the special case of constant surface tension, as in (2.14), i.e. for a two-phase flow problem without surfactants, has been considered by the authors in [3]. For this finite element approximation of twophase flow, the authors proved the existence of a unique solution $\left(\vec{U}^{m+1}, \vec{X}^{m+1}, \kappa^{m+1}\right) \in \mathbb{U}_{0}^{m} \times \underline{V}\left(\Gamma^{m}\right) \times W\left(\Gamma^{m}\right)$ to the reduced system $(4.10 \mathrm{a}, \mathrm{c}, \mathrm{d})$, with $\mathbb{U}^{m}$ replaced by $\mathbb{U}_{0}^{m}$, and with $\kappa^{m}$ in $(4.10 \mathrm{a})$ replaced by $\kappa^{m+1}$, which in addition satisfies the following stability bound:

$$
\begin{aligned}
& \frac{1}{2}\left(\rho^{m} \vec{U}^{m+1}, \vec{U}^{m+1}\right)+\gamma_{0} \mathcal{H}^{d-1}\left(\Gamma^{m+1}\right)+\frac{1}{2}\left(\left(I_{0}^{m} \rho^{m-1}\right)\left(\vec{U}^{m+1}-\vec{I}_{2}^{m} \vec{U}^{m}\right), \vec{U}^{m+1}-\vec{I}_{2}^{m} \vec{U}^{m}\right) \\
& \quad+2 \tau\left(\mu^{m} \underline{\underline{D}}\left(U^{m+1}\right), \underline{\underline{D}}\left(U^{m+1}\right)\right) \leq \frac{1}{2}\left(I_{0}^{m} \rho^{m-1} \vec{I}_{2}^{m} \vec{U}^{m}, \vec{I}_{2}^{m} \vec{U}^{m}\right)+\gamma_{0} \mathcal{H}^{d-1}\left(\Gamma^{m}\right)+\tau\left(\rho^{m} \vec{f}_{1}^{m+1}+\vec{f}_{2}^{m+1}, \vec{U}^{m+1}\right) .
\end{aligned}
$$

The same stability result, in the case (2.14), can be shown for the scheme (4.4a-e), once again on replacing $\vec{\kappa}^{m}$ in $(4.4 \mathrm{a})$ with $\vec{\kappa}^{m+1}$.

The above remark motivates our choice of time discretizations in (4.10a-d). As it does not appear possible to prove a stability result similar to (3.37) for the fully discrete scheme (4.10a-e) for general choices of $\gamma$ such as $(2.13 \mathrm{a}, \mathrm{b})$, we prefer to use $\kappa^{m}$ in (4.10a) rather than $\kappa^{m+1}$, which simplifies the existence and uniqueness proof, as well as the solution procedure.

Remark 4.5. For ease of presentation we have assumed so far that the number of vertices, $K_{\Gamma}$, and the number of elements, $J_{\Gamma}$, of the discrete interface $\Gamma^{m}$ remain constant over time. However, it is a simple matter to allow for a localized refinement procedure as employed in [3]. Here any newly introduced basis function for $\Gamma^{m+1}$, say, needs to be traced back to $\Gamma^{m}$ so that (4.10e), and similarly (4.4e), remain well-defined. 


\section{NumericAl RESUlts}

For details on the assembly of the linear system arising at each time step of (4.10a-e), as well as details on the adaptive mesh refinement algorithm and the solution procedure, we refer to [3]. The main new ingredient is $(4.10 \mathrm{e})$, which decouples from $(4.10 \mathrm{a}-\mathrm{d})$ and so is straightforward to solve. In fact, the computational effort for the solution of (4.10e) is negligible compared to the two-phase flow solver for (4.10a-d). An analogous comment holds for the scheme $(4.4 \mathrm{a}-\mathrm{e})$. The schemes are implemented with the help of the finite element toolbox ALBERTA, see [36]. Note that the evaluation and the assembly of interface-bulk cross terms, such as the right hand side in $(4.10 \mathrm{c})$, is an independent software development by the authors, which is not available as a built-in capability in ALBERTA. Details on these developments can be found in $[3,8,10]$. Moreover, we recall from [3] that for the bulk mesh adaptation we use a strategy that results in a fine mesh size $h_{f}$ around $\Gamma^{m}$ and a coarse mesh size $h_{c}$ further away from it. Here $h_{f}=\frac{2 \min \left\{H_{1}, H_{2}\right\}}{N_{f}}$ and $h_{c}=\frac{2 \min \left\{H_{1}, H_{2}\right\}}{N_{c}}$ are given by two integer numbers $N_{f}>N_{c}$, where we assume from now on that $\Omega$ is given by $\times_{i=1}^{d}\left(-H_{i}, H_{i}\right)$.

For the scheme $\left(\mathrm{B}_{\mathrm{fd}}\right)$ we fix $\varepsilon=10^{-5}$, and in all our numerical experiments presented in this section the discrete surfactant concentration $\Psi^{m}$ remained above $\varepsilon$ throughout the evolution, so that $\gamma_{\varepsilon}\left(\Psi^{m}\right)=\gamma\left(\Psi^{m}\right)$, recall (3.29b). Unless otherwise stated we use the linear equation of state (2.13a) for the surface tension, and for the numerical simulations without surfactant we set $\beta=0$ in (2.13a). We set $\Psi^{0}=\psi_{0}=1$, unless stated otherwise. In addition, we employ the lowest order Taylor-Hood element P2-P1, together with the $\mathrm{XFEM}_{\Gamma}$ extension from $[3,9]$, in all computations and set $\overrightarrow{U^{0}}=\overrightarrow{I_{2}^{0}} \overrightarrow{u_{0}}$, where $\overrightarrow{u_{0}}=\overrightarrow{0}$ unless stated otherwise. For the initial interface we always choose a circle/sphere of radius $R_{0}$ and set $\kappa^{0}=-\frac{d-1}{R_{0}}$ for the scheme $\left(\mathrm{B}_{\mathrm{fd}}\right)$. For the scheme $\left(\mathrm{A}_{\mathrm{fd}}\right)$ we let $\vec{\kappa}^{0} \in \underline{V}\left(\Gamma^{0}\right)$ be the solution of (4.4d) with $m$ and $m+1$ replaced by zero. To summarize the discretization parameters we use the shorthand notation $n$ adapt $_{k, l}$ from [3]. The subscripts refer to the fineness of the spatial discretizations, i.e. for the set $n$ adapt $_{k, l}$ it holds that $N_{f}=2^{k}$ and $N_{c}=2^{l}$. For the case $d=2$ we have in addition that $K_{\Gamma}=J_{\Gamma}=2^{k}$, while for $d=3$ it holds that $\left(K_{\Gamma}, J_{\Gamma}\right)=(770,1536),(1538,3072),(3074,6144)$ for $k=5,6,7$. Finally, the uniform time step size for the set $n$ adapt $_{k, l}$ is given by $\tau=10^{-3} / n$, and if $n=1$ we

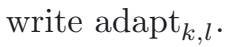

\subsection{Convergence experiments for convection diffusion equation}

In this subsection we test the two approximations $(4.4 \mathrm{c}, \mathrm{e})$ and $(4.10 \mathrm{c}-\mathrm{e})$ for the convection diffusion equation (2.8), in a situation where the evolution of the surface $\Gamma(t)$ is given. In particular, we perform convergence experiments for the true solution from the Appendix; that is, $\psi(\vec{z}, t)=e^{-6 t} z_{1} z_{2}$ is fixed on the moving ellipsoid $\Gamma(t)$ with time dependent $x_{1}$-axis. To this end, we replace $\vec{U}^{m+1}$ in $(4.4 \mathrm{c})$ and $(4.10 \mathrm{c}, \mathrm{e})$ with $\vec{u}\left(\cdot, t_{m+1}\right)$ as defined in (A.2), and set $\mathcal{D}_{\Gamma}=1$. In addition, we add the term

$$
\left\langle f_{\Gamma}^{m+1}, \chi_{k}^{m+1}\right\rangle_{\Gamma^{m+1}}^{h}
$$

to the right hand sides of (4.4e) and (4.10e), where $f_{\Gamma}^{m+1} \in W\left(\Gamma^{m+1}\right)$ is defined such that

$$
f_{\Gamma}^{m+1}\left(\vec{q}_{k}^{m+1}\right)=f_{\Gamma}\left(\vec{\Pi}_{\Gamma\left(t_{m+1}\right)} \vec{q}_{k}^{m+1}, t_{m+1}\right) \quad k=1, \ldots, K_{\Gamma},
$$

with $f_{\Gamma}$ given as in (A.3), and with $\vec{\Pi}_{\Gamma(t)}: \mathbb{R}^{d} \rightarrow \Gamma(t)$ denoting the orthogonal projection onto $\Gamma(t)$ for $t \in[0, T]$. In practice this projection can be computed with the help of a Newton iteration. In Table 1 we report on the error

$$
\|\Psi-\psi\|_{L^{2}}:=\left[\sum_{m=1}^{M} \tau\left\langle\left[\Psi^{m}-\psi\left(\cdot, t_{m}\right) \circ \vec{\Pi}_{\Gamma\left(t_{m}\right)}\right]^{2}, 1\right\rangle_{\Gamma^{m}}^{h}\right]^{\frac{1}{2}}
$$

for convergence experiments for $d=2$ and $d=3$, respectively. Here we choose the time interval $[0, T]$ with $T=1$, and for the uniform time step size we take $\tau=h_{0}^{2}$, where $h_{0}$ denotes the maximal element diameter of $\Gamma^{0}$. Of course, for the last time step we use the time step size $T-t_{M-1}=T-(M-1) \tau$. We observe that both schemes show very similar errors, indicating a convergence order of at least $\mathcal{O}\left(h_{0}^{2}\right)$. 

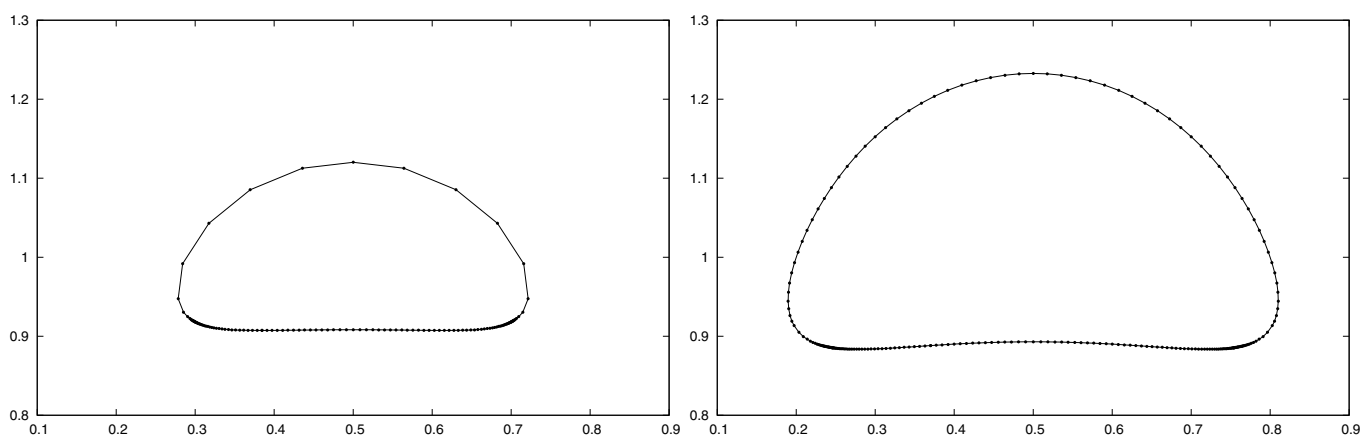

FiguRE 2. (adapt ${ }_{7,3}$ ) Vertex distributions for the final bubbles for the benchmark problem 1 at time $T=3$ for the scheme $\left(\mathrm{A}_{\mathrm{fd}}\right)$ without local refinement on $\Gamma^{m}$ (left), and with local refinement (right).

TABLE 1 . The scaled errors $\|\Psi-\psi\|_{L^{2}} / h_{0}^{2}$ for the convergence experiment for $d=2$ (left) and $d=3$ (right).

\begin{tabular}{ccc}
\hline$h_{0}$ & $(4.4 \mathrm{c}, \mathrm{e})$ & $(4.10 \mathrm{c}-\mathrm{e})$ \\
\hline $3.9018 \mathrm{e}-01$ & $3.9128 \mathrm{e}-02$ & $3.9394 \mathrm{e}-02$ \\
$1.9603 \mathrm{e}-01$ & $6.3381 \mathrm{e}-03$ & $6.3457 \mathrm{e}-03$ \\
$9.8135 \mathrm{e}-02$ & $2.1812 \mathrm{e}-02$ & $2.1788 \mathrm{e}-02$ \\
$4.9082 \mathrm{e}-02$ & $3.7650 \mathrm{e}-03$ & $3.7589 \mathrm{e}-03$ \\
$2.4543 \mathrm{e}-02$ & $2.2126 \mathrm{e}-03$ & $2.2088 \mathrm{e}-03$ \\
\hline
\end{tabular}

\begin{tabular}{ccc}
\hline$h_{0}$ & $(4.4 \mathrm{c}, \mathrm{e})$ & $(4.10 \mathrm{c}-\mathrm{e})$ \\
\hline $7.6537 \mathrm{e}-01$ & $5.3318 \mathrm{e}-02$ & $6.8256 \mathrm{e}-03$ \\
$4.0994 \mathrm{e}-01$ & $1.5836 \mathrm{e}-02$ & $1.6530 \mathrm{e}-02$ \\
$2.0854 \mathrm{e}-01$ & $9.5588 \mathrm{e}-03$ & $9.7333 \mathrm{e}-03$ \\
$1.0472 \mathrm{e}-01$ & $1.9850 \mathrm{e}-03$ & $2.0285 \mathrm{e}-03$ \\
$5.2416 \mathrm{e}-02$ & $2.1950 \mathrm{e}-03$ & $2.2494 \mathrm{e}-03$ \\
\hline
\end{tabular}

\subsection{Numerical simulations in $2 \mathrm{~d}$}

In this section we consider some numerical simulations for two-phase flow with insoluble surfactant in two space dimensions. We begin with a comparison between the schemes $\left(\mathrm{A}_{\mathrm{fd}}\right)$ and $\left(\mathrm{B}_{\mathrm{fd}}\right)$ for a rising bubble experiment that is motivated by the benchmark problems in [29] for two-phase Navier-Stokes flow.

\subsubsection{Rising bubble benchmark problem 1}

We use the setup described in [29], see Figure 2 there; i.e. $\Omega=(0,1) \times(0,2)$ with $\partial_{1} \Omega=[0,1] \times\{0,2\}$ and $\partial_{2} \Omega=\{0,1\} \times(0,2)$. Moreover, $\Gamma_{0}=\left\{\vec{z} \in \mathbb{R}^{2}:\left|\vec{z}-\left(\frac{1}{2}, \frac{1}{2}\right)^{T}\right|=\frac{1}{4}\right\}$. The physical parameters from the test case 1 in Table I of [29], in the absence of surfactant, are given by

$$
\rho_{+}=1000, \quad \rho_{-}=100, \quad \mu_{+}=10, \quad \mu_{-}=1, \quad \gamma_{0}=24.5, \quad \overrightarrow{f_{1}}=-0.98 \overrightarrow{e_{d}}, \quad \overrightarrow{f_{2}}=\overrightarrow{0},
$$

where, here and throughout, $\left\{\vec{e}_{j}\right\}_{j=1}^{d}$ denotes the standard basis in $\mathbb{R}^{d}$. The time interval chosen for the simulation is $[0, T]$ with $T=3$. For the surfactant problem we choose the parameters $\mathcal{D}_{\Gamma}=0.1$ and (2.13a) with $\beta=0.5$.

We start with a simulation for the scheme $\left(\mathrm{A}_{\mathrm{fd}}\right)$, i.e. $(4.4 \mathrm{a}-\mathrm{e})$, using the discretization parameters adapt ${ }_{7,3}$. The results can be seen on the left of Figure 2. Here two things should be noted. Firstly, the area of the inner phase has decreased in time. In fact, in this computation the relative area loss for the inner phase is $62 \%$. And secondly, we see that the vertices of the approximation $\Gamma^{m}$ are transported, similarly to the surfactant, with the fluid flow. This means that many vertices can be found at the bottom of the bubble, with hardly any vertices left at the top. The second behaviour can be improved by allowing local mesh refinements on $\Gamma^{m}$, recall Remark 4.5. In particular, we refine an element $\sigma^{m}$ on $\Gamma^{m}$ whenever $\mathcal{H}^{d-1}\left(\sigma^{m}\right)>\frac{7}{4} \max _{j=1, \ldots, J_{\Gamma}} \mathcal{H}^{d-1}\left(\sigma_{j}^{0}\right)$. Then the interface remains well resolved, and the final number of elements is $J_{\Gamma}^{M}=252>128=J_{\Gamma}^{0}$. However, coalescence of vertices can still be observed at the bottom of the bubble, see the plot on the right of Figure 2. 

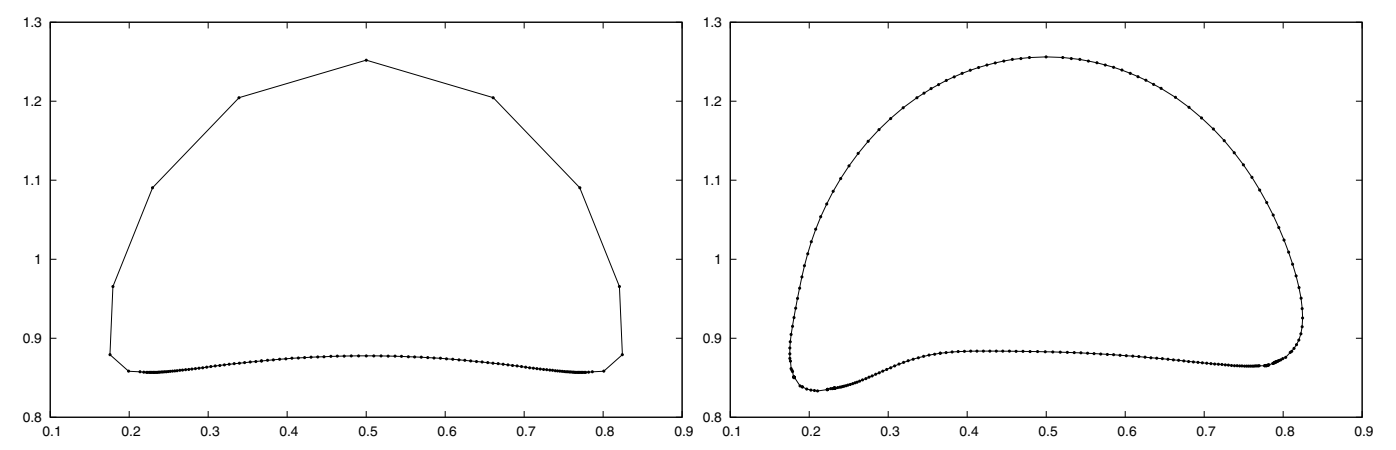

FiguRE 3. $\left(\operatorname{adapt}_{7,3}\right)$ Vertex distributions for the final bubbles for the benchmark problem 1 at time $T=3$ for a variant of the scheme $\left(\mathrm{A}_{\mathrm{fd}}\right)$ without local refinement on $\Gamma^{m}$ (left) and with local refinement (right). The loss of symmetry is caused by coalescence of vertices.
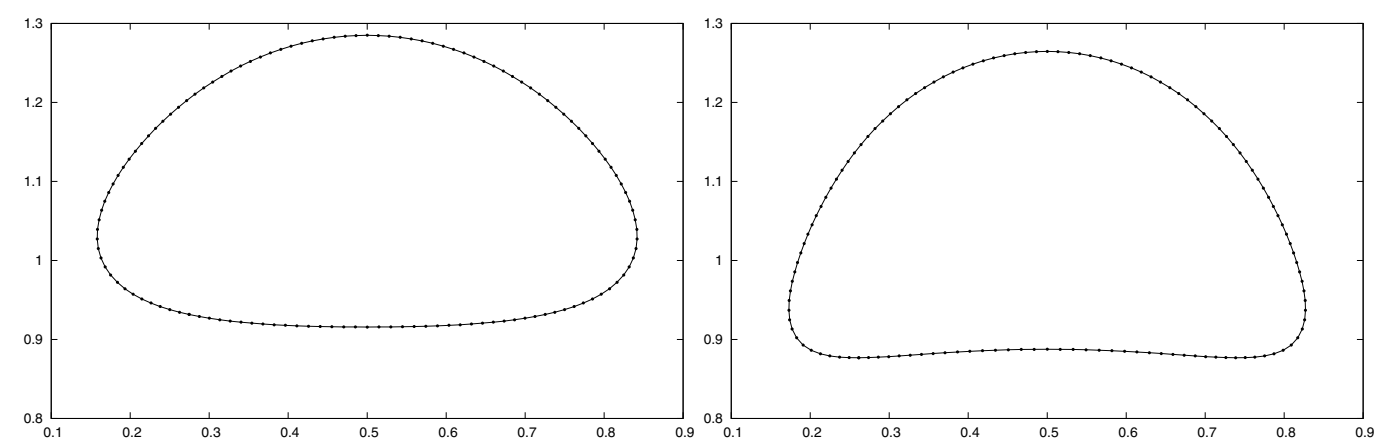

FiguRE 4. (adapt $t_{7,3}$ ) Vertex distributions for the final bubble for the benchmark problem 1 at time $T=3$ for the scheme $\left(\mathrm{B}_{\mathrm{fd}}\right)$. On the left the computation with $\beta=0$, on the right with $\beta=0.5$.

We remark that for the latter computation the area of the inner phase decreases by $14 \%$. For completeness we note that this dramatic area loss is connected to mass lumping being employed on the right hand side of (4.4c). To visualize this effect, we repeat the above computations now for $\left\langle\vec{U}^{m+1}, \vec{\chi}\right\rangle_{\Gamma^{m}}^{h}$ in (4.4c) replaced by $\left\langle\vec{U}^{m+1}, \vec{\chi}\right\rangle_{\Gamma^{m}}$. The semidiscrete variant of this new approximation then no longer satisfies the stability result in Theorem 3.3. However, in practice this approximation appears to perform much better, with the relative area loss of the inner phase now down to $1.4 \%$ for the simulation without local refinement. The simulation with local refinement leads to coalescence of vertices and a clear loss of symmetry, which is of course unphysical, see Figure 3.

The same computation for our preferred scheme $\left(\mathrm{B}_{\mathrm{fd}}\right)$, i.e. $(4.10 \mathrm{a}-\mathrm{e})$, where no local refinements need to be performed because the tangential movement of vertices yields an almost equidistributed approximation of $\Gamma^{\mathrm{m}}$, can be seen in Figure 4, where we compare the run with $\beta=0.5$ also to the case of constant surface tension, i.e. $\beta=0$. We remark that for these computations the areas of the two phases, as well as the total surfactant mass on $\Gamma^{m}$, were conserved.

In Figure 5 we show the surfactant concentrations $\Psi^{M}$ on the final bubble for the two schemes $\left(\mathrm{A}_{\mathrm{fd}}\right)$ and $\left(\mathrm{B}_{\mathrm{fd}}\right)$, where in the computation for the former scheme we employ local mesh refinements. We observe that the numerical results are in rough agreement, apart from the smaller bubble for the scheme $\left(\mathrm{A}_{\mathrm{fd}}\right)$ because of the loss of area for the inner phase. We also show a plot of the discrete surface energy $\left\langle F\left(\Psi^{m}\right), 1\right\rangle_{\Gamma^{m}}^{h}$, where for the scheme $\left(\mathrm{B}_{\mathrm{fd}}\right)$ it holds that $\left\langle F_{\varepsilon}\left(\Psi^{m}\right), 1\right\rangle_{\Gamma^{m}}^{h}=\left\langle F\left(\Psi^{m}\right), 1\right\rangle_{\Gamma^{m}}^{h}$ throughout the evolution. Here it can be seen 

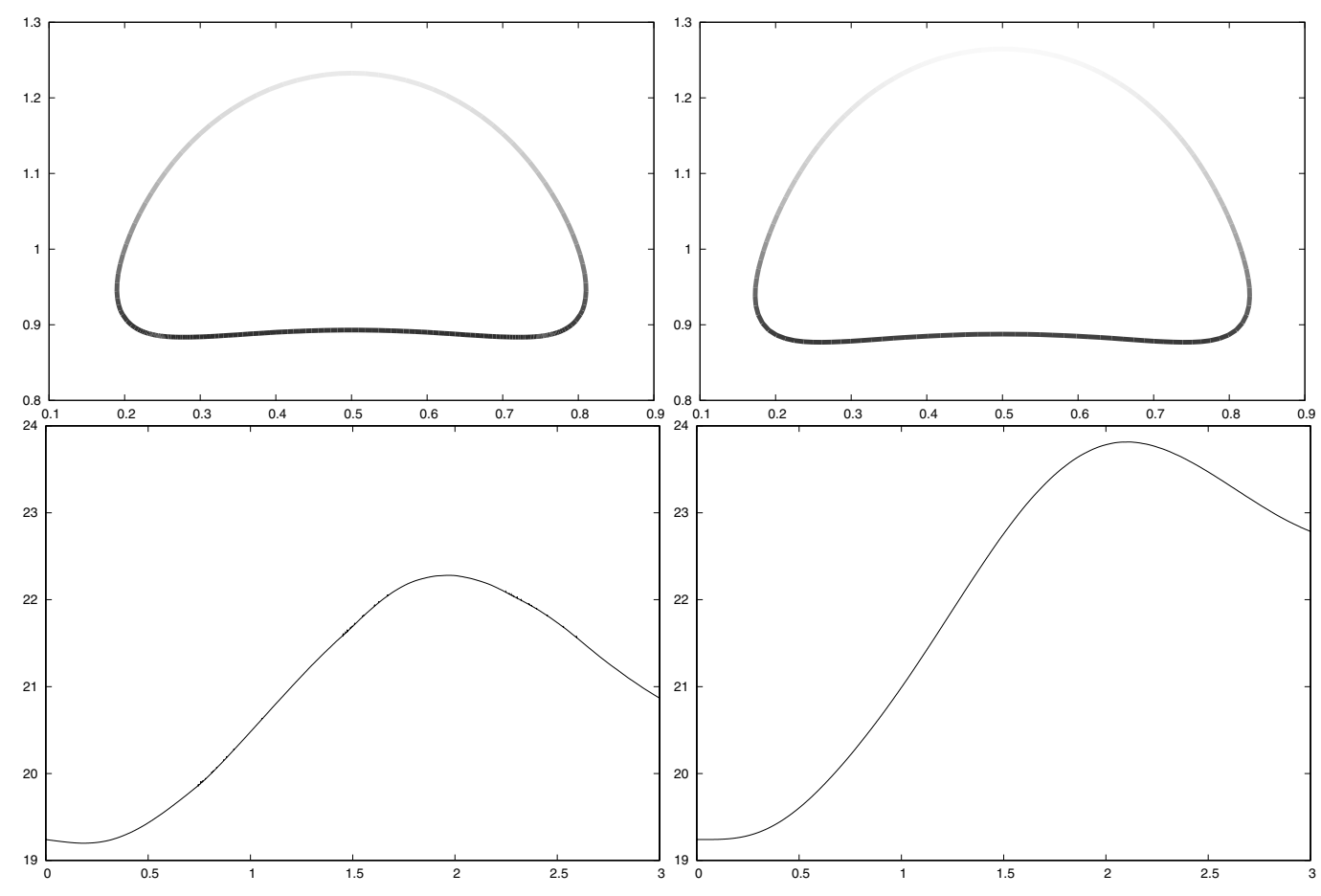

Figure 5. $\left(\operatorname{adapt}_{7,3}\right)$ The surfactant concentration on the final bubbles for the benchmark problem 1 at time $T=3$ for the schemes $\left(\mathrm{A}_{\mathrm{fd}}\right)$ and $\left(\mathrm{B}_{\mathrm{fd}}\right)$. The grey scales linearly with the surfactant concentration ranging from 0.4 (white) to 1.4 (black). Below we present plots of $\left\langle F\left(\Psi^{m}\right), 1\right\rangle_{\Gamma^{m}}^{h}$ over time for the two schemes.

that the plots for the two approximations differ significantly, most probably because of the area loss for the scheme $\left(\mathrm{A}_{\mathrm{fd}}\right)$.

The poor mesh properties of the scheme $\left(\mathrm{A}_{\mathrm{fd}}\right)$, together with the fact that the volume of the two phases is in general not conserved, mean that this scheme is not very practical. Of course, the same applies to the scheme from Remark 4.1. It is for this reason that from now on we only consider numerical experiments for the scheme $\left(\mathrm{B}_{\mathrm{fd}}\right)$.

The parameters in (5.1) were proposed in Table I of [29], in order to define a test case for two-phase flow, in the absence of surfactant, for which benchmark computations can be performed. We now report on these benchmark quantities also in the presence of surfactant. To this end, we recall from [3] our fully discrete approximations for the $x_{2}$-component of the bubble's centre of mass, the bubble's "degree of circularity" and the rise velocity:

$$
y_{c}^{m}=\frac{1}{\mathcal{L}^{2}\left(\Omega_{-}^{m}\right)} \int_{\Omega_{-}^{m}} x_{2} \mathrm{~d} \mathcal{L}^{2}, \quad \phi^{m}=2\left[\pi \mathcal{L}^{2}\left(\Omega_{-}^{m}\right)\right]^{\frac{1}{2}}\left[\mathcal{H}^{1}\left(\Gamma^{m}\right)\right]^{-1}, \quad V_{c}^{m}=\vec{v}^{m} \cdot \vec{e}_{d},
$$

where $\vec{v}^{m}=\left[\left(\rho_{-}^{m}, 1\right)\right]^{-1} \int_{\Omega} \rho_{-}^{m} \vec{U}^{m} \mathrm{~d} \mathcal{L}^{d}$, with $\rho_{-}^{m} \in S_{0}^{m}$ defined as in (4.2) but with $\rho_{+}$replaced by zero. Finally, we also define the relative overall area/volume loss as

$$
\mathcal{L}_{\text {loss }}=\frac{\mathcal{L}^{d}\left(\Omega_{-}^{0}\right)-\mathcal{L}^{d}\left(\Omega_{-}^{M}\right)}{\mathcal{L}^{d}\left(\Omega_{-}^{0}\right)}
$$

In Table 2 we report on these quantities for simulations with and without surfactant for our preferred scheme $\left(\mathrm{B}_{\mathrm{fd}}\right)$. Here we note that the numbers for the simulations without surfactant differ slightly from the ones in [3], 


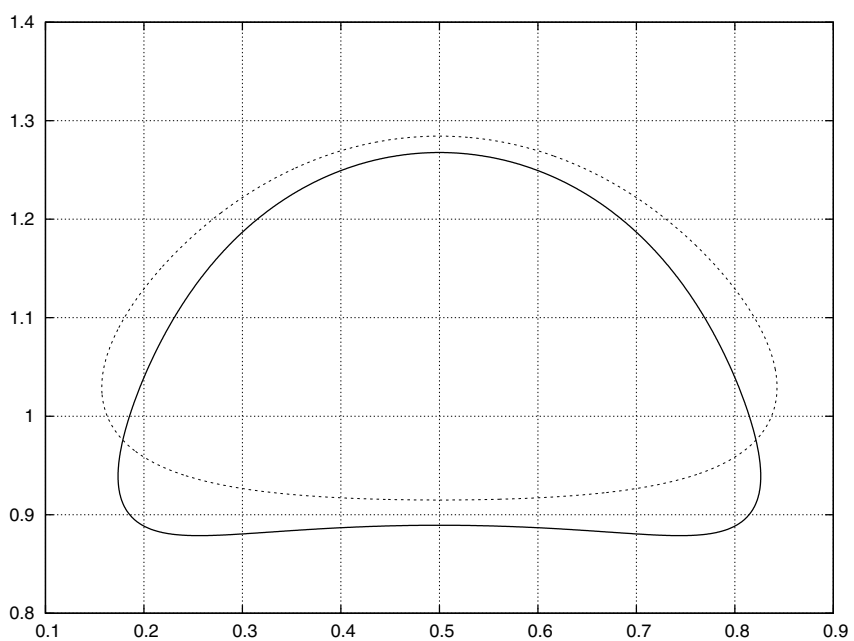

FiguRE 6. ( 5 adapt $_{11,5}$ ) The final bubble with surfactant for the benchmark problem 1 at time $T=3$. The clean bubble is shown dashed.

TABLE 2. Some quantitative results for the benchmark problem 1. Without surfactant (left) and with surfactant (right).

\begin{tabular}{ccccccccc}
\hline adapt $_{5,2}$ & adapt $_{7,3}$ & 2 adapt $_{9,4}$ & 5 adapt $_{11,5}$ & & adapt $_{5,2}$ & adapt $_{7,3}$ & 2 adapt $_{9,4}$ & 5 adapt $_{11,5}$ \\
\hline $0.0 \%$ & $0.0 \%$ & $0.0 \%$ & $0.0 \%$ & $\mathcal{L}_{\text {loss }}$ & $0.0 \%$ & $0.0 \%$ & $0.0 \%$ & $0.0 \%$ \\
0.9135 & 0.9069 & 0.9034 & 0.9022 & $\phi_{\text {min }}$ & 0.8779 & 0.8715 & 0.8681 & 0.8669 \\
2.0760 & 1.9420 & 1.9105 & 1.9028 & $t_{\phi=\phi_{\min }}$ & 2.1330 & 2.0710 & 2.0550 & 2.0504 \\
0.2477 & 0.2415 & 0.2413 & 0.2420 & $V_{c, \max }$ & 0.2279 & 0.2243 & 0.2236 & 0.2237 \\
0.9470 & 0.9360 & 0.9255 & 0.9698 & $t_{V_{c}=V_{c, \max }}$ & 1.0080 & 0.9040 & 0.9010 & 0.8916 \\
1.0906 & 1.0822 & 1.0814 & 1.0815 & $y_{c}(t=3)$ & 1.0423 & 1.0449 & 1.0467 & 1.0473 \\
\hline
\end{tabular}

Table 2, because the finite element approximations employed here is different, recall Remark 4.4. Nevertheless, there is still good agreement with the corresponding numbers from the finest discretization run of group 3 in [29], which are given by $0.9013,1.9000,0.2417,0.9239$ and 1.0817 . Here we note that of the three groups in [29], group 3 shows the most accurate and the most consistent results for the test case 1 . In what follows we present some visualizations of the numerical results for the runs with the discretization parameters 5 adapt 11,5 . A plot of $\Gamma^{M}$ can be seen in Figure 6, while the time evolution of the circularity, the centre of mass and the rise velocity are shown in Figures 7 and 8.

\subsubsection{Rising bubble benchmark problem 2}

In a second set of benchmark computations, we fix

$$
\rho_{+}=1000, \quad \rho_{-}=1, \quad \mu_{+}=10, \quad \mu_{-}=0.1, \quad \gamma_{0}=1.96, \quad \overrightarrow{f_{1}}=-0.98 \overrightarrow{e_{d}}, \quad \overrightarrow{f_{2}}=\overrightarrow{0},
$$

as in test case 2 in Table I of [29]. For the surfactant problem we again let $\mathcal{D}_{\Gamma}=0.1$ and let $\beta=0.5$ in (2.13a). In Table 3 we report on some benchmark quantities for simulations with and without surfactant for our preferred scheme $\left(\mathrm{B}_{\mathrm{fd}}\right)$. Here we note that in contrast to the experiments in Section 5.2.1, there is little difference between the numbers for the runs with and without surfactant. This is because in the simulations for (5.3) the large values of $\frac{\rho_{+}}{\rho_{-}}$and $\frac{\mu_{+}}{\mu_{-}}$dominate the evolution. In particular, they lead to elongated fingers developing at the bottom of the rising bubble which means that there is a significant growth in the overall interface length. In order to account for this growth, we locally refine $\Gamma^{m}$ in all the simulations for the parameters 


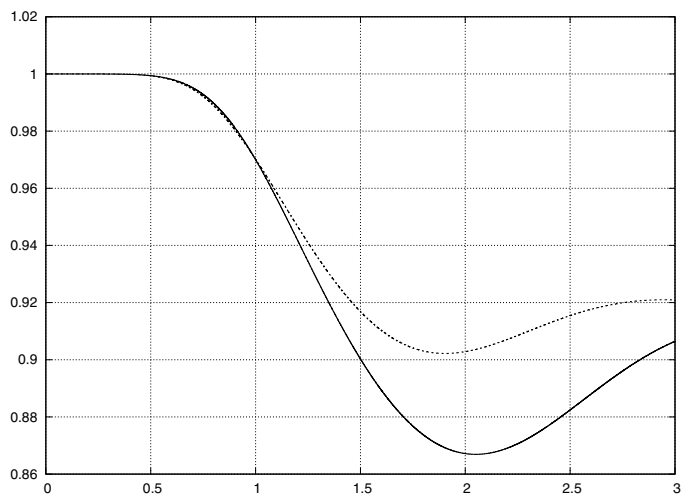

Figure 7. (5 adapt 11,5 ) Circularity of the surfactant bubble for the benchmark problem 1 . The dashed line is for the clean bubble.
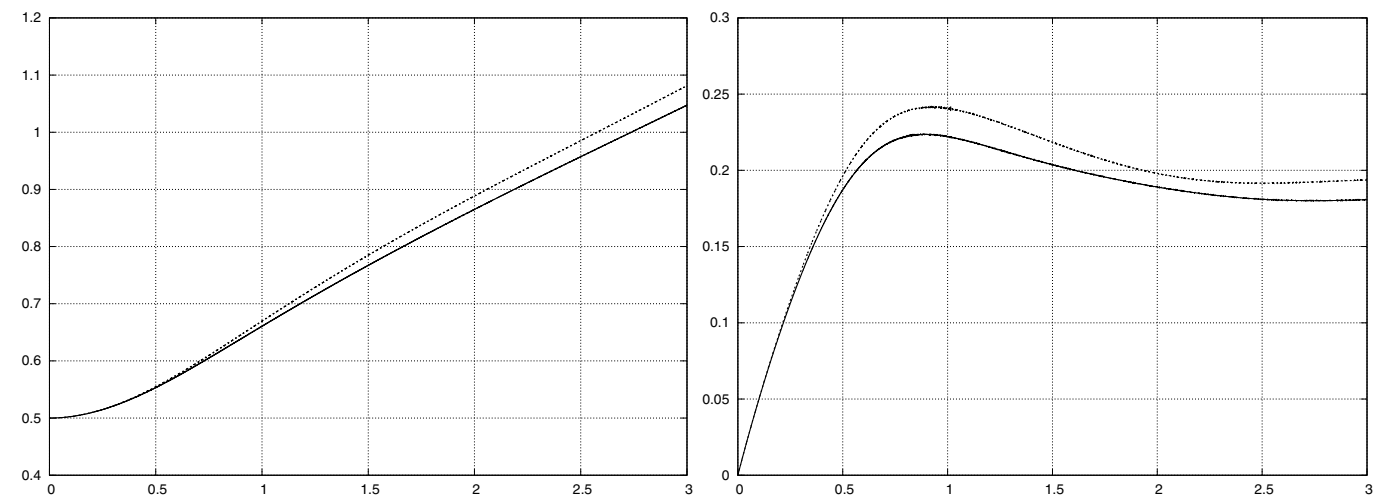

Figure 8. ( 5 adapt $\left._{11,5}\right)$ Centre of mass and rise velocity for the surfactant bubble for the benchmark problem 1 . The dashed lines are for the clean bubble.

as in (5.3). Here, similarly to the experiment on the right of Figure 2, we refine an element $\sigma^{m}$ on $\Gamma^{m}$ whenever $\mathcal{H}^{d-1}\left(\sigma^{m}\right)>\frac{7}{4} \max _{j=1, \ldots, J_{\Gamma}} \mathcal{H}^{d-1}\left(\sigma_{j}^{0}\right)$. For completeness we note that the corresponding numbers from the finest discretization run of group 3 in [29], in the absence of surfactant, are given by $0.5144,3.0000,0.2502$, $0.7317,0.2393,2.0600$ and 1.1376 . In what follows we present some visualizations of the numerical results for the runs with the discretization parameters 2 adapt $_{9,4}$. A plot of $\Gamma^{M}$ can be seen in Figure 9, where we also show the final surfactant concentration $\Psi^{M}$. Here we observe that most of the surfactant has accumulated at the inner side walls of the lower part of the bubble. It is worth pointing out that our numerical method has no difficulties in computing the evolution of the advection-diffusion equation on a highly deformed interface as seen in Figure 9. As the surfactant in this experiment has hardly any effect on the circularity, the centre of mass and the rise velocity of the bubble, we omit detailed plots for these quantities here. But the same plots for the clean bubble can be found in [3].

\subsubsection{Rising circular bubble}

In the rising bubble simulations in the two previous subsections, the rise velocity of the bubble was not dramatically altered by adding surfactant onto the interface. However, a lower rise velocity is often observed for contaminated bubbles in experiments, see e.g. [15], and we want to highlight this effect in the next numerical simulation. 

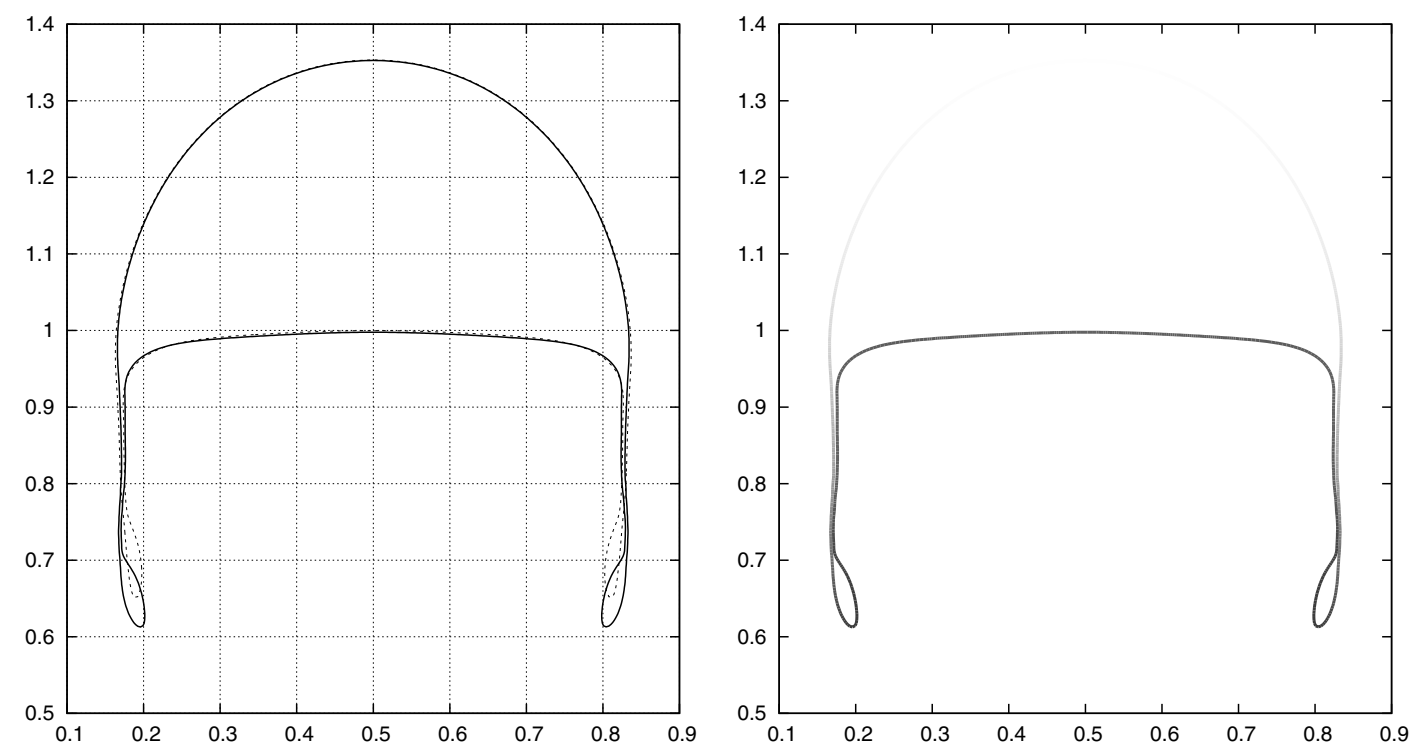

Figure 9. $\left(2\right.$ adapt $\left._{9,4}\right)$ The final bubble with surfactant for the benchmark problem 2 at time $T=3$, with the surfactant concentration on the right. The grey scales linearly with the surfactant concentration ranging from 0.1 (white) to 0.9 (black). The dashed curve on the left represents the final shape of the clean bubble.

TABLE 3. Some quantitative results for the benchmark problem 2. Without surfactant (left) and with surfactant (right).

\begin{tabular}{ccccccc}
\hline adapt $_{5,2}$ & adapt $_{7,3}$ & 2 adapt $_{9,4}$ & & adapt $_{5,2}$ & adapt $_{7,3}$ & 2 $_{\text {adapt }}, 4$ \\
\hline $0.0 \%$ & $0.0 \%$ & $0.0 \%$ & $\mathcal{L}_{\text {loss }}$ & $0.0 \%$ & $0.0 \%$ & $0.0 \%$ \\
0.5890 & 0.5198 & 0.5165 & $\phi_{\min }$ & 0.5449 & 0.4998 & 0.4891 \\
3.0000 & 3.0000 & 3.0000 & $t_{\phi}=\phi_{\min }$ & 3.0000 & 3.0000 & 3.0000 \\
0.2584 & 0.2480 & 0.2489 & $V_{c, \max 1}$ & 0.2563 & 0.2465 & 0.2476 \\
0.8800 & 0.7610 & 0.7295 & $t_{V_{c}=V_{c, \max 1}}$ & 0.8830 & 0.7340 & 0.7390 \\
0.2283 & 0.2305 & 0.2357 & $V_{c, \max 2}$ & 0.2283 & 0.2326 & 0.2391 \\
2.0000 & 1.9510 & 2.0485 & $t_{V_{c}}=V_{c, \max 2}$ & 2.2550 & 2.0330 & 2.0825 \\
1.1275 & 1.1239 & 1.1319 & $y_{c}(t=3)$ & 1.1217 & 1.1197 & 1.1294 \\
\hline
\end{tabular}

We consider the same domain $\Omega=(0,1) \times(0,2)$ as in Section 5.2.1, with the same boundary conditions as there. As before, we let $\Gamma_{0}=\left\{\vec{z} \in \mathbb{R}^{2}:\left|\vec{z}-\left(\frac{1}{2}, \frac{1}{2}\right)^{T}\right|=\frac{1}{4}\right\}$. The physical parameters, which we choose as in ([33], Fig. 16), are given by

$$
\rho_{+}=1, \quad \rho_{-}=0.1, \quad \mu_{+}=\frac{1}{2}, \quad \mu_{-}=\frac{1}{80}, \quad \gamma_{0}=1, \quad \overrightarrow{f_{1}}=-\overrightarrow{e_{d}}, \quad \overrightarrow{f_{2}}=\overrightarrow{0},
$$

with the time interval defined by $[0, T]$ with $T=30$. For the surfactant problem we choose the parameters $\mathcal{D}_{\Gamma}=0.01$ and (2.13a) with $\beta=0.5$. Overall this experiment corresponds to an insoluble surfactant variant of the simulation presented in ([33], Fig. 16), where a soluble surfactant is considered. To demonstrate the effect that adding surfactant to the bubble has on the velocity profile inside the bubble, in Figure 10 we plot the relative velocity $\vec{U}^{M}-\vec{v}^{M}$, recall (5.2), for the two cases of clean and contaminated bubble. Clearly, the two vortices inside the clean bubble almost vanish completely when surfactant is added. This reduces the rise velocity significantly, as can be seen at the bottom of Figure 10. 

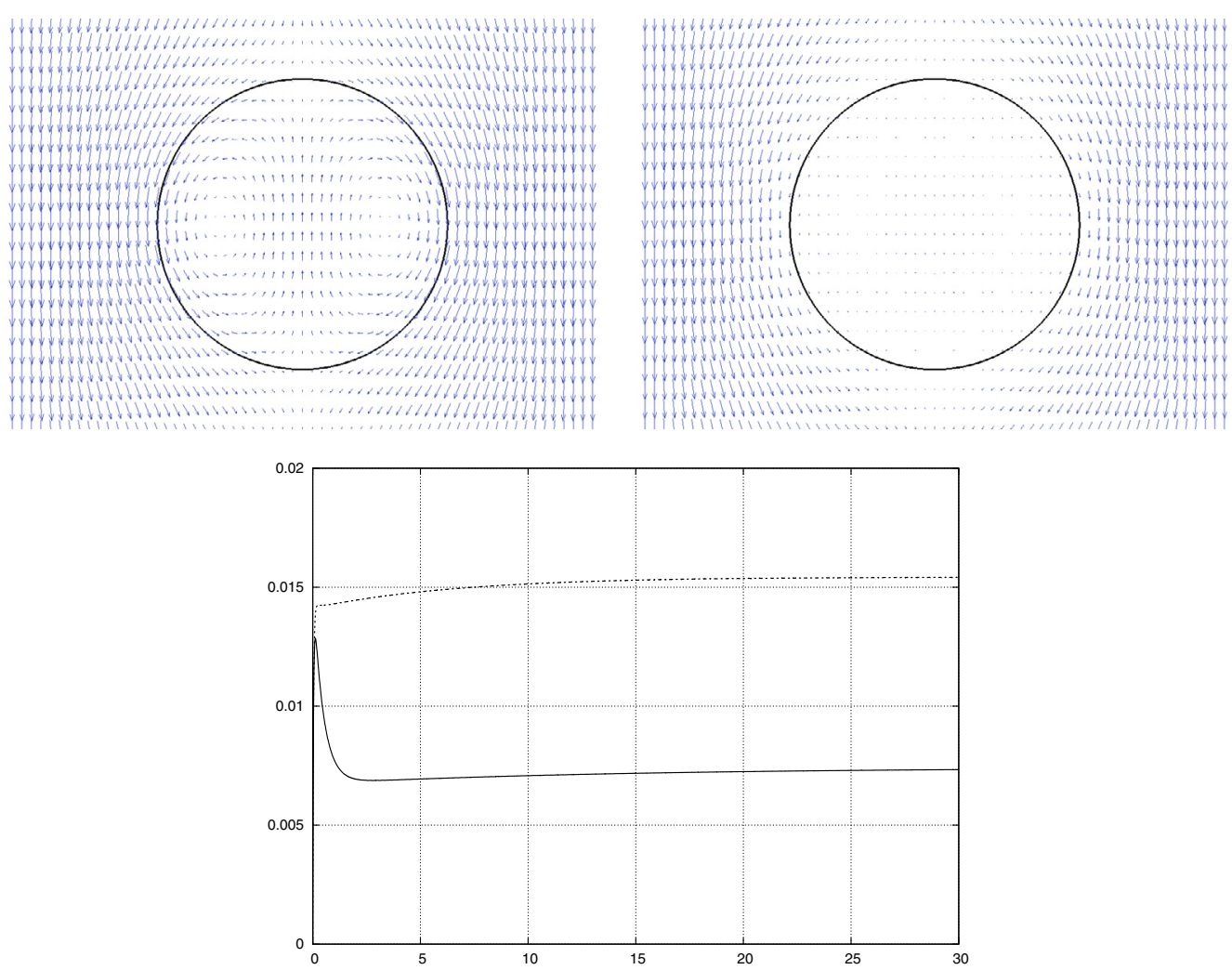

FiguRE 10. (adapt ${ }_{9,4}$ ) Relative velocity vectors $\vec{U}^{M}-\vec{v}^{M}$ for the final bubble without surfactant (left) and with surfactant (right) at time $T=30$. Below a comparison of the rise velocities for the two bubbles, where the dashed line is for the clean bubble.

\subsubsection{Bubble in shear flow}

In the literature on numerical methods for two-phase flow with insoluble surfactant it is often common to consider shear flow experiments for an initially circular bubble in order to study the effect of surfactants and of different equations of state. In this subsection, we will perform such simulations for our preferred scheme $\left(\mathrm{B}_{\mathrm{fd}}\right)$. Here we consider the setup from ([32], Fig. 1). In particular, we let $\Omega=(-5,5) \times(-2,2)$ and prescribe the inhomogeneous Dirichlet boundary condition $\vec{g}(\vec{z})=\left(\frac{1}{2} z_{2}, 0\right)^{T}$ on $\partial \Omega=\partial_{1} \Omega$. Moreover, $\Gamma_{0}=\left\{\vec{z} \in \mathbb{R}^{2}:|\vec{z}|=1\right\}$. The physical parameters are given by

$$
\rho_{+}=\rho_{-}=1, \quad \mu_{+}=\mu_{-}=0.1, \quad \gamma_{0}=0.2, \quad \mathcal{D}_{\Gamma}=0.1, \quad \vec{f}=\overrightarrow{0}, \quad \overrightarrow{u_{0}}=\vec{g} .
$$

First we compare the evolutions for the linear equation of state (2.13a) for (i) $\beta=0$, (ii) $\beta=0.25$ and (iii) $\beta=0.5$. Our numerical results appear to agree very well with the ones in Figure 1 of [32]; see Figure 11 for more details. On recalling (5.2), we note that the "circularities" $\phi^{M}$ of the final bubbles are given by $0.68,0.59$ and 0.51, respectively. Moreover, we remark that for these simulations the relative overall area loss satisfies $\left|\mathcal{L}_{\text {loss }}\right|<0.02 \%$, and the same holds true for all of the remaining numerical experiments in this subsection.

In the next experiment we choose the nonlinear equation of state (2.13b) with $\psi_{\infty}=\frac{1}{\beta}$ (see also [32], Fig. 6). We show the evolutions of the drop for $\beta=0.25$ and for $\beta=0.5$ in Figure 12. A detailed comparison of the final drop shapes for the two equations of state $(2.13 \mathrm{a}, \mathrm{b})$ can be seen in Figure 13. As expected, the difference between the simulations for the two equations of state are more pronounced for the larger value of $\beta$. 

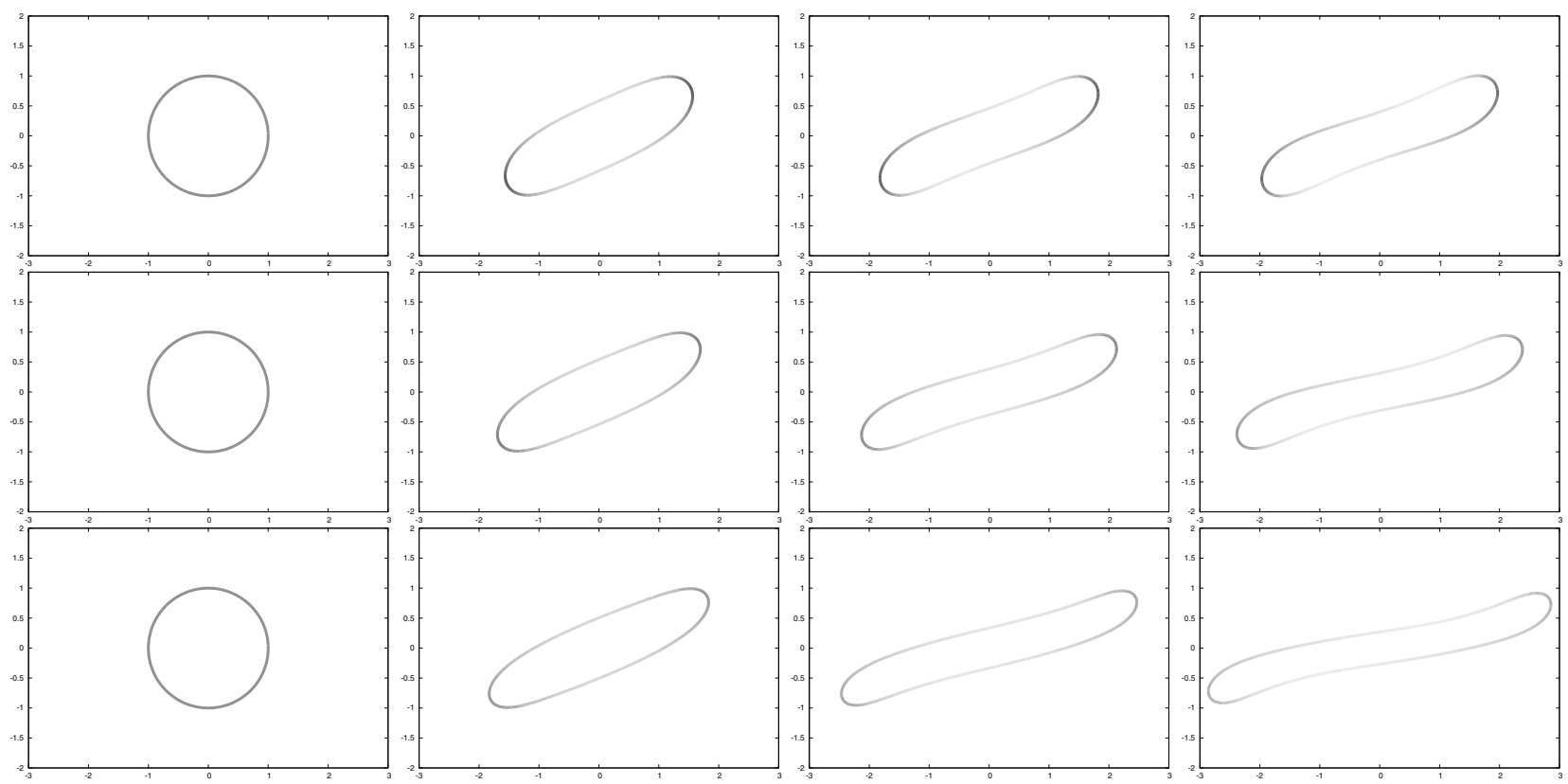

Figure 11. (2 adapt $\left._{9,4}\right)$ The time evolution of a drop in shear flow for $(2.13 \mathrm{a})$ with $\beta=0$ (top), $\beta=0.25$ (middle) and $\beta=0.5$ (bottom). Plots are at times $t=0,4,8,12$. The grey scales linearly with the surfactant concentration ranging from 0.2 (white) to 1.6 (black).
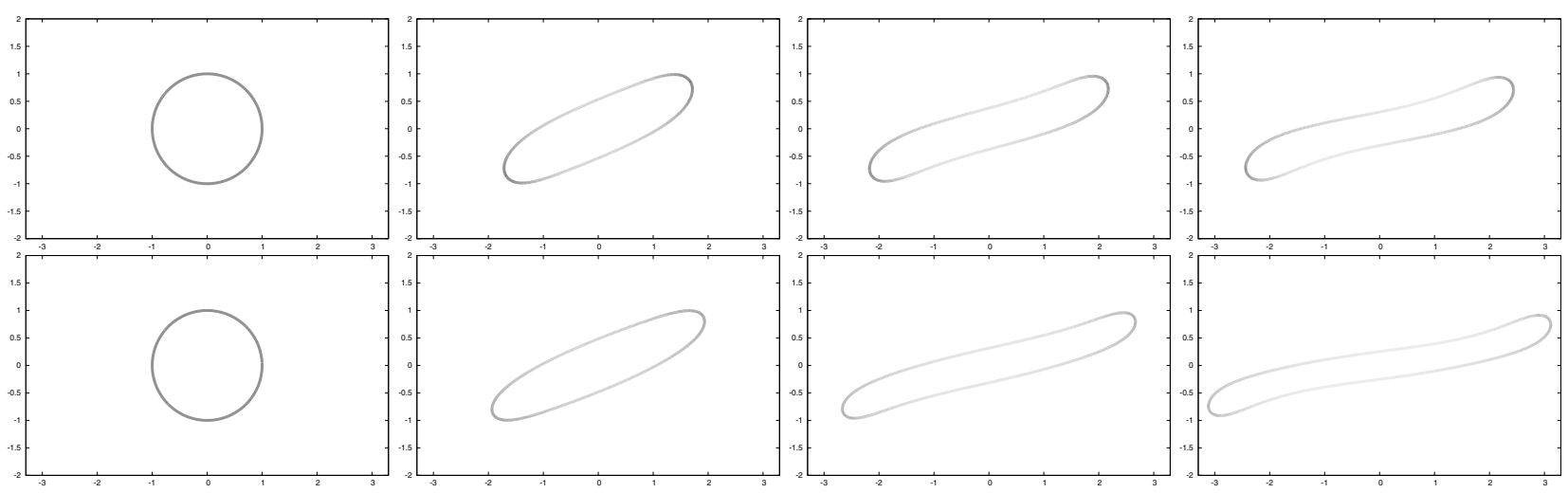

Figure 12. (2 adapt $\left._{9,4}\right)$ The time evolution of a drop in shear flow for $(2.13 \mathrm{~b})$ with $\beta=0.25$ (top) and $\beta=0.5$ (bottom). Plots are at times $t=0,4,8,12$. The grey scales linearly with the surfactant concentration ranging from 0.2 (white) to 1.6 (black).

In Figure 14 we compare the previously used (2.13a) with $\beta=0.5$ and (2.13b) with $\psi_{\infty}^{-1}=\beta=0.5$ to (2.13b) with $\beta=0.5$ and $\psi_{\infty}=1.3$. This indicates that the initial drop should now be more unstable. However, the evolution is not very different to what we saw before, see Figure 15. This is despite the maximum discrete surfactant concentration being $\approx 1.08$, which means that the discrete surface tension $\gamma\left(\Psi^{m}\right)$ at times is negative. In fact, the observed minimum value is $<-0.03$, compare with Figure 14, but this posed no problem for our numerical method. 

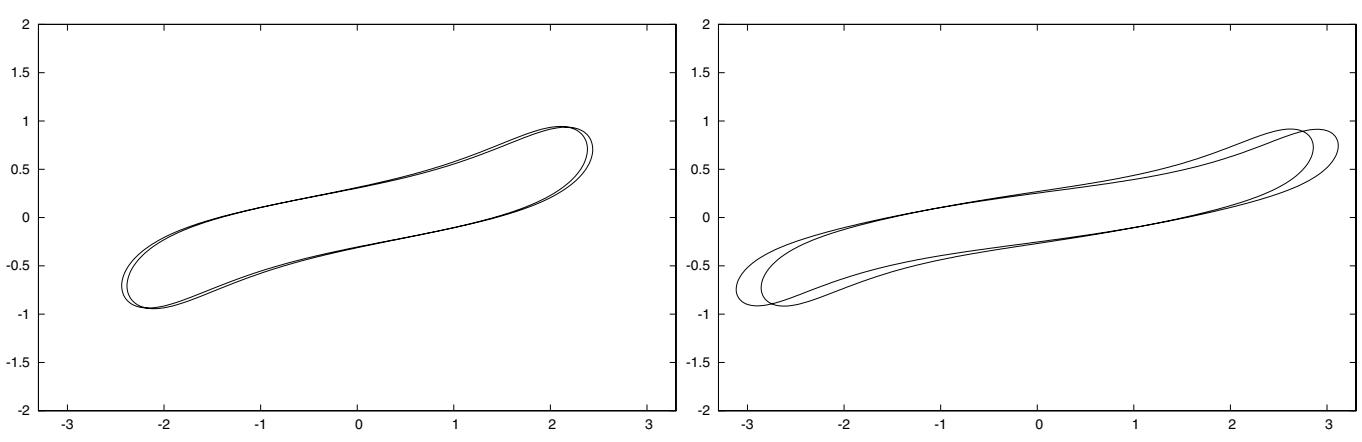

Figure 13. (2 adapt $_{9,4}$ ) Comparison of the final drop shapes in shear flow for a linear (2.13a) and a nonlinear (2.13b) equation of state with $\beta=0.25$ (left) and $\beta=0.5$ (right). In each case the shape for $(2.13 \mathrm{~b})$ is more elongated.

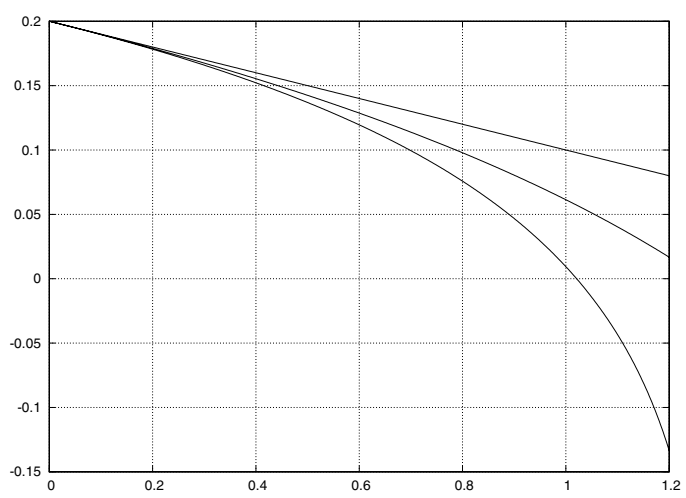

Figure 14. $(\beta=0.5)$ Plots of $\gamma(r)$ for the linear equation of state (2.13a) and the nonlinear equation of state $(2.13 \mathrm{~b})$ with $\psi_{\infty}=2$ and 1.3 .
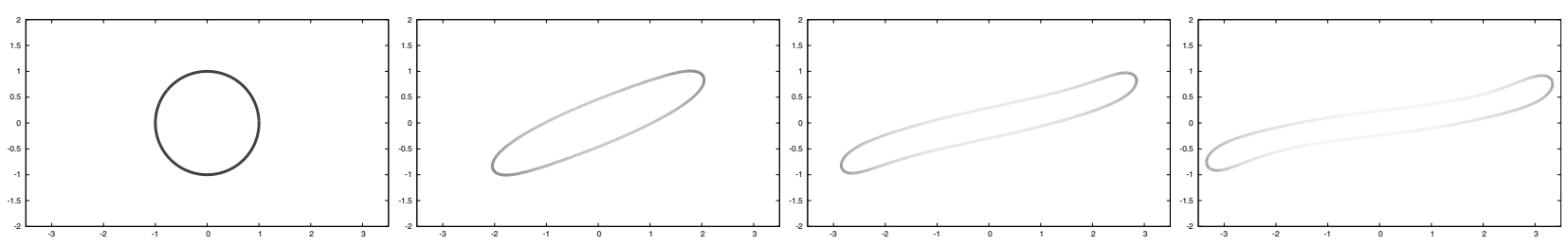

Figure 15. $\left(2\right.$ adapt $\left._{9,4}\right)$ The time evolution of a drop in shear flow for $(2.13 \mathrm{~b})$ with $\beta=0.5$ and $\psi_{\infty}=1.3$. Plots are at times $t=0,4,8,12$. The grey scales linearly with the surfactant concentration ranging from 0.3 (white) to 1.1 (black).

On returning back to the linear equation of state (2.13a), we also present a numerical simulation for different densities and viscosities. In particular, we leave all the parameters as in (5.4), but now choose

$$
\rho_{+}=10, \quad \rho_{-}=1, \quad \mu_{+}=1, \quad \mu_{-}=0.1 .
$$

We show the evolution of the drop in Figure 16 for $\beta=0,0.25$ and 0.5 . In contrast to Figure 11, the presence of surfactant has very little impact on the shape of the drop here. However, the interfaces in Figure 16 are more distorted and have higher curvatures at the ends, which is a well-known fact when the viscosity of the drop is much less than the one of the surrounding fluid, see [35]. 

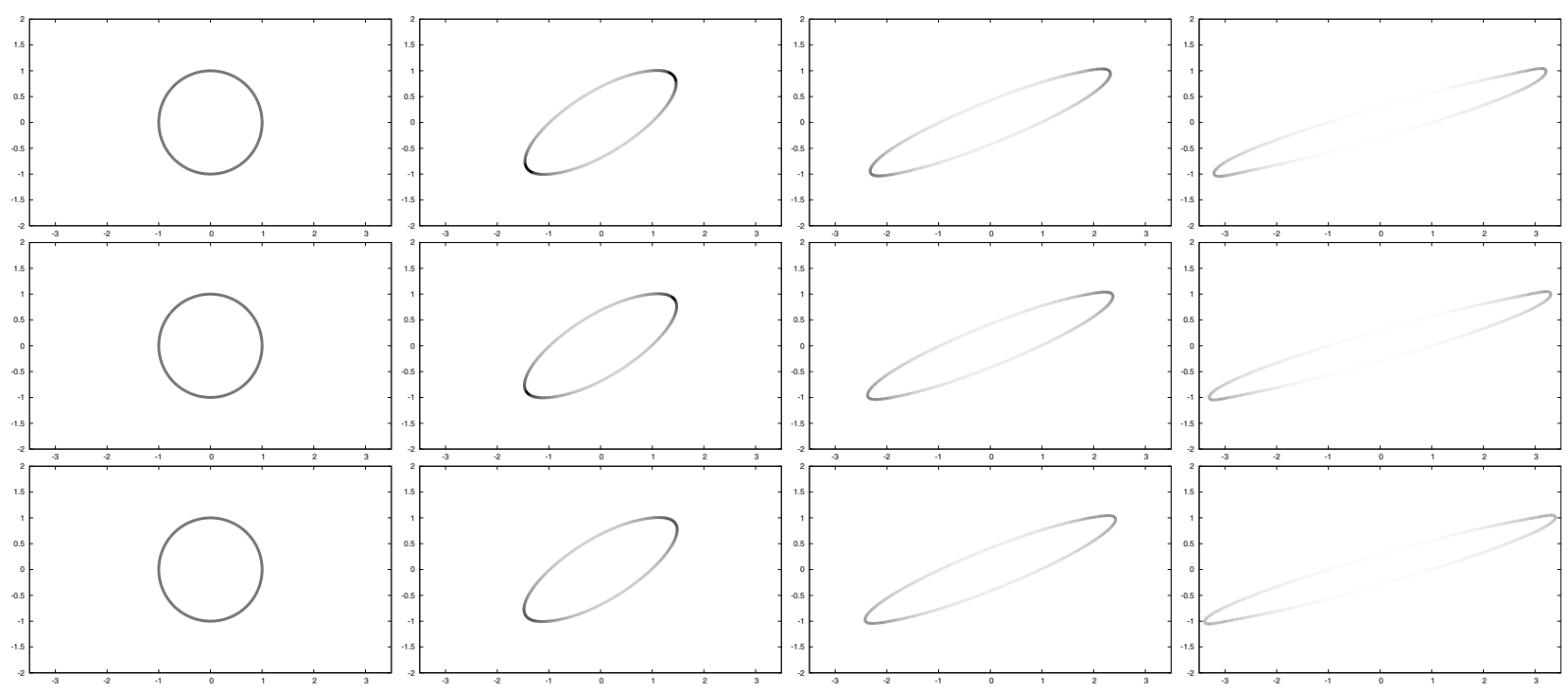

FiguRE 16. (2 adapt $\left._{9,4}\right)$ The time evolution of a drop in shear flow for $(2.13 \mathrm{a})$ with $\beta=0$ (top), $\beta=0.25$ (middle) and $\beta=0.5$ (bottom). Here $\rho_{+}=10, \rho_{-}=1, \mu_{+}=1, \mu_{-}=0.1$. Plots are at times $t=0,2,4,6$. The grey scales linearly with the surfactant concentration ranging from 0.3 (white) to 1.3 (black).

TABLE 4. Some quantitative results for the $3 \mathrm{~d}$ benchmark problem 1 . Without surfactant (left) and with surfactant (right).

\begin{tabular}{ccccc}
\hline adapt $_{5,2}$ & adapt $_{6,3}$ & & adapt $_{5,2}$ & adapt $_{6,3}$ \\
\hline $0.0 \%$ & $0.0 \%$ & $\mathcal{L}_{\text {loss }}$ & $0.0 \%$ & $0.0 \%$ \\
0.9570 & 0.9508 & $\phi_{\text {min }}$ & 0.9347 & 0.9297 \\
3.0000 & 3.0000 & $t_{\phi=\phi_{\min }}$ & 2.8300 & 2.9970 \\
0.3822 & 0.3845 & $V_{c, \max }$ & 0.3252 & 0.3296 \\
1.1930 & 1.0790 & $t_{V_{c}=V_{c, \max }}$ & 1.1230 & 0.8960 \\
1.5515 & 1.5555 & $z_{c}(t=3)$ & 1.3807 & 1.3902 \\
\hline
\end{tabular}

\subsection{Numerical simulations in $3 d$}

In this section we consider some numerical simulations for two-phase flow with insoluble surfactant in three space dimensions. Here we will always report on simulations for our preferred scheme $\left(\mathrm{B}_{\mathrm{fd}}\right)$.

\subsubsection{Rising bubble benchmark problem 1}

Here we consider the natural $3 \mathrm{~d}$ analogue of the problem in Section 5.2.1. To this end, we let $\Omega=(0,1) \times$ $(0,1) \times(0.2)$ with $\partial_{1} \Omega=[0,1] \times[0,1] \times\{0,2\}$ and $\partial_{2} \Omega=\partial \Omega \backslash \partial_{1} \Omega$. Moreover, we set $T=3, \Gamma_{0}=\left\{\vec{z} \in \mathbb{R}^{3}\right.$ : $\left.\left|\vec{z}-\left(\frac{1}{2}, \frac{1}{2}, \frac{1}{2}\right)^{T}\right|=\frac{1}{4}\right\}$, and choose the physical parameters as in (5.1). The time interval chosen for the simulation is again $[0, T]$ with $T=3$. For the surfactant problem we choose the parameters $\mathcal{D}_{\Gamma}=0.1$ and (2.13a) with $\beta=0.5$.

Some quantitative values for the evolution are given in Table 4, where we have introduced the natural extensions of the quantities defined in (5.2). In particular, the discrete approximations of the $x_{3}$-component of 

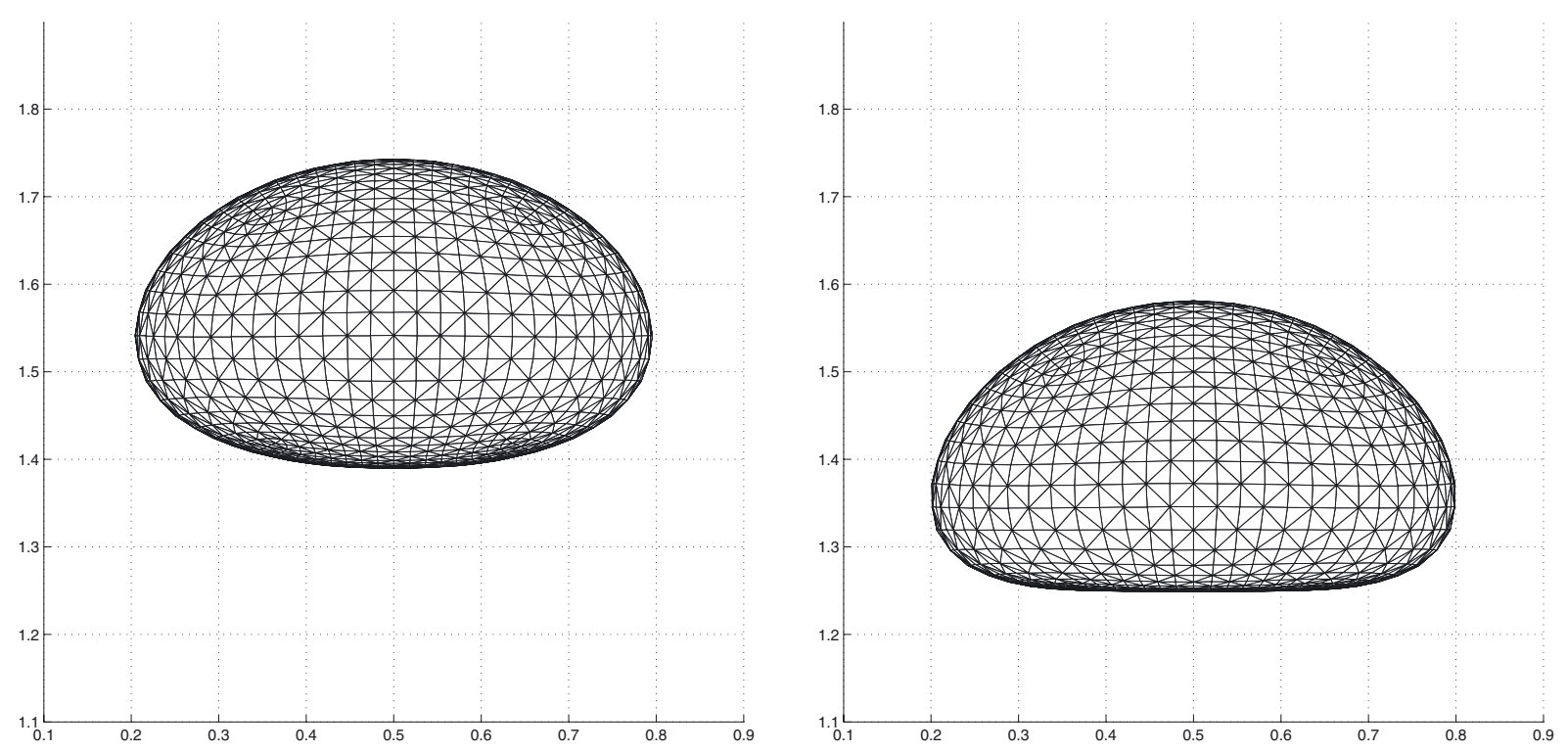

FiguRE 17. (adapt ${ }_{6,3}$ ) Side view of the final bubble for the $3 \mathrm{~d}$ benchmark problem 1 at time $T=3$. Without surfactant (left) and with surfactant (right).
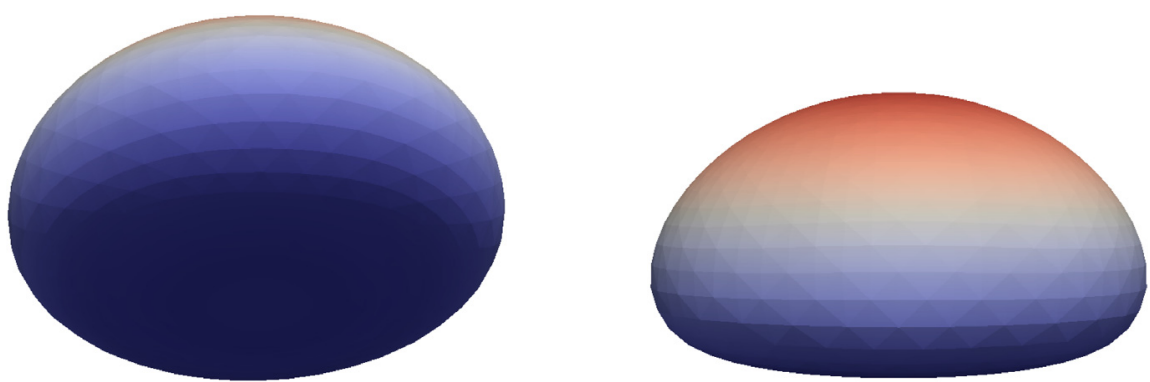

FiguRE 18. $\left(\right.$ adapt $_{6,3}$ ) The final surfactant concentration $\Psi^{M}$ on $\Gamma^{M}$. Here the colour ranges from red (0.5) to blue (1.2). (In color online).

the bubble's centre of mass and the "degree of sphericity" are defined by

$$
z_{c}^{m}=\frac{1}{\mathcal{L}^{3}\left(\Omega_{-}^{m}\right)} \int_{\Omega_{-}^{m}} x_{3} \mathrm{~d} \mathcal{L}^{3}, \quad \phi^{m}=\pi^{\frac{1}{3}}\left[6 \mathcal{L}^{3}\left(\Omega_{-}^{m}\right)\right]^{\frac{2}{3}}\left[\mathcal{H}^{2}\left(\Gamma^{m}\right)\right]^{-1} .
$$

In what follows we present some visualizations of the numerical results for the runs with adapt 6,3 . A comparison of the final meshes for the runs with and without surfactant can be seen in Figure 17, while the discrete surfactant concentration for the run with surfactant can be seen in Figure 18.

\subsubsection{Bubble in shear flow}

In this subsection we report on the $3 \mathrm{~d}$ analogues of the computations shown in Figure 11. In particular, in Figure 19 we show shear flow experiments on the domain $\Omega=(-5,5) \times(-2,2)^{2}$ with $\partial \Omega=\partial_{1} \Omega$ and $\vec{g}(\vec{z})=\left(\frac{1}{2} z_{3}, 0,0\right)^{T}$. The physical parameters are as in (5.4), and we compare the evolutions for the linear equation of state (2.13a) for (i) $\beta=0$; (ii) $\beta=0.25$; and (iii) $\beta=0.5$. As the discretization parameters we choose adapt ${ }_{5,2}^{\star}$, which are the same as for adapt ${ }_{5,2}$, apart from $\tau=0.01$ and $\left(K_{\Gamma}, J_{\Gamma}\right)=(1538,3072)$, i.e. 

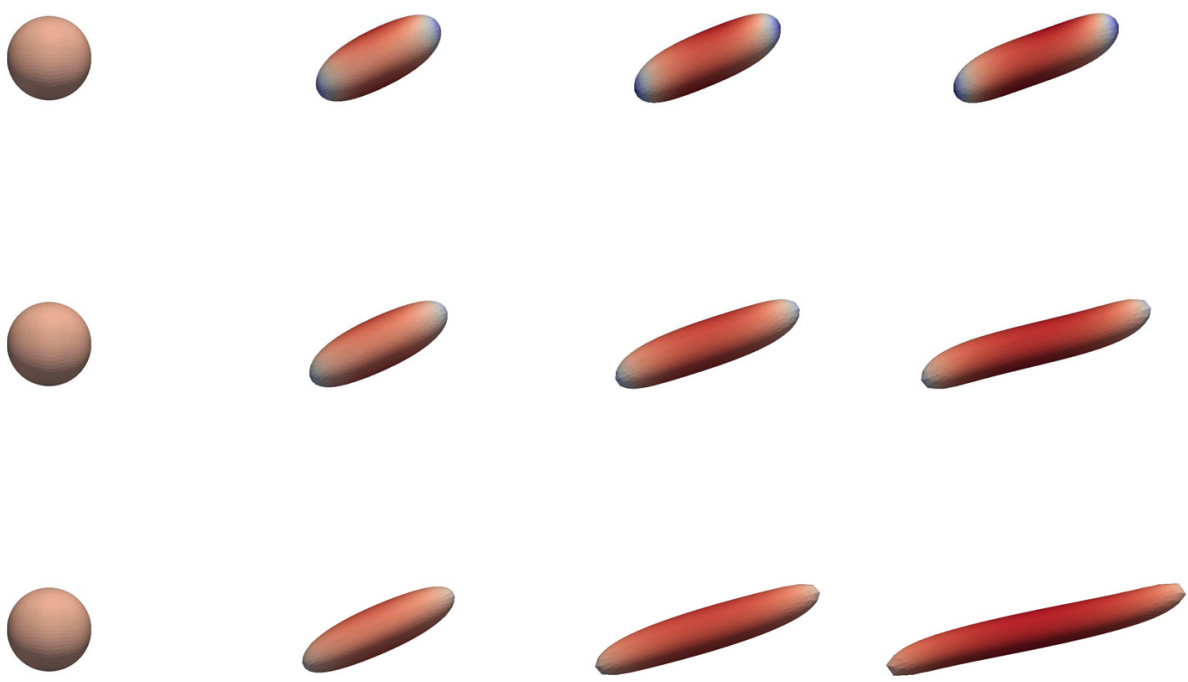

FiguRE 19. $\left(\operatorname{adapt}_{5,2}^{\star}\right)$ The discrete surfactant concentrations $\Psi^{m}$ at times $t=0,4,8,12$ for $\beta=0$ (top), $\beta=0.25$ (middle) and $\beta=0.5$ (bottom). The colour ranges from red (0.5) to blue (1.9). (In color online).

$\operatorname{adapt}_{5,2}^{\star}$ uses a larger time step size and a finer interface mesh compared to adapt ${ }_{5,2}$. Our three dimensional results turn out to be very similar to the two dimensional results in Figure 11; see Figure 19 for more details.

\section{Appendix A. Exact solution for the ADVECtion Diffusion EQUATION}

Following [19], Example 7.3, we present a true solution to the inhomogeneous advection diffusion equation

$$
\partial_{t}^{\bullet} \psi+\psi \nabla_{s} \cdot \vec{u}-\Delta_{s} \psi=f_{\Gamma} \quad \text { on } \Gamma(t),
$$

recall (2.8), in a situation where the fluid velocity $\vec{u}$, and hence the evolution of $\Gamma(t)$, is given. The surface is given by $\Gamma(t)=\left\{\vec{z} \in \mathbb{R}^{d}: \phi(\vec{z}, t)=1\right\}$, where

$$
\phi(\vec{z}, t)=[a(t)]^{-1} z_{1}^{2}+\sum_{i=2}^{d} z_{i}^{2},
$$

so that the moving surface $\Gamma(t)$ is an ellipsoid with time dependent $x_{1}$-axis. Here we choose $a(t)=1+\sin (\pi t)$, and as the parameterization $\vec{x}(\cdot, t): \mathbb{S}^{d-1} \rightarrow \Gamma(t)$, where $\mathbb{S}^{d-1}:=\left\{\vec{q} \in \mathbb{R}^{d}:|\vec{q}|=1\right\}$, we choose

$$
\vec{x}(\vec{q}, t)=[a(t)]^{\frac{1}{2}} q_{1} \vec{e}_{1}+\sum_{i=2}^{d} q_{i} \vec{e}_{i} \quad \forall \vec{q} \in \mathbb{S}^{d-1}, \quad t \in \mathbb{R}_{\geq 0} .
$$

On recalling (2.1), for the fluid velocity we naturally choose

$$
\vec{u}(\vec{z}, t)=\frac{1}{2}[a(t)]^{-1} a^{\prime}(t) z_{1} \vec{e}_{1} \quad \vec{z} \in \Omega,
$$

so that $\left.\vec{u}(\vec{z}, t)\right|_{\Gamma(t)}=\overrightarrow{\mathcal{V}}(\vec{z}, t)$ for $\vec{z} \in \Gamma(t)$. As an exact solution we choose $\psi(\vec{z}, t)=\mathrm{e}^{-6 t} z_{1} z_{2}$, and hence it remains to calculate the right hand side $f_{\Gamma}$ in (A.1) for our chosen $\psi$ and $\vec{u}$. To this end we note that

$$
f_{\Gamma}=\partial_{t}^{\bullet} \psi+\psi \nabla_{s} \cdot \vec{u}-\Delta_{s} \psi,
$$


with $\partial_{t}^{\bullet} \psi=\left(\frac{1}{2}[a(t)]^{-1} a^{\prime}(t)-6\right) \psi, \psi \nabla_{s} \cdot \vec{u}=\frac{1}{2}[a(t)]^{-1} a^{\prime}(t)\left(1-\nu_{1}^{2}\right) \psi$ and $-\Delta_{s} \psi(\vec{z}, t)=\mathrm{e}^{-6 t}\left[2 \nu_{1} \nu_{2}-\left(\nu_{1} z_{2}+\right.\right.$ $\left.\left.\nu_{2} z_{1}\right) \varkappa(\vec{z}, t)\right]$, where $\vec{\nu}(\vec{z}, t)=\frac{\nabla \phi(\vec{z}, t)}{\mid \nabla \phi(\vec{z}, t \mid} \in \mathbb{R}^{d}$ denotes the normal to $\Gamma(t)$ at $\vec{z} \in \Gamma(t)$, and where

$$
\varkappa=-\nabla_{s} \cdot \vec{\nu}=-\nabla \cdot \vec{\nu}=-|\nabla \phi|^{-1} \sum_{i=1}^{d}\left[\left(1-|\nabla \phi|^{-2}\left(\frac{\partial \phi}{\partial z_{i}}\right)^{2}\right) \frac{\partial^{2} \phi}{\partial z_{i}^{2}}\right]
$$

denotes the mean curvature of $\Gamma(t)$. Of course, for our example we have that $\nabla \phi(\vec{z}, t)=2[a(t)]^{-1} z_{1} \vec{e}_{1}+$ $2 \sum_{i=2}^{d} z_{i} \vec{e}_{i}$, and so (A.4) reduces to

$$
\varkappa=-2|\nabla \phi|^{-1}[a(t)]^{-1}\left(1-4|\nabla \phi|^{-2}[a(t)]^{-2} z_{1}^{2}\right)-2|\nabla \phi|^{-1} \sum_{i=2}^{d}\left(1-4|\nabla \phi|^{-2} z_{i}^{2}\right) .
$$

\section{REFERENCES}

[1] A. Alke and D. Bothe, 3D numerical modeling of soluble surfactant at fluidic interfaces based on the volume-of-fluid method. FDMP Fluid Dyn. Mater. Process. 5 (2009) 345-372.

[2] E. Bänsch, Finite element discretization of the Navier-Stokes equations with a free capillary surface. Numer. Math. 88 (2001) 203-235.

[3] J.W. Barrett, H. Garcke and R. Nürnberg, A stable parametric finite element discretization of two-phase Navier-Stokes flow. J. Sci. Comput. To appear in DOI: 10.1007/s10915-014-9885-2.

[4] J.W. Barrett and R. Nürnberg, Convergence of a finite-element approximation of surfactant spreading on a thin film in the presence of van der Waals forces. IMA J. Numer. Anal. 24 (2004) 323-363.

[5] J.W. Barrett, H. Garcke and R. Nürnberg, Finite element approximation of surfactant spreading on a thin film. SIAM J. Numer. Anal. 41 (2003) 1427-1464.

[6] J.W. Barrett, H. Garcke and R. Nürnberg, A parametric finite element method for fourth order geometric evolution equations. J. Comput. Phys. 222 (2007) 441-462.

[7] J.W. Barrett, H. Garcke and R. Nürnberg, On the parametric finite element approximation of evolving hypersurfaces in $\mathbb{R}^{3}$. J. Comput. Phys. 227 (2008) 4281-4307.

[8] J.W. Barrett, H. Garcke and R. Nürnberg, On stable parametric finite element methods for the Stefan problem and the Mullins-Sekerka problem with applications to dendritic growth. J. Comput. Phys. 229 (2010) 6270-6299.

[9] J.W. Barrett, H. Garcke and R. Nürnberg, Eliminating spurious velocities with a stable approximation of viscous incompressible two-phase Stokes flow. Comput. Methods Appl. Mech. Engrg. 267 (2013) 511-530.

[10] J.W. Barrett, H. Garcke and R. Nürnberg, Finite element approximation of one-sided Stefan problems with anisotropic, approximately crystalline, Gibbs-Thomson law. Adv. Differ. Eq. 18 (2013) 383-432.

[11] K. Bäumler and E. Bänsch, A subspace projection method for the implementation of interface conditions in a single-drop flow problem. J. Comput. Phys. 252 (2013) 438-457.

[12] D. Bothe, M. Köhne and J. Prüss, On two-phase flows with soluble surfactant. Preprint (2012). Available at http://arxiv . org/abs/1210.8131.

[13] D. Bothe and J. Prüss, Stability of equilibria for two-phase flows with soluble surfactant. Quart. J. Mech. Appl. Math. 63 (2010) 177-199.

[14] D. Bothe, J. Prüss and G. Simonett, Well-posedness of a two-phase flow with soluble surfactant. In Nonlinear elliptic and parabolic problems. In vol. 64 of Progr. Nonlinear Differ. Eq. Appl. (2005) 37-61.

[15] R. Clift, J.R. Grace and M.E. Weber, Bubbles, drops, and particles. Dover Publishing, Mineola, N.Y. (2005).

[16] K. Deckelnick, G. Dziuk and C.M. Elliott, Computation of geometric partial differential equations and mean curvature flow. Acta Numer. 14 (2005) 139-232.

[17] M.A. Drumright-Clarke and Y. Renardy, The effect of insoluble surfactant at dilute concentration on drop breakup under shear with inertia. Phys. Fluids 16 (2004) 14-21.

[18] G. Dziuk, An algorithm for evolutionary surfaces. Numer. Math. 58 (1991) 603-611.

[19] G. Dziuk and C.M. Elliott, Finite elements on evolving surfaces. IMA J. Numer. Anal. 27 (2007) $262-292$.

[20] G. Dziuk and C.M. Elliott, Finite element methods for surface PDEs. Acta Numer. 22 (2013) $289-396$.

[21] C.M. Elliott, B. Stinner, V. Styles and R. Welford, Numerical computation of advection and diffusion on evolving diffuse interfaces. IMA J. Numer. Anal. 31 (2011) 786-812.

[22] C.M. Elliott and V. Styles, An ALE ESFEM for solving PDEs on evolving surfaces. Milan J. Math. 80 (2012) $469-501$.

[23] S. Engblom, M. Do-Quang, G. Amberg and A.-K. Tornberg, On diffuse interface modeling and simulation of surfactants in two-phase fluid flow. Commun. Comput. Phys. 14 (2013) 879-915.

[24] S. Ganesan and L. Tobiska, A coupled arbitrary Lagrangian-Eulerian and Lagrangian method for computation of free surface flows with insoluble surfactants. J. Comput. Phys. 228 (2009) 2859-2873. 
[25] H. Garcke, K.F. Lam and B. Stinner, Diffuse interface modelling of soluble surfactants in two-phase flow. Commun. Math. Sci. 12 (2014) 1475-1522.

[26] H. Garcke and S. Wieland, Surfactant spreading on thin viscous films: nonnegative solutions of a coupled degenerate system. SIAM J. Math. Anal. 37 (2006) 2025-2048.

[27] V. Girault and P.-A. Raviart, Finite Element Methods for Navier-Stokes. Springer-Verlag, Berlin (1986).

[28] S. Groß and A. Reusken, Numerical methods for two-phase incompressible flows. Vol. 40 of Springer Ser. Comput. Math. Springer-Verlag, Berlin (2011).

[29] S. Hysing, S. Turek, D. Kuzmin, N. Parolini, E. Burman, S. Ganesan and L. Tobiska, Quantitative benchmark computations of two-dimensional bubble dynamics. Internat. J. Numer. Methods Fluids 60 (2009) 1259-1288.

[30] A.J. James and J. Lowengrub, A surfactant-conserving volume-of-fluid method for interfacial flows with insoluble surfactant. J. Comput. Phys. 201 (2004) 685-722.

[31] S. Khatri and A.-K. Tornberg, A numerical method for two phase flows with insoluble surfactants. Comput. Fluids 49 (2011) $150-165$.

[32] M.-C. Lai, Y.-H. Tseng and H. Huang, An immersed boundary method for interfacial flows with insoluble surfactant. J. Comput. Phys. 227 (2008) 7279-7293.

[33] M. Muradoglu and G. Tryggvason, A front-tracking method for computation of interfacial flows with soluble surfactants. J. Comput. Phys. 227 (2008) 2238-2262.

[34] C. Pozrikidis, A finite-element method for interfacial surfactant transport, with application to the flow-induced deformation of a viscous drop. J. Eng. Math. 49 (2004) 163-180.

[35] Y.Y. Renardy, M. Renardy and V. Cristini, A new volume-of-fluid formulation for surfactants and simulations of drop deformation under shear at a low viscosity ratio. Eur. J. Mech. B Fluids 21 (2002) 49-59.

[36] A. Schmidt and K.G. Siebert, Design of Adaptive Finite Element Software: The Finite Element Toolbox ALBERTA. Vol. 42 of Lect. Notes Comput. Sci. Eng. Springer-Verlag, Berlin (2005).

[37] K.E. Teigen, X. Li, J. Lowengrub, F. Wang and A. Voigt, A diffuse-interface approach for modeling transport, diffusion and adsorption/desorption of material quantities on a deformable interface. Commun. Math. Sci. 7 (2009) $1009-1037$.

[38] K.E. Teigen and S.T. Munkejord, Influence of surfactant on drop deformation in an electric field. Phys. Fluids 22 (2010) 112104.

[39] J.-J. Xu, Y. Huang, M.-C. Lai and Z. Li, A coupled immersed interface and level set method for three-dimensional interfacial flows with insoluble surfactant. Commun. Comput. Phys. 15 (2014) 451-469.

[40] J.-J. Xu, Z. Li, J. Lowengrub and H. Zhao, A level-set method for interfacial flows with surfactant. J. Comput. Phys. 212 (2006) 590-616.

[41] J.-J. Xu, Y. Yang and J. Lowengrub, A level-set continuum method for two-phase flows with insoluble surfactant. J. Comput. Phys. 231 (2012) 5897-5909.

[42] X. Yang and A.J. James, An arbitrary Lagrangian-Eulerian (ALE) method for interfacial flows with insoluble surfactants. FDMP Fluid Dyn. Mater. Process. 3 (2007) 65-95. 